\title{
Geometry and quasisymmetric parametrization of Semmes spaces
}

\author{
Pekka Pankka and Jang-Mei Wu
}

\begin{abstract}
We consider decomposition spaces $\mathbb{R}^{3} / G$ that are manifold factors and admit defining sequences consisting of cubes-with-handles of finite type. Metrics on $\mathbb{R}^{3} / G$ constructed via modular embeddings of $\mathbb{R}^{3} / G$ into a Euclidean space promote the controlled topology to a controlled geometry.

The quasisymmetric parametrizability of the metric space $\mathbb{R}^{3} / G \times \mathbb{R}^{m}$ by $\mathbb{R}^{3+m}$ for any $m \geq 0$ imposes quantitative topological constraints, in terms of the circulation and the growth of the cubes-with-handles, on the defining sequences for $\mathbb{R}^{3} / G$. We give a necessary condition and a sufficient condition for the existence of such a parametrization.

The necessary condition answers negatively a question of Heinonen and Semmes on quasisymmetric parametrizability of spaces associated to the Bing double. The sufficient condition gives new examples of quasispheres in $\mathbb{S}^{4}$.
\end{abstract}

\section{Introduction}

1.1. A homeomorphism $f: X \rightarrow Y$ between metric spaces $\left(X, d_{X}\right)$ and $\left(Y, d_{Y}\right)$ is called quasisymmetric if there exists a homeomorphism $\eta:[0, \infty) \rightarrow[0, \infty)$ so that

$$
\frac{d_{Y}(f(x), f(y))}{d_{Y}(f(x), f(z))} \leq \eta\left(\frac{d_{X}(x, y)}{d_{X}(x, z)}\right)
$$

for all triples $\{x, y, z\}$ in $X$. Quasisymmetry generalizes quasiconformality from Euclidean spaces to general metric spaces. A metric space $(X, d)$ is called a metric $n$-sphere if it is homeomorphic to $\mathbb{S}^{n}$.

When is a metric $n$-sphere $(X, d)$ quasisymmetrically equivalent to the standard $\mathbb{S}^{n}$ ? The goal is to find intrinsic qualitative metric properties of the space $(X, d)$ that recognize such geometric equivalence. A complete characterization of quasispheres is known only for dimensions 1 and 2 .

Mathematics Subject Classification (2010): Primary 30L10; Secondary 30L05, 30C65.

Keywords: Quasisymmetry, parametrization, quasisphere, decomposition space. 
In dimension 1, a result of Tukia and Väisälä [21] states a metric 1-sphere $(X, d)$ is quasisymmetrically equivalent to $\mathbb{S}^{1}$ if and only if $X$ is doubling and is of bounded turning. Bonk and Kleiner (Theorem 1.1 in [5]) give a characterization in dimension 2. A consequence of their theorems states that a metric 2-sphere $(X, d)$ is quasisymmetrically equivalent to $\mathbb{S}^{2}$ if $X$ is linearly locally contractible and Ahlfors 2-regular. Semmes proved this result earlier for metric spaces with some added smoothness properties (Section 5 in [17]). Wildrick proved recently an analogue of Bonk and Kleiner's result for $\mathbb{R}^{2}[23]$.

A metric space $(X, d)$ is said to be linearly locally contractible if for a fixed $C>1$ every ball of radius $r<1 / C$ is contractible in a concentric ball of radius $C r$; and $X$ is said to be Ahlfors 2-regular if there exists a measure $\mu$ on the space so that the $\mu$-measure of every ball of radius $r$ is uniformly comparable to $r^{2}$.

Could a metric space which is homeomorphic to $\mathbb{S}^{n}$ or $\mathbb{R}^{n}$, and resembles $\mathbb{S}^{n}$ or $\mathbb{R}^{n}$, geometrically (linearly locally contractible), measure-theoretically (Ahlfors $n$-regular), and analytically (supports Poincaré and Sobolev inequalities) in dimensions $n \geq 3$, fail to be quasisymmetrically equivalent to $\mathbb{S}^{n}$ or $\mathbb{R}^{n}$ ?

Semmes's counterexample [19] to this natural question in dimension 3 is a geometrically self-similar space modeled on the decomposition space $\mathbb{R}^{3} / \mathrm{Bd}$ associated to the Bing double Bd. The classical construction of R. H. Bing in geometric topology gives an example of a wild involution in $\mathbb{R}^{3}$. As a topological space $\mathbb{R}^{3} / \mathrm{Bd}$ is homeomorphic to $\mathbb{R}^{3}$.

Semmes shows that this space admits a metric that is smooth Riemannian outside a totally disconnected closed set and, in many ways, indistinguishable from the standard metric on $\mathbb{R}^{3}$, and yet the space is not quasisymmetrically equivalent to $\mathbb{R}^{3}$. In Semmes's metric the $2^{k}$ tori at $k$ th stage of the construction of $\mathbb{R}^{3} / \mathrm{Bd}$ are uniformly round and thick, whereas under any homeomorphism from $\mathbb{R}^{3} / \mathrm{Bd}$ to $\mathbb{R}^{3}$, there exists a sequence of tori that are distorted into a shape longer and thinner than allowed by any fixed quasisymmetry. Semmes's construction is robust and essentially available in all decomposition spaces of $\mathbb{R}^{3}$ arising from topologically self-similar constructions.

The natural conditions for metric $n$-spheres listed earlier are also insufficient in higher dimensions. The decomposition space $\mathbb{R}^{3} / \mathrm{Wh}$ associated to the Whitehead continuum Wh is not homeomorphic to $\mathbb{R}^{3}$, but $\mathbb{R}^{3} / \mathrm{Wh} \times \mathbb{R}$ is homeomorphic to $\mathbb{R}^{4}$. In [12], Heinonen and the second author showed that the decomposition space $\mathbb{R}^{3} /$ Wh associated to the Whitehead continuum Wh admits a linearly locally contractible and Ahlfors 3-regular metric, but $\left(\mathbb{R}^{3} / \mathrm{Wh}\right) \times \mathbb{R}^{m}$ is not quasisymmetrically equivalent to $\mathbb{R}^{3+m}$ for any $m \geq 1$. The metric on $\mathbb{R}^{3} / \mathrm{Wh}$ is due to Semmes; as in the case of $\mathbb{R}^{3} / \mathrm{Bd}$ this metric makes the tori in the construction of the Whitehead continuum uniformly round and thick.

The Whitehead link, formed by a meridian of the first torus and the core of the second torus, however prevents the conformal modulus of a sequence of surface families over longitudes of the nested tori from being quasi-preserved under any homeomorphism $\mathbb{R}^{3} / \mathrm{Wh} \times \mathbb{R}^{m} \rightarrow \mathbb{R}^{3+m}$.

1.2. The decomposition space $\mathbb{R}^{3} / \mathrm{Wh}$ is only one example of an exotic manifold factor of $\mathbb{R}^{4}$. By a theorem of Edwards and Miller [7], decomposition spaces that 


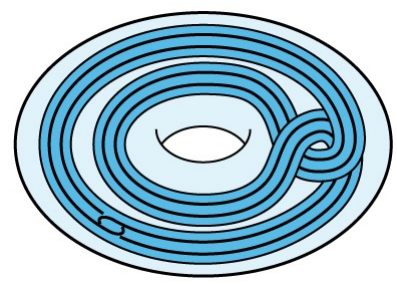

Figure 1. Two generations of Whitehead links.

are exotic factors of $\mathbb{R}^{4}$ exist in abundance. In fact, cell-like closed 0-dimensional upper semicontinuous decomposition spaces $\mathbb{R}^{3} / G$ are manifold factors of $\mathbb{R}^{4}$, that is, $\mathbb{R}^{3} / G \times \mathbb{R}$ is homeomorphic to $\mathbb{R}^{4}$. Furthermore, under mild assumptions on the decomposition, these spaces are definable by nested sequences $\mathcal{X}=\left(X_{k}\right)_{k \geq 0}$ of unions of cubes-with-handles, i.e., the degenerate part of the decomposition $G$ is $\bigcap_{k>0} X_{k}$; see Lambert and Sher [14] and Sher and Alford [20]. This class of decomposition spaces provides a natural environment for testing the quasisymmetric parametrization.

In this article, we consider a subclass of decomposition spaces $\mathbb{R}^{3} / G$ that are manifold factors and admit defining sequences of finite type. The corresponding defining sequences $\mathcal{X}=\left(X_{k}\right)_{k \geq 0}$ satisfy the a priori condition

$$
\#\left\{\left[H \backslash \operatorname{int} X_{k+1}\right]_{\mathrm{PL}}: k \geq 0 \text { and } H \text { is a component of } X_{k}\right\}<\infty \text {; }
$$

here $[E]_{\mathrm{PL}}$ is the PL-homeomorphism equivalence class of a PL-manifold $E \subset \mathbb{R}^{3}$.

The definition of finite type is based on the notion of welding. A welding structure $(\mathcal{C}, \mathcal{A}, \mathcal{W})$ consists of condensers $\mathcal{C}$, an atlas $\mathcal{A}$ composed of charts, and weldings $\mathcal{W}$ determined by the atlas $\mathcal{A}$. The condensers can be seen as fixed geometric realizations of PL-homeomorphism equivalence classes of components of differences $X_{k} \backslash \operatorname{int} X_{k+1}$ in the defining sequence $\mathcal{X}$, and the charts in the atlas $\mathcal{A}$ determine the parametrization of these components. The weldings, in turn, are transition maps between the charts; see Section 4.

Definition 1.1. A defining sequence $\mathcal{X}=\left(X_{k}\right)_{k \geq 0}$ has finite type if there exists a welding structure $(\mathcal{C}, \mathcal{A}, \mathcal{W})$ with finitely many condensers $\mathcal{C}$ and finitely many weldings $\mathcal{W}$. A decomposition space $\left(\mathbb{R}^{3} / G, \mathcal{X}\right)$ is of finite type if the defining sequence $\mathcal{X}$ has finite type.

We take up a systematic study of the geometric realizations which promote the controlled topology to a controlled geometry. Using results from classical geometric topology, we construct for every defining sequence of finite type a geometrically simple welding structure, called a rigid welding structure, having translations as weldings; see Theorem 5.2.

A rigid welding structure allows the natural geometrization of the decomposition space $\mathbb{R}^{3} / G$. Given a rigid welding structure $(\mathcal{C}, \mathcal{A}, \mathcal{W})$ and a scaling factor $\lambda \in(0,1)$, we show that there exists a modular embedding of $\mathbb{R}^{3} / G$ into a Euclidean 

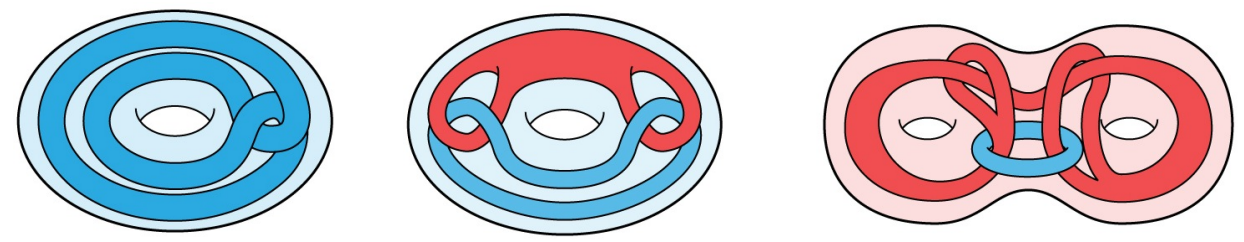

FiguRE 2. These condensers generate an infinite number of defining sequences of finite type.

space that respects the atlas $\mathcal{A}$ and the chosen scaling factor $\lambda$; see Theorem 6.2 . The metric $d_{\lambda}$ induced on $\mathbb{R}^{3} / G$ by a modular embedding is called a Semmes metric and the corresponding metric space a Semmes space; these metrics naturally extend the class of metrics constructed by Semmes in [19].

For a fixed rigid welding structure, the Semmes spaces $\left(\mathbb{R}^{3} / G, d_{\lambda}\right)$ for all scalings are mutually quasisymmetric. We find it appealing that, although $\mathbb{R}^{3} / G$ does not admit a canonical metric, there exists a natural class of metrics on $\mathbb{R}^{3} / G$ respecting the defining sequence $\mathcal{X}$ whose quasisymmetry equivalence classes are parametrized by rigid welding structures on $\mathcal{X}$ modulo compatible atlases; see Proposition 7.10 .

We summarize the essential features of this geometrization process of decomposition spaces by Semmes metrics in the following theorem; see Sections 6 and 7 for these results. Given a Semmes metric $d_{\lambda}$ on $\mathbb{R}^{3} / G$, we equip the space $\mathbb{R}^{3} / G \times \mathbb{R}^{m}$ with the product metric $d_{\lambda, m}((x, v),(y, w))=d_{\lambda}(x, y)+|v-w|$.

Theorem 1.2. Let $\left(\mathbb{R}^{3} / G, \mathcal{X}\right)$ be a decomposition space of finite type. Then there exists a Semmes metric $d_{\lambda}$ on $\mathbb{R}^{3} / G$ so that, for each $m \geq 0,\left(\mathbb{R}^{3} / G \times \mathbb{R}^{m}, d_{\lambda, m}\right)$ is a quasiconvex Ahlfors $(3+m)$-regular Loewner space that admits an isometric embedding into a Euclidean space. Moreover, the space $\left(\mathbb{R}^{3} / G \times \mathbb{R}^{m}, d_{\lambda, m}\right)$ is linearly locally contractible if the sequence $\mathcal{X}$ is locally contractible.

A defining sequence $\mathcal{X}=\left(X_{k}\right)_{k \geq 0}$ is locally contractible if every component of $X_{k+1}$ is contractible in $X_{k}$ for all $k \geq 0$. We emphasize that the linear local contractibility and the Loewner property are necessary for the existence of a quasisymmetric parametrization; see Semmes [19], Heinonen and Koskela [9], and Tyson [22].

1.3. Having this general theory at our disposal, we now discuss the problem of quasisymmetric parametrization.

Due to the quasi-invariance of the conformal modulus, the existence of a quasisymmetric homeomorphism between $\mathbb{R}^{3} / G \times \mathbb{R}^{m}$ and $\mathbb{R}^{3+m}$ imposes a relation between geometry (growth of the handlebodies and the fixed scaling factor) and topology (circulation of the handlebodies).

The order of growth of $\mathcal{X}$ controls the growth of the number of components of $X_{k}$ in the sequence as $k$ tends to infinity; see Definition 4.2. The order of 
circulation of $\mathcal{X}$ reflects the growth of the unsigned linking numbers of the longitudes of handlebodies of $X_{k^{\prime}}$ with respect to the meridians of $X_{k}$, for $k^{\prime}>k$; see Definition 9.2.

Theorem 1.3. Let $\mathbb{R}^{3} / G$ be a decomposition space of finite type associated to a locally contractible defining sequence $\mathcal{X}$. Suppose that the order of growth of the defining sequence $\mathcal{X}$ is at most $\gamma$, the order of circulation is at least $\omega$, where $\gamma, \omega \in[0, \infty]$, and

$$
\omega^{3}>\gamma^{2}
$$

Then there exists a Semmes metric on $\mathbb{R}^{3} / G$ so that $\mathbb{R}^{3} / G \times \mathbb{R}^{m}$ is a linearly locally contractible, Ahlfors $(3+m)$-regular, Loewner space but not quasisymmetrically equivalent to $\mathbb{R}^{3+m}$ for any $m \geq 0$.

For the Whitehead continuum and the Bing double we may take the pair $(\gamma, \omega)$ to be $(1,2)$ and $(2,2)$, respectively. In particular, Theorem 1.3 provides a negative answer to a question of Heinonen and Semmes in [11] (Question 11). The case $m=0$ in the following theorem is Semmes's quasisymmetric non-parametrizability result of $\mathbb{R}^{3} / \mathrm{Bd}$ in [19].

Theorem 1.4. The decomposition space $\mathbb{R}^{3} / \mathrm{Bd}$ associated to the Bing double admits a metric that is Ahlfors 3-regular, Loewner, and linearly locally contractible but none of the spaces $\mathbb{R}^{3} / \mathrm{Bd} \times \mathbb{R}^{m}$ for $m \geq 0$ is quasisymmetrically equivalent to $\mathbb{R}^{3+m}$.

Theorem 1.3 admits a local formulation as stated in Theorem 14.3. This local version examines inequality (1.1) on a branch of the defining sequence at a point in $\mathbb{R}^{3} / G$; it is generally more applicable. Whereas inequality (1.1) gives a natural necessary condition for a quasisymmetric parametrization of $\left(\mathbb{R}^{3} / G \times \mathbb{R}^{m}, d_{\lambda, m}\right)$ by $\mathbb{R}^{3+m}$, the pointwise inequality (14.1) provides a criterion for the quasisymmetric equivalence of the product spaces $\left(\mathbb{R}^{3} / G \times \mathbb{R}, d_{\lambda, 1}\right)$ for $0<\lambda<1$. This yields, for example, the following inequivalence result for the product spaces associated to the Bing double.

Theorem 1.5. Let $\left(\mathbb{R}^{3} / \mathrm{Bd}, d_{\lambda}\right)$ be a Semmes space associated to the Bing double. For any $\lambda^{\prime} \in(1 / 2,1)$ and $\lambda \in\left(0, \lambda^{\prime}\right)$, the spaces $\left(\mathbb{R}^{3} / \mathrm{Bd} \times \mathbb{R}, d_{\lambda, 1}\right)$ and $\left(\mathbb{R}^{3} / \mathrm{Bd} \times\right.$ $\left.\mathbb{R}, d_{\lambda^{\prime}, 1}\right)$ are quasisymmetrically inequivalent.

When applying Theorem 1.3 and Theorem 14.3, estimating the order of circulation from below for a particular decomposition space can be a challenging topological problem of its own. For decomposition spaces associated to the Bing double [19], to the Whitehead continuum [12], or to Bing's dogbone (Section 18), the circulation is estimated by adapting a theorem of Freedman and Skora [8] on counting essential intersections by relative homologies; see Section 17.

1.4. Theorem 1.3 imposes a topological condition on the parametrization. In the opposite direction, additional Euclidean restrictions on the welding structure yield 
positive results about the quasisymmetric parametrization of $\mathbb{R}^{3} / G$ by $\mathbb{R}^{3}$. These geometric assumptions on the defining sequence are encapsulated in the notion of flat welding structure; see Section 8.

Theorem 1.6. Let $\mathbb{R}^{3} / G$ be a decomposition space of finite type that admits a defining sequence with a flat welding structure in $\mathbb{R}^{3}$. Then there exists a linearly locally contractible, Ahlfors 3 -regular metric on $\mathbb{R}^{3} / G$ so that $\mathbb{R}^{3} / G$ is quasisymmetric to $\mathbb{R}^{3}$. Moreover, there exist an isometric embedding $\theta: \mathbb{R}^{3} / G \rightarrow \mathbb{R}^{4}$ and a quasisymmetric homeomorphism $f: \mathbb{R}^{4} \rightarrow \mathbb{R}^{4}$ so that $f\left(\mathbb{R}^{3}\right)=\theta\left(\mathbb{R}^{3} / G\right)$.

These decomposition spaces give new examples of quasispheres in $\mathbb{R}^{4}$ as formulated in the second part of the theorem.

In light of Theorem 1.6, we ask about the sharpness of the condition (1.1) in Theorem 1.3, especially for a fixed $m$. In case of $\mathbb{R}^{3}$ (i.e., $m=0$ ) the construction of Antoine's necklaces $G$ using $I$ linked tori yields decomposition spaces with order of growth $I$ and order of circulation at least 2. Semmes's result on the Bing double implies that the decomposition space $\left(\mathbb{R}^{3} / G, d\right)$ associated with a necklace constructed using two linked tori, when equipped with a Semmes metric $d$, is not quasisymmetric to $\mathbb{R}^{3}$.

The existence of a quasisymmetric parametrization of $\mathbb{R}^{3} / G$ when $I$ is sufficiently large has been observed by Heinonen and Rickman [10] using similar round tori. Using rectangular tori in place of round tori, we prove in Theorem 16.1 that for every $I \geq 10$, the decomposition space $\mathbb{R}^{3} / G$ associated to Antoine's I-necklace may be equipped with a Semmes metric so that it is quasisymmetrically equivalent to $\mathbb{R}^{3}$.

Having these examples at hand, the real test for the sharpness of Theorem 1.3 seems to be the quasisymmetric parametrizability of the decomposition space associated to the Antoine's 3-necklace.

Acknowledgments. Authors are grateful to the anonymous referee for his thoughtful comments, which led to the clarification of some mathematical concepts and the improvement in the presentation. Authors are indebted to Julie Kaufman for providing the schematic drawings in this article.

Both authors would like to thank Kevin Wildrick for his continuing interest in this work. The first author thanks Eero Saksman for discussions, and the Department of Mathematics at the University of Illinois at Urbana-Champaign for hospitality during his numerous visits.

\section{Contents}

1 Introduction . . . . . . . . . . . . . . . . . . . 893

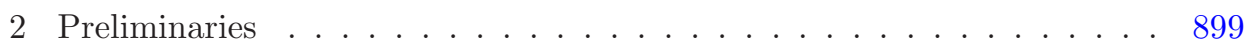

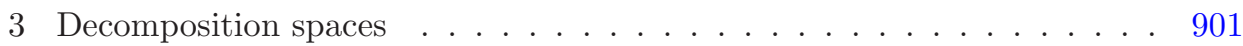

4 Welding structures . . . . . . . . . . . . . . . . . . . 904

5 Rigid welding structures . . . . . . . . . . . . . . . . . 907

6 Modular embeddings . . . . . . . . . . . . . . . . . . 910 
7 Semmes spaces . . . . . . . . . . . . . . . . . . . . . 917

8 A sufficient condition for quasisymmetric parametrization . . . . . . . 924

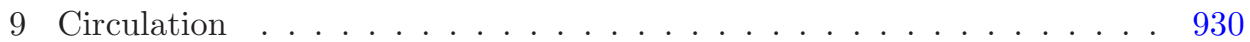

10 Circulation and a modulus estimate for walls $\ldots \ldots \ldots \ldots \ldots 35$

11 Quasisymmetric tubes . . . . . . . . . . . . . . . . . . . 939

12 Growth and a modulus estimate for walls . . . . . . . . . . . . 941

13 A necessary condition for quasisymmetric parametrization . . . . . . . 945

14 Local parametrizability . . . . . . . . . . . . . . . . . . . . . 946

15 Singular fibers of Semmes spaces _ . . . . . . . . . . . . . . 947

16 Necklaces . . . . . . . . . . . . . . . . . . . . . . 950

17 The Bing double and the Whitehead continuum revisited . . . . . . 955

18 Bing's dogbone . . . . . . . . . . . . . . . . . . . . . 956

\section{Preliminaries}

Unless otherwise stated, we assume that $\mathbb{R}^{n}, n \geq 1$, is equipped with the Euclidean metric and the standard basis $\left(e_{1}, \ldots, e_{n}\right)$. We denote by $B^{n}(x, r)$ the closed Euclidean ball in $\mathbb{R}^{n}$ of radius $r$ and center $x$. For brevity, the closed balls centered on the origin are denoted $B^{n}(r)=B^{n}(0, r)$ for $r>0$ and $\mathbb{B}^{n}=B^{n}(1)$. Similarly, $S^{n-1}(x, r)=\partial B^{n}(x, r)$ is the Euclidean sphere of radius $r$ and center $x$ in $\mathbb{R}^{n}$, and $S^{n-1}(r)=S^{n-1}(0, r)$ for $r>0$ and $\mathbb{S}^{n-1}=S^{n-1}(1)$.

For all $1 \leq m<n$, we identify $\mathbb{R}^{m}$ with the subspace $\mathbb{R}^{m} \times\{0\}$ in $\mathbb{R}^{n}$ where $\{0\}$ is the origin in $\mathbb{R}^{n-m}$, and identify a set $A \subset \mathbb{R}^{m}$ with the set $A \times\{0\}$ in $\mathbb{R}^{m} \times \mathbb{R}^{n-m}$. When $\mathbb{R}^{n}$ is expressed as $\mathbb{R}^{m} \times \mathbb{R}^{p} \times \mathbb{R}^{q}$ with $m, p, q>0, n=m+p+q$, a subset of $\mathbb{R}^{n}$ in the form $A \times B \times C$ is understood to have the property that $A \subset \mathbb{R}^{m}, B \subset \mathbb{R}^{p}$, and $C \subset \mathbb{R}^{q}$.

By a map, we always mean a continuous map. Given a map $F: X \times[0,1] \rightarrow Y$, we denote by $F_{t}: X \rightarrow Y$ the map $F_{t}(x)=F(x, t)$. We say that a homotopy $F: X \times[0,1] \rightarrow Y$ is an isotopy if $F_{t}$ is a homeomorphism for all $t \in[0,1]$.

We call a map $\alpha: I \rightarrow X$ from an interval in $\mathbb{R}$ into a metric space $X$ a path and maps $\mathbb{S}^{1} \rightarrow X$ loops. If there is no confusion we do not distinguish between a map and its image. Images of paths and loops are also called curves. A loop $\mathbb{S}^{1} \rightarrow X$ is simple if it is an embedding.

Given a set $E$ in a metric space $(X, d)$ and a number $a>0$, we call

$$
N_{d}(E, a)=\left\{x \in X: \operatorname{dist}_{d}(x, E)<a\right\}
$$

the $a$-neighborhood of $E$ in $X$. When $X=\mathbb{R}^{n}$ and $d$ is the Euclidean metric, we write $N^{n}(E, a)$ for $N_{d}(E, a)$. We denote by $\mathcal{C}(E)$ the set of all connected components of $E$.

Given a metric space $(X, d)$ so that points in the space can be connected by rectifiable paths, we denote by $\hat{d}$ the path metric of $(X, d)$ defined by

$$
\hat{d}(x, y)=\inf _{\gamma} \ell_{d}(\gamma)
$$


for $x, y \in X$, where $\ell_{d}(\gamma)$ is the length of path $\gamma$ in the metric $d$ and the infimum is taken over all paths $\gamma$ connecting $x$ and $y$ in $X$. A metric space $(X, d)$ is called quasiconvex if id: $(X, \hat{d}) \rightarrow(X, d)$ is bilipschitz.

A metric space $(X, d)$ is Ahlfors $Q$-regular if there exist a Borel measure $\mu$ in $X$ and a constant $C \geq 1$ so that

$$
\frac{1}{C} r^{Q} \leq \mu(B(x, r)) \leq C r^{Q}
$$

for every ball $B(x, r)$ of radius $r \leq \operatorname{diam} X$ centered on $x$ in $X$. Furthermore, the space $(X, d)$ is locally linearly contractible if there exists $C \geq 1$ so that the ball $B(x, r)$ in $X$ is contractible in $B(x, C r)$ for all $r<1 / C$.

We say that a mapping $f:\left(X, d_{X}\right) \rightarrow\left(Y, d_{Y}\right)$ between metric spaces is a $(\lambda, L)$ quasisimilarity if

$$
\frac{\lambda}{L} d_{X}(x, y) \leq d_{Y}(f(x), f(y)) \leq \lambda L d_{X}(x, y)
$$

for all $x, y \in X$. Clearly, quasisimilarities are a subclass of quasisymmetries. As usual, we call $(\lambda, 1)$-quasisimilarities similarities and 1-similarities isometries. The $(1, L)$-quasisimilarities are $L$-bilipschitz mappings. In what follows, we abuse notation and write $|x-y|=d(x, y)$ when there is no ambiguity as to what is the metric in question.

In what follows, we consider only Lipschitz chains of multiplicity one, that is, we consider only $m$-chains $\sigma$ so that $\sigma=\sum_{i=1}^{k} \sigma_{i}$, where $\sigma_{i}:[0,1]^{m} \rightarrow X$ is a Lipschitz map for $i=1, \ldots, k$. In a metric measure space $(X, d, \mu)$ we define the p-modulus of an $m$-chain family as follows.

Given a family $\Sigma$ of $m$-chains in a $X$, the $p$-modulus of $\Sigma$ is

$$
\operatorname{Mod}_{p}(\Sigma)=\inf _{\rho} \int_{X} \rho^{p} \mathrm{~d} \mu,
$$

where $\rho$ is a nonnegative Borel function satisfying

$$
\sum_{i=1}^{k} \int_{\sigma_{i}\left([0,1]^{m}\right)} \rho \mathrm{d} \mathcal{H}^{m} \geq 1
$$

for all $\sigma=\sum_{i=1}^{k} \sigma_{i} \in \Sigma$.

In what follows, handlebodies are three dimensional piecewise linear cubeswith-handles embedded in $\mathbb{R}^{n}$. For this we assume in what follows that $\mathbb{R}^{n}$ is given a fixed PL-structure for every $n \geq 3$.

We use the following topological facts on cubes-with-handles; see Chapter 2 of [13] for more details. We say that $H$ is a cube-with-handles if it is a regular neighborhood of an embedded rose $\iota\left(\bigvee^{g} \mathbb{S}^{1}\right)$, where $\iota: \bigvee^{g} \mathbb{S}^{1} \rightarrow \mathbb{R}^{3}$ is a PL-embedding. Here $\bigvee^{g} \mathbb{S}^{1}$ is the wedge of $g$ circles, that is, the identification of $g$ circles at a point; $\bigvee^{0} \mathbb{S}^{1}$ is a point. The number $g$ of circles in the rose is called the number of handles of $H$ or the genus of $H$. The image $\iota\left(\bigvee^{g} \mathbb{S}^{1}\right)$ is called a core of $H$. 
The genus of $H$ is also the maximal number of essentially embedded 2-disks whose union separates $H$. We say that a disk $D$ in $H$ is essentially embedded if there exists an embedding $\varphi:\left(\mathbb{B}^{2}, \partial \mathbb{B}^{2}\right) \rightarrow(H, \partial H)$ so that $\varphi \mid \partial \mathbb{B}^{2}: \partial \mathbb{B}^{2} \rightarrow \partial H$ is not null-homotopic in $\partial H$.

The genus of $H$ is a topological invariant. Two cube-with-handles $H$ and $H^{\prime}$ in $\mathbb{R}^{3}$ are PL homeomorphic if and only if they have the same number of handles and both are either orientable or nonorientable (Theorem 2.2 in [13]). We denote by $\mathrm{g}(H)$ the genus of $H$.

A three-dimensional cube-with-handles in $\mathbb{R}^{n}$ need not be orientable for $n>3$, but a three-dimensional cube-with-handles in $\mathbb{R}^{3}$ inherits an orientation from $\mathbb{R}^{3}$ and is therefore orientable.

\section{Decomposition spaces}

We begin this section by reviewing some classical results on decomposition spaces relevant to our study. We do not aim at the full generality and refer to Daverman [6] for details.

A decomposition $G$ of a topological space $X$ is a partition of $X$. Associated with $G$ is the decomposition space $X / G$ equipped with the topology induced by the quotient map $\pi_{G}: X \rightarrow X / G$, the richest topology for which $\pi_{G}$ is continuous, see $[6]$, p. 8 .

A decomposition $G$ is upper semicontinuous (usc) if each $g \in G$ is closed and if for every $g \in G$ and every neighborhood $U$ of $g$ in $X$ there exists a neighborhood $V$ of $g$ contained in $U$ so that every $g^{\prime} \in G$ intersecting $V$ is contained in $U$. If $G$ is usc then $X / G$ is metrizable (Definition I.2 and Proposition I.2.2 in [6]); however there is not a canonical metric on $X / G$.

Suppose that $G$ is a usc decomposition of an $n$-manifold $M$ and $d$ is a metric on $M / G$. The decomposition map $\pi_{G}: M \rightarrow M / G$ can be approximated by homeomorphisms if and only if $G$ satisfies Bing's shrinkability criterion (Theorem II.5.2 in [6]). In particular, $M / G$ is homeomorphic to $M$.

Bing's shrinkability criterion states that for every $\varepsilon>0$ there is a homeomorphism $h: M \rightarrow M$ such that

1. $\operatorname{diam} h(g)<\varepsilon$ for each $g \in G$, and

2. $d\left(\pi_{G} h(x), \pi_{G}(x)\right)<\varepsilon$ for every $x \in M$.

Suppose $M$ is an $n$-manifold. If $G$ is a shrinkable usc decomposition then each $g \in G$ is cellular, therefore cell-like (see Proposition II.6.1 and Corollary III.15.2B in [6]). A subset $Z$ of $M$ is cellular if for each open $U \supset Z$ there is an $n$-cell $E$ such that $Z \subset$ int $E \subset E \subset U$; recall that an $n$-cell is a subset homeomorphic to $\mathbb{B}^{n}$. A compact set $Z$ in a space $X$ is cell-like in $X$ if $Z$ can be contracted to a point in every neighborhood of $Z$.

Certain decomposition spaces can be constructed from defining sequences. A defining sequence for a decomposition of an $n$-manifold $M$ is a sequence $\mathcal{X}=\left(X_{k}\right)_{k \geq 0}$ of compact sets satisfying int $X_{k} \supset X_{k+1}$. The decomposition $G$ associated to the 
defining sequence $\mathcal{X}$ consists of the components of $X_{\infty}=\bigcap_{k>0} X_{k}$ and the singletons from $M \backslash X_{\infty}$, see [6], p. 61. The decomposition $G$ associated to $\mathcal{X}$ is upper semicontinuous and $\pi_{G}\left(X_{\infty}\right)$ is compact and 0-dimensional, see Proposition II.9.1 in $[6]$.

In the context of defining sequences, a sufficient condition for $\mathbb{R}^{3} / G$ to be homeomorphic with $\mathbb{R}^{3}$ is the following shrinking criterion: For each $k \geq 1$ and each $\epsilon>0$, there exist $\ell \geq 1$ and a homeomorphism $h$ of $\mathbb{R}^{3}$ onto itself satisfying $h \mid\left(\mathbb{R}^{3} \backslash X_{k}\right)=\mathrm{id}$, and $\operatorname{diam} h(H)<\epsilon$ for all components $H$ of $X_{k+\ell}$.

Convention. In what follows, all decomposition spaces $\mathbb{R}^{3} / G$ are derived from defining sequences $\mathcal{X}$ consisting of (unions of) cubes-with-handles. At times, we denote the space by $\left(\mathbb{R}^{3} / G, \mathcal{X}\right)$ to emphasize the role of the sequence $\mathcal{X}$.

We fix some notation for use in later sections. Let $\mathcal{X}=\left(X_{k}\right)_{k \geq 0}$ be a defining sequence. We denote by $\mathcal{C}(\mathcal{X})=\bigcup_{k} \mathcal{C}\left(X_{k}\right)$ all components of the defining sequence $\mathcal{X}=\left(X_{k}\right)_{k \geq 0}$; here $\mathcal{C}(E)$ denotes the set of components of the set $E$.

Given $H \in \mathcal{C}(\mathcal{X})$ there is a unique index $k \geq 0$ so that $H \in \mathcal{C}\left(X_{k}\right)$. We call the index $k$ the level of $H$ and write level $(H)=k$. For every $H \in \mathcal{C}(\mathcal{X})$, we write

$$
H^{\text {diff }}=H \backslash \operatorname{int} X_{\text {level }(H)+1} .
$$

Then $\mathcal{C}\left(H \backslash \operatorname{int} H^{\text {diff }}\right)$ consists of all components of $X_{\text {level }(H)+1}$ contained in $H$.

Given two cubes-with-handles $H$ and $H^{\prime}$ in $\mathcal{C}(\mathcal{X})$, we have

$$
H=H^{\prime}, H^{\prime} \subset \operatorname{int} H, H \subset \operatorname{int} H^{\prime}, \text { or } H \cap H^{\prime}=\emptyset \text {. }
$$

Thus $\partial H \cap X_{\infty}=\emptyset$ for every $H \in \mathcal{C}(\mathcal{X})$. Since $X_{\infty}$ is closed in $\mathbb{R}^{3}$, there exists, for every $H \in \mathcal{C}(\mathcal{X})$, a neighborhood $\Omega_{\partial H}$ of $\partial H$ in $\mathbb{R}^{3}$ so that $\pi_{G} \mid \Omega_{\partial H}$ is an embedding.

At times we shall write $\mathbb{R}^{3} / X_{\infty}$ for $\mathbb{R}^{3} / G$ for simplicity, in particular when $X_{\infty}$ is a Whitehead continuum, a necklace, a Bing double, or a Bing's dogbone.

\subsection{Decomposition spaces as manifold factors}

Our main interest lies in decomposition spaces $\mathbb{R}^{3} / G$ that are homeomorphic to $\mathbb{R}^{3}$ or whose product, $\mathbb{R}^{3} / G \times \mathbb{R}^{m}$, with a Euclidean space is homeomorphic to $\mathbb{R}^{3+m}$ for some $m>0$. Decomposition spaces of the latter type are called manifold factors of Euclidean spaces.

By results of Sher and Alford and Lambert and Sher (Theorem 1 in [20], and [14]), if $G$ is a cell-like usc decomposition of $\mathbb{R}^{3}$ so that the closure of all nondegenerate elements of $G$ is 0 -dimensional, then $G$ admits a defining sequence consisting of (unions of) cubes-with-handles. Subsequently Edwards and Miller (see [7], p. 192) proved that if $G$ satisfies the conditions of Lambert and Sher, then $\mathbb{R}^{3} / G$ is a factor of $\mathbb{R}^{4}$, that is,

$$
\mathbb{R}^{3} / G \times \mathbb{R} \approx \mathbb{R}^{4},
$$


and $G \times \mathbb{R}$ is a shrinkable decomposition of $\mathbb{R}^{4}$, see also Section V.27 in [6]. In particular, the quotient map $\pi^{\prime}: \mathbb{R}^{3+m} \rightarrow \mathbb{R}^{3+m} /\left(G \times \mathbb{R}^{m}\right)$ can be approximated by homeomorphisms. The composition

$$
\left(\pi_{G} \times \mathrm{id}\right) \circ\left(\pi^{\prime}\right)^{-1}: \mathbb{R}^{3+m} /\left(G \times \mathbb{R}^{m}\right) \rightarrow \mathbb{R}^{3} / G \times \mathbb{R}^{m}
$$

is a homeomorphism ([6], Proposition I.2.4). Therefore

$$
\mathbb{R}^{3} / G \times \mathbb{R}^{m} \approx \mathbb{R}^{3+m}
$$

and $\pi_{G} \times$ id: $\mathbb{R}^{3+m} \rightarrow \mathbb{R}^{3} / G \times \mathbb{R}^{m}$ can be approximated by homeomorphisms.

Let $\mathbb{R}^{3} / G$ be a decomposition space associated to a locally contractible defining sequence $\mathcal{X}=\left(X_{k}\right)_{k \geq 0}$ consisting of unions of cubes-with-handles. That is, every component of $X_{k+1}$ is contractible in $X_{k}$, for all $k \geq 0$. Then, by Edwards-Miller, $\mathbb{R}^{3} / G \times \mathbb{R}$ is homeomorphic to $\mathbb{R}^{4}$. Indeed, under this assumption on $\mathcal{X}$, the components of $X_{\infty}$ are cell-like and $\pi_{G}\left(X_{\infty}\right)$ is compact and 0-dimensional.

\subsection{Local contractibility}

In this section, we establish a local contractibility property for $\pi_{G}\left(X_{k}\right)$ in the decomposition space $\mathbb{R}^{3} / G$ from the local contractiblity of a defining sequence $\mathcal{X}=\left(X_{k}\right)_{k \geq 0}$.

Lemma 3.1. Let $\mathbb{R}^{3} / G$ be a decomposition space associated to a locally contractible defining sequence $\mathcal{X}=\left(X_{k}\right)_{k \geq 0}$. Then components of $\pi_{G}\left(X_{k+1}\right)$ are contractible in $\pi_{G}\left(X_{k}\right)$ for $k \geq 0$.

Lemma 3.1 follows directly from the following cellularity property of the decomposition $G \times \mathbb{R}^{m}$ for $m \geq 1$.

Lemma 3.2. Let $m \geq 1$ and let $\mathbb{R}^{3} / G$ be a decomposition space associated to a locally contractible defining sequence $\mathcal{X}=\left(X_{k}\right)_{k \geq 0}$. Then, for every $k \geq 0$, $H^{\prime} \in \mathcal{C}\left(X_{k}\right), H \in \mathcal{C}\left(X_{k+1} \cap H^{\prime}\right)$, and $r>0$, there exists a $(3+m)$-cell $E$ so that

$$
\pi_{G}(H) \times[-r, r]^{m} \subset E \subset \pi_{G}\left(H^{\prime}\right) \times(-2 r, 2 r)^{m} .
$$

Proof of Lemma 3.1. Let $k \geq 0, H^{\prime} \in \mathcal{C}\left(X_{k}\right)$, and $H \in \mathcal{C}\left(X_{k+1} \cap H^{\prime}\right)$. To show that $\pi_{G}(H)$ is contractible in $\pi_{G}\left(H^{\prime}\right)$, let $m=1$ and $r>0$, and let $E$ be a 4-cell in $\mathbb{R}^{3} / G \times \mathbb{R}$ as in Lemma 3.2.

Denote by proj the projection $\mathbb{R}^{3} / G \times \mathbb{R} \rightarrow \mathbb{R}^{3} / G$. We identify $\mathbb{R}^{3} / G$ with $\mathbb{R}^{3} / G \times\{0\}$ in $\mathbb{R}^{3} / G \times \mathbb{R}$. Since $E$ is an 4-cell, $\pi_{G}(H)$ is contractible in $E$. Thus $\pi_{G}(H)$ is contractible in $\operatorname{proj}(E) \subset \pi_{G}\left(H^{\prime}\right)$.

The proof of Lemma 3.2 is based on an approximation of the quotient map $\pi_{G} \times \mathrm{id}: \mathbb{R}^{3+m} \rightarrow \mathbb{R}^{3} / G \times \mathbb{R}^{m}$ by homeomorphisms, and the classical PenroseWhitehead-Zeeman lemma (Lemma 2.7 in [15]): Let $M$ be an $n$-manifold and let $P \subset \operatorname{int} M$ be an $(q-1)$-dimensional polyhedron $(1 \leq q \leq n / 2)$ such that the inclusion map $i: P \rightarrow M$ is homotopic in $M$ to a constant. Then there exists an $n$-cell $E \subset \operatorname{int} M$ such that $P \subset \operatorname{int} E$. 
Proof of Lemma 3.2. Let $r>0$ and $k \geq 0$, and let $H^{\prime} \in \mathcal{C}\left(X_{k}\right)$ and $H \in \mathcal{C}\left(X_{k+1} \cap\right.$ $H^{\prime}$ ) be cubes-with-handles in $\mathcal{X}$. Let $\delta$ be any metric on the decomposition space $\mathbb{R}^{3} / G$, and let $\delta_{m}$ be the product of $\delta$ with the Euclidean metric on $\mathbb{R}^{3} / G \times \mathbb{R}^{m}$. Let $a_{0}=\min \left\{r, \operatorname{dist}_{\delta}\left(\partial \pi_{G}(H), \partial \pi_{G}\left(H^{\prime}\right)\right)\right\}$.

We fix cores $\mathcal{R}$ and $\mathcal{R}^{\prime}$ of $H$ and $H^{\prime}$, respectively. Then $H$ and $H^{\prime}$ are regular neighborhoods of $\mathcal{R}$ and $\mathcal{R}^{\prime}$, respectively. By adding a one-sided collar of the boundary $\partial H$ to $H$, we obtain a regular neighborhood $H^{\prime \prime}$ of $\mathcal{R}$ containing $H$ in the interior. Similarly, by removing a one-sided collar of $\partial H^{\prime}$ in $H^{\prime}$, we obtain a regular neighborhood $H^{\prime \prime \prime}$ of $\mathcal{R}$ contained in $H^{\prime}$; see Corollaries 2.26 and 3.17 in [16]. Moreover, we require that

$$
H \subset \operatorname{int} H^{\prime \prime} \subset H^{\prime \prime} \subset \operatorname{int} H^{\prime \prime \prime} \subset H^{\prime \prime \prime} \subset \operatorname{int} H^{\prime} \subset H^{\prime},
$$

and that $a_{0} / 10<\operatorname{dist}_{\delta}\left(x, \partial \pi_{G} H\right)<a_{0} / 9$ for all $x \in \partial \pi_{G} H^{\prime \prime}$ and $a_{0} / 10<$ $\operatorname{dist}_{\delta}\left(x, \partial \pi_{G} H^{\prime}\right)<a_{0} / 9$ for all $x \in \partial \pi_{G} H^{\prime \prime \prime}$.

Since $H$ is contractible in $H^{\prime}$, we have that $H^{\prime \prime}$ is contractible in $H^{\prime \prime \prime}$. By the Penrose-Whitehead-Zeeman lemma, there exists a $(3+m)$-cell $E^{\prime}$ so that

$$
\mathcal{R} \times\{0\} \subset H^{\prime \prime} \times\left(-\frac{5}{4} r, \frac{5}{4} r\right)^{m} \subset E^{\prime} \subset H^{\prime \prime \prime} \times\left(-\frac{3}{2} r, \frac{3}{2} r\right)^{m} .
$$

Since $\pi_{G} \times$ id can be approximated by homeomorphisms, by the Edwards-Miller theorem, we may fix a homeomorphism $h: \mathbb{R}^{3+m} \rightarrow \mathbb{R}^{3} / G \times \mathbb{R}^{m}$ so that

$$
\max _{(x, v) \in X_{0} \times[-3 r, 3 r]^{m}} \delta_{m}\left(h(x, v),\left(\pi_{G}(x), v\right)\right)<a_{0} / 100 .
$$

Then $h^{-1}\left(\pi_{G} H \times[-r, r]^{m}\right) \subset H^{\prime \prime} \times\left(-\frac{5}{4} r, \frac{5}{4} r\right)^{m}$ and $h\left(H^{\prime \prime \prime} \times\left(-\frac{3}{2} r, \frac{3}{2} r\right)^{m}\right) \subset$ $\pi_{G} H^{\prime} \times(-2 r, 2 r)^{m}$. Thus $E=h\left(E^{\prime}\right)$ is a $(3+m)$-cell satisfying

$$
\pi_{G}(H) \times[-r, r]^{m} \subset E \subset \pi_{G}\left(H^{\prime}\right) \times(-2 r, 2 r)^{m} .
$$

\section{Welding structures}

Let $n \geq 3$. By abusing the standard terminology in potential theory, we say that a pair $(A, B)$ is a condenser in $\mathbb{R}^{n}$ if $A$ is a 3 -dimensional cube-with-handles in $\mathbb{R}^{n}$ and $B$ is a disjoint union of 3 -dimensional cubes-with-handles in $\mathbb{R}^{n}$ so that $B \subset \operatorname{int} A$; here $\operatorname{int} A$ is the manifold interior of $A$. Given a condenser $\mathrm{c}=(A, B)$, we set

$$
\mathrm{c}^{\text {diff }}=A \backslash \operatorname{int} B \text {. }
$$

Given two condensers $\mathrm{c}=(A, B)$ and $\mathrm{c}^{\prime}=\left(A^{\prime}, B^{\prime}\right)$ in $\mathbb{R}^{n}$, a PL-embedding $\psi: \partial A^{\prime} \rightarrow \partial B$ is said to be a welding of $\mathrm{c}^{\prime}$ to $\mathrm{c}$. Since $\partial A^{\prime}$ is a closed surface and $\partial B$ is a disjoint union of closed surfaces in $\mathbb{R}^{n}, \psi\left(\partial A^{\prime}\right)$ is a component of $\partial B$. Here $\partial M$ is the two dimensional manifold boundary of a 3-manifold $M$.

Let $\mathcal{X}$ be a defining sequence and $\mathcal{C}$ a family of condensers in $\mathbb{R}^{n}$. Suppose that for each $H \in \mathcal{C}(\mathcal{X})$, there exist a condenser $\mathrm{c}_{H}=\left(A_{H}, B_{H}\right) \in \mathcal{C}$ and a PL-homeomorphism $\varphi_{H}: H^{\text {diff }} \rightarrow c_{H}^{\text {diff }}$ satisfying $\varphi_{H}(\partial H)=\partial A_{H}$ and $\varphi_{H}\left(\partial H^{\text {diff }} \backslash \partial H\right)$ $=\partial B_{H}$. Then we call

$$
\mathcal{A}=\left\{\varphi_{H}\right\}_{H \in \mathcal{C}(\mathcal{X})}
$$

an atlas for $\mathcal{X}$, and the elements of $\mathcal{A}$ charts. 
Let $\mathcal{C}$ be a family of condensers and $\mathcal{A}=\left\{\varphi_{H}\right\}_{H \in \mathcal{C}(\mathcal{X})}$ an atlas for $\mathcal{X}$. Given $H \in$ $\mathcal{C}(\mathcal{X})$ and $H^{\prime} \in \mathcal{C}\left(H \cap X_{\text {level }(H)+1}\right)$, let $\mathrm{c}_{H}=\left(A_{H}, B_{H}\right)$ and $\mathrm{c}_{H^{\prime}}=\left(A_{H^{\prime}}, B_{H^{\prime}}\right)$ be the corresponding condensers in $\mathcal{C}$. We define the induced welding $\psi_{H, H^{\prime}}: \partial A_{H}^{\prime} \rightarrow \partial B_{H}$ by the formula

$$
\psi_{H, H^{\prime}}=\varphi_{H} \circ \varphi_{H^{\prime}}^{-1} \mid \partial A_{H^{\prime}} .
$$

We denote the induced welding scheme by

$$
\mathcal{W}=\left\{\psi_{H, H^{\prime}}: \partial A_{H^{\prime}} \rightarrow \partial B_{H}\right\}_{\left(H, H^{\prime}\right)},
$$

where $H \in \mathcal{C}(\mathcal{X})$ and $H^{\prime} \in \mathcal{C}\left(H \cap X_{\text {level }(H)+1}\right)$;

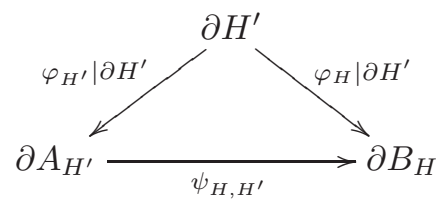

The triple $(\mathcal{C}, \mathcal{A}, \mathcal{W})$ is called a welding structure on $\mathcal{X}$.

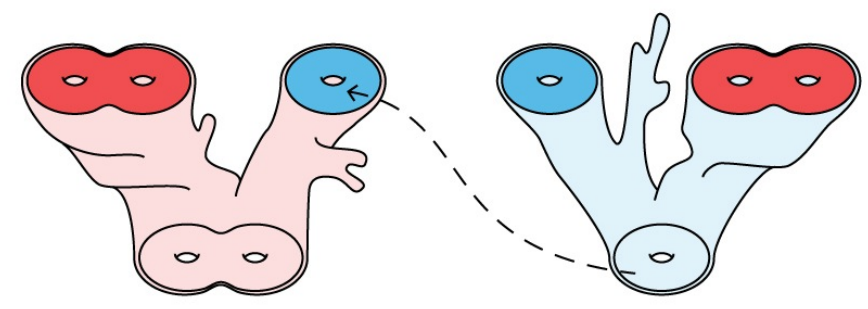

Figure 3. A welding between two condensers.

\subsection{Defining sequences of finite type}

Recall from the introduction that a defining sequence $\left(X_{k}\right)_{k>0}$ is of finite type if there exists a welding structure $(\mathcal{C}, \mathcal{A}, \mathcal{W})$ with $\# \mathcal{C}<\infty$ and $\# \mathcal{W}<\infty$. A decomposition space $\left(\mathbb{R}^{3} / G, \mathcal{X}\right)$ is of finite type if $\mathcal{X}$ has finite type.

The definition of a welding structure allows condensers to lie in high dimensional Euclidean spaces. However, a welding structure in $\mathbb{R}^{3}$ can always be built from the original defining sequence.

Proposition 4.1. Let $\mathcal{X}$ be a defining sequence of finite type. Then there exists a welding structure $(\mathcal{C}, \mathcal{A}, \mathcal{W})$ in $\mathbb{R}^{3}$ so that

(i) for each $\mathrm{c}=(A, B) \in \mathcal{C}$, there exists $H \in \mathcal{C}(\mathcal{X})$ for which $(A, B)=(H, H \cap$ $X_{\text {level }(H)+1)}$; and

(ii) $\# \mathrm{C}<\infty$ and $\# \mathcal{W}<\infty$. 
Proof. Let $\left(\mathcal{C}^{\prime}, \mathcal{A}^{\prime}, \mathcal{W}^{\prime}\right)$ be a welding structure for $\mathcal{X}$ so that $\# \mathcal{C}^{\prime}<\infty$ and $\# \mathcal{W}^{\prime}<\infty$. We may assume that each condenser $\mathrm{c}=(A, B) \in \mathrm{C}^{\prime}$ is the image of a chart, that is, there exists $H \in \mathcal{C}(\mathcal{X})$ for which $\mathrm{c}_{H}=\mathrm{c}$ and $\varphi_{H}:\left(H^{\text {diff }}, \partial H\right) \rightarrow\left(\mathrm{c}_{H}^{\text {diff }}, \partial A_{H}\right)$. We fix for each $c \in \mathcal{C}^{\prime}$ such a cube-with-handles and denote it by $H_{\mathrm{c}}$. Let $\phi_{\mathrm{c}}: \mathrm{c}^{\text {diff }} \rightarrow H_{\mathrm{c}}^{\text {diff }}$ be the inverse of the chart $\varphi_{H_{\mathrm{c}}}$.

Define

$$
\mathcal{C}=\left\{\left(H_{\mathrm{c}}, H_{\mathrm{c}} \cap X_{\text {level }\left(H_{\mathrm{c}}\right)+1}\right\}_{\mathrm{c} \in \mathcal{C}^{\prime}}\right.
$$

and

$$
\mathcal{A}=\left\{\phi_{\mathrm{c}_{H}} \circ \varphi_{H}\right\}_{H \in \mathcal{C}(\mathcal{X})},
$$

Since $\mathcal{W}^{\prime}$ is a finite collection, the charts in $\mathcal{A}$ induce a finite collection weldings $\mathcal{W}$ between boundary components of condensers in $\mathcal{C}$. Thus $(\mathcal{C}, \mathcal{A}, \mathcal{W})$ satisfies the conditions of the claim.

Let $\mathcal{X}=\left(X_{k}\right)_{k \geq 0}$ be a defining sequence of finite type. Then the cubes-withhandles in $\mathcal{C}(\mathcal{X})$ have uniformly bounded genus; we define

$$
\overline{\mathrm{g}}_{\mathcal{X}}=\max \{\mathrm{g}(H): H \in \mathcal{C}(\mathcal{X})\} .
$$

Furthermore, $\mathcal{X}$ has a finite (upper) growth

$$
\bar{\gamma}_{\mathcal{X}}=\max \left\{\# \mathcal{C}\left(X_{k+1} \cap H\right): H \in \mathcal{C}\left(X_{k}\right), k \geq 0\right\} .
$$

Definition 4.2. The order of growth $\gamma_{\mathcal{X}}$ of $\mathcal{X}$ is defined to be

$$
\gamma_{\mathcal{X}}=\lim _{r \rightarrow \infty} \max \left\{\# \mathcal{C}\left(X_{k+1} \cap H\right): H \in \mathcal{C}\left(X_{k}\right), k \geq r\right\}
$$

\subsection{Self-similar spaces}

Self-similar decomposition spaces are examples of decomposition spaces of finite type. Semmes's initial packages for defining self-similar decomposition spaces yield almost directly finite welding structures on the defining sequences if the initial packages are understood in the PL-category instead of the smooth category; see Section 3 of [19].

An initial package $\left(T, T_{1}, \ldots, T_{N}, \phi_{1}, \ldots, \phi_{N}\right)$ consists of cubes-with-handles $T, T_{1}, \ldots, T_{N}$ in $\mathbb{R}^{3}$ with $T_{i} \subset \operatorname{int} T$ and $T_{i} \cap T_{i^{\prime}}=\emptyset$ for $i \neq i^{\prime}$, together with PL-embeddings $\phi_{i}: U \rightarrow T$ of a neighborhood $U$ of $T$ into $T$ so that $\phi_{i}(T)=T_{i}$ and the images $\phi_{i}(U)$ are mutually disjoint neighborhoods of $T_{i}$ 's. The defining sequence $\mathcal{X}=\left(X_{k}\right)_{k \geq 0}$ is given by $X_{0}=T$ and

$$
X_{k}=\bigcup_{\alpha} \phi_{\alpha}(T)
$$

for $k \geq 1$, where $\alpha=\left(\alpha_{1}, \ldots, \alpha_{k}\right) \in\{1, \ldots, N\}^{k}$ and $\phi_{\alpha}=\phi_{\alpha_{1}} \circ \cdots \circ \phi_{\alpha_{k}}$.

Let $\mathrm{c}=\left(T, \bigcup_{i=1}^{N} \phi_{i}(T)\right)$ be a condenser. Then homeomorphisms $\left(\phi_{\alpha} \mid \mathrm{c}^{\text {diff }}\right)^{-1}$ : $\phi_{\alpha}(T)^{\text {diff }} \rightarrow \mathrm{c}^{\text {diff }}, \alpha \in \bigcup_{k \geq 0}\{1, \ldots, N\}^{k}$, form an atlas $\mathcal{A}$ for $\mathcal{X}$. Although $\mathcal{A}$ is an infinite atlas, the associated collection of weldings

$$
\mathcal{W}=\left\{\phi_{i} \mid \partial T: 1 \leq i \leq N\right\}
$$


is finite. We call $(\{\mathrm{c}\}, \mathcal{A}, \mathcal{W})$ the welding structure associated to the initial package $\left(T, T_{1}, \ldots, T_{N}, \phi_{1}, \ldots, \phi_{N}\right)$.
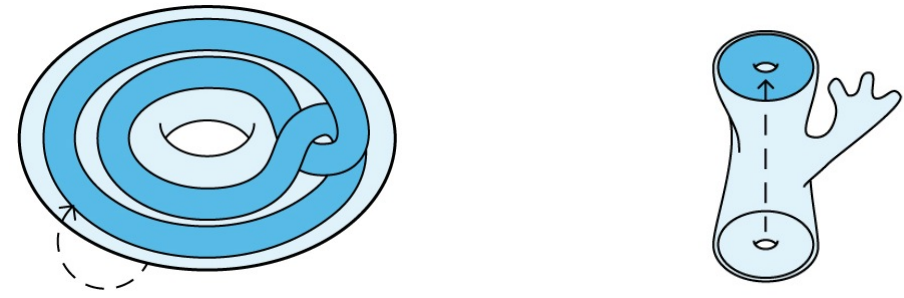

Figure 4. Two welding structures associated to the Whitehead construction in Figure 1.

We refer to Section 3 of [19] for more details on initial packages for self-similar decomposition spaces.

\section{Rigid welding structures}

We introduce now rigid welding structures which correspond to the excellent packages of Semmes ([19], Definition 3.2). In our terminology, Semmes's excellent packages translate to welding structures with one condenser in $\mathbb{R}^{4}$, whose boundary lies entirely in $\mathbb{R}^{3} \times\{0\}$, and with similarities as weldings. Semmes showed the existence of excellent packages for defining sequences associated to the Whitehead continuum, Bing's dogbone, and the Bing double; see Sections 4-6 of [19].

Definition 5.1. Let $(\mathcal{C}, \mathcal{A}, \mathcal{W})$ be a welding structure on a defining sequence $\mathcal{X}$ of finite type. We call $(\mathcal{C}, \mathcal{A}, \mathcal{W})$ a rigid welding structure in $\mathbb{R}^{n}, n \geq 4$, if $\mathcal{C}$ consists of finitely many condensers and

(S1) all boundary components of differences $\left\{c^{\text {diff }}: c \in \mathcal{C}\right\}$ of the same genus are translations of one another,

(S2) weldings in $\mathcal{W}$ are translations,

(S3) for every $\mathrm{c}=(A, B) \in \mathcal{C}$ we have that $\partial A \subset \mathbb{B}^{3} \times\{0\} \subset \mathbb{R}^{4} \subset \mathbb{R}^{n}, B \subset$ $\mathbb{B}^{3} \times\{1\} \subset \mathbb{R}^{4} \subset \mathbb{R}^{n}$, and int $\left(\mathrm{c}^{\text {diff }}\right) \subset \mathbb{B}^{3} \times(0,1) \times \mathbb{R}^{n-4}$.

For self-similar defining sequences, the existence of an excellent package induces a natural embedding of the space $\mathbb{R}^{3} / G$ into $\mathbb{R}^{4}$ (see Lemma 3.21 in [19]). The possibility to place $B \cup \partial A$ on two separate levels and to use all dimensions $n \geq 4$ is less restrictive than the requirements for excellent packages. For this reason, all defining sequence of finite type admit rigid welding structures. Whereas Semmes's excellent packages lie in $\mathbb{R}^{4}$, the rigid welding structures lie in a fixed space $\mathbb{R}^{16}$. As the dimension of the ambient space containing condensers does not play a significant role in the construction of metrics, we make no attempt to obtain the optimal ambient dimension for rigid welding structures. 
Theorem 5.2 (Existence of rigid welding structures). Let $\left(\mathbb{R}^{3} / G, \mathcal{X}\right)$ be a decomposition space of finite type. Then $\mathcal{X}$ admits a rigid welding structure in $\mathbb{R}^{16}$.

To straighten the condensers and the weldings between condensers, we apply the Klee trick; see Proposition II.10.4 in [6].

Lemma 5.3. Let $m \geq 1$ and $k \geq 1$, and let $E$ be a $P L$ compact set in $\mathbb{R}^{k}$, and let $f: E \rightarrow \mathbb{R}^{m}$ be a PL-embedding. Then there exists a PL-homeomorphism $h: \mathbb{R}^{k+m} \rightarrow \mathbb{R}^{k+m}$ so that $h \mid E \times\{0\}=f$.

To obtain condensers satisfying (S3), we use the following lemma based on general position.

Lemma 5.4. Suppose $\mathrm{c}=(A, B)$ is a condenser in $\mathbb{R}^{n}, n \geq 8$, so that $\partial A \subset$ $\mathbb{B}^{3} \times\{0\} \subset \mathbb{R}^{4} \subset \mathbb{R}^{n}$ and $B \subset \mathbb{B}^{3} \times\{1\} \subset \mathbb{R}^{4} \subset \mathbb{R}^{n}$. Then there exists a $P L$ embedding $F: A \rightarrow \mathbb{R}^{n}$ so that $F \mid \partial A \cup B=$ id and $F\left(c^{\text {diff }}\right) \subset \mathbb{B}^{3} \times(0,1) \times \mathbb{R}^{n-4}$.

Proof. We fix $t>1$ and $t^{\prime}<0$ so that $A \subset \mathbb{R}^{3} \times\left(t^{\prime}, t\right) \times \mathbb{R}^{n-4}$.

Since $\partial B \times[1, t]$ is a 3 -dimensional PL-manifold in $\mathbb{R}^{4} \subset \mathbb{R}^{n}$ and $c^{\text {diff }}$ is 3 -dimensional, there exists, by general position (see Theorem 5.3 in [16]), a PL-homeomorphism $h: \mathbb{R}^{3} \times \mathbb{R} \times \mathbb{R}^{n-4} \rightarrow \mathbb{R}^{3} \times \mathbb{R} \times \mathbb{R}^{n-4}$ satisfying $h \mid \mathbb{R}^{3} \times(\mathbb{R} \backslash(1, t)) \times \mathbb{R}^{n-4}=\mathrm{id}$ and $h(\partial B \times(1, t]) \cap \mathrm{c}^{\text {diff }}=\emptyset$.

Let $B^{\prime}=B+t e_{4}, A^{\prime}=(A \backslash B) \cup h(\partial B \times[1, t]) \cup B^{\prime}$, and $c^{\prime}=\left(A^{\prime}, B^{\prime}\right)$. Since $h(\partial B \times[1, t])$ is a one-sided collar of $\partial B$, there exists a PL-homeomorphism $k: A \rightarrow A^{\prime}$ so that $k \mid \partial A=\mathrm{id}$ and $k \mid B$ is the translation $(x, 1, y) \mapsto(x, t, y)$, where $x \in \mathbb{R}^{3}$ and $y \in \mathbb{R}^{n-4}$.

Let $g: \mathbb{R}^{3} \times \mathbb{R} \times \mathbb{R}^{n-4} \rightarrow \mathbb{R}^{3} \times \mathbb{R} \times \mathbb{R}^{n-4}$ be the map $(x, s, y) \mapsto(x, s / t, y)$. Then $\mathrm{c}^{\prime \prime}=g\left(\mathrm{c}^{\prime}\right)=\left(A^{\prime \prime}, B^{\prime \prime}\right)$ is a condenser so that $\left(\mathrm{c}^{\prime \prime}\right)^{\text {diff }} \subset \mathbb{R}^{3} \times(-\infty, 1) \times \mathbb{R}^{n-4}$. Note that $g \circ k \mid(\partial A \cup B)=$ id. Since the same argument can be applied to $\left[t^{\prime}, 0\right]$, we may assume that int $\left(\mathrm{c}^{\prime \prime}\right)^{\text {diff }} \subset \mathbb{R}^{3} \times(0,1) \times \mathbb{R}^{n-4}$.

We fix a piecewise linear function $\nu: \mathbb{R} \rightarrow(0,1)$ and a PL-homeomorphism $f: \mathbb{R}^{3} \times \mathbb{R} \times \mathbb{R}^{n-4} \rightarrow \mathbb{R}^{3} \times \mathbb{R} \times \mathbb{R}^{n-4}, f(x, s, y)=(\nu(s) x, s, y)$, so that $\nu(s)=1$ for $s \notin(0,1)$ and $f\left(\left(c^{\prime \prime}\right)\right.$ diff $\left.\cap \mathbb{R}^{3} \times\{s\}\right) \subset \mathbb{B}^{3} \times\{s\}$ for $s \in(0,1)$. Since $f \mid \partial A \cup B=\mathrm{id}$, the composition $F=f \circ g \circ k$ satisfies the requirements of the claim.

Proof of Theorem 5.2. Let $(\mathcal{C}, \mathcal{A}, \mathcal{W})$ be the welding structure in $\mathbb{R}^{3}$ associated to $\mathcal{X}$ as in Proposition 4.1. As a preliminary step, we fix, for every $0 \leq g \leq \bar{g}_{\mathcal{X}}$, a cubewith-handles $T_{g}$ of genus $g$ in $\mathbb{R}^{3}$.

Step 1: We straighten the boundary components of the condensers.

Let $\mathrm{c}=(A, B)$ be a condenser in $\mathcal{C}$. We fix a point $z_{D} \in \mathbb{R}^{3} \times\{1\}$ for each component $D \in \mathcal{C}(B)$ so that the cubes-with-handles $T_{g_{D}}+z_{D}$ are pairwise disjoint, where $g_{D}$ is the genus of $D$. Fix also a regular neighborhood $E$ of $\partial A$ in $\mathbb{R}^{3}$ so that $E \cap B=\emptyset$ and an embedding $f: E \cup B \rightarrow \mathbb{R}^{3} \times\{0,1\}$ such that $f(\partial A)=$ $\partial T_{g_{A}} \subset \mathbb{R}^{3} \times\{0\}$ and $f(D)=T_{g_{D}}+z_{D} \subset \mathbb{R}^{3} \times\{1\}$ for each $D \in \mathcal{C}(B)$. Then, by Lemma 5.3, there exists a PL-homeomorphism $h_{\mathrm{c}}: \mathbb{R}^{8} \rightarrow \mathbb{R}^{8}$ such that $h_{\mathrm{c}}(\partial A)=$ $\partial T_{g_{A}} \subset \mathbb{R}^{3} \times\{0\}$ and $h_{\mathrm{c}}(D)=T_{g_{D}}+z_{D}$ for every $D \in \mathcal{C}(B)$. 
Homeomorphisms $h_{\mathrm{c}}$ induce a new welding structure with the condensers $\tilde{\mathrm{C}}=$ $\left\{\left(h_{\mathrm{c}}(A), h_{\mathrm{c}}(B)\right): \mathrm{c} \in \mathcal{C}\right\}$, the atlas $\tilde{\mathcal{A}}=\left\{h_{\mathrm{c}_{H}} \circ \varphi_{H}: H \in \mathcal{C}(\mathcal{X})\right\}$, and the weldings $\tilde{\mathcal{W}}$ defined by $\tilde{\mathcal{C}}$ and $\tilde{\mathcal{A}}$.

We denote the new structure $(\tilde{\complement}, \tilde{\mathcal{A}}, \tilde{\mathcal{W}})$ in $\mathbb{R}^{8}$ again by $(\mathcal{C}, \mathcal{A}, \mathcal{W})$, and the new condensers, charts, and weldings again by c, $\varphi_{H}$, and $\psi_{H, H^{\prime}}=\varphi_{H} \circ \varphi_{H^{\prime}}^{-1}$ respectively.

Step 2: We now straighten the weldings from Step 1 to translations while expanding the collection of condensers.

Let $\psi: \partial A_{1} \rightarrow \partial B_{2}$ be a welding in $\mathcal{W}$ between the condensers $\left(A_{1}, B_{1}\right)$ and $\left(A_{2}, B_{2}\right)$ in $\mathcal{C}$. Let $D \in \mathcal{C}\left(B_{2}\right)$ be the component receiving $\psi$, that is, $\psi\left(\partial A_{1}\right)=\partial D$. Since $D$ and $A_{1}$ have the same genus, we may fix a translation $\tau_{\psi}: \partial A_{1} \rightarrow \partial D$. Set

$$
\hat{\mathcal{W}}=\left\{\tau_{\psi}\right\}_{\psi \in \mathcal{W}}
$$

We will add to $\mathcal{C}$ a new condenser for each welding in $\hat{\mathcal{W}}$ and modify the existing charts in $\mathcal{A}$. This new atlas has $\hat{\mathcal{W}}$ as the collection of induced weldings.

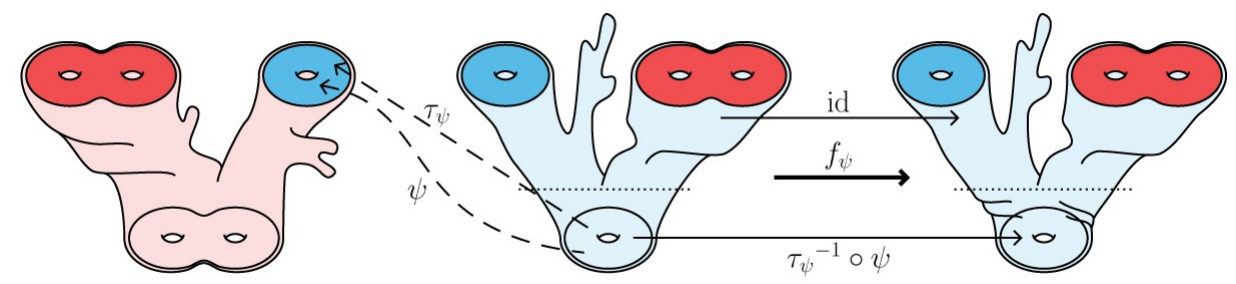

FiguRE 5. The construction of a new condenser, $\mathrm{c}_{\psi}$.

We first define the new condensers. Let $\psi: \partial A_{1} \rightarrow \partial B_{2}$ be a welding in $\mathcal{W}$ between the condensers $\left(A_{1}, B_{1}\right)$ and $\left(A_{2}, B_{2}\right)$ in $\mathcal{C}$. Fix a one-sided collar $E$ of $\partial A_{1}$ in $A_{1} \backslash B_{1}$ and an open set $U \subset \mathbb{R}^{8}$ satisfying $A_{1} \cap U \subset E$. By the Klee trick (Lemma 5.3), there exists a homeomorphism $f_{\psi}: \mathbb{R}^{16} \rightarrow \mathbb{R}^{16}$ so that $f_{\psi} \mid A_{1} \backslash U=$ id and $f_{\psi} \mid \partial A_{1}=\tau_{\psi}^{-1} \circ \psi$. We set $\mathrm{c}_{\psi}=\left(f_{\psi}\left(A_{1}\right), B_{1}\right)$, and note that $B_{1} \subset A_{1} \backslash U$ and thus $f_{\psi} \mid B_{1}=$ id. We define

$$
\hat{\mathrm{C}}=\mathcal{C} \cup\left\{\mathbf{c}_{\psi}: \psi \in \mathcal{W}\right\}
$$

Since $\# \mathfrak{C}+\# \mathcal{W}<\infty, \hat{\mathfrak{C}}$ is a finite collection of condensers satisfying (S1).

We finish the proof by defining the atlas $\hat{\mathcal{A}}$. For $H^{\prime} \in \mathcal{C}(\mathcal{X})$ with level $\left(H^{\prime}\right)=0$, we define $\hat{c}_{H^{\prime}}=\mathrm{c}_{H^{\prime}}$ and $\hat{\varphi}_{H^{\prime}}=\varphi_{H^{\prime}}$. Suppose now that $H^{\prime} \in \mathcal{C}(\mathcal{X})$ has level at least 1 and let $H \in \mathcal{C}(\mathcal{X})$ be the cube-with-handles satisfying $H^{\prime} \in \mathcal{C}\left(H \cap X_{\text {level }(H)+1}\right)$. Let $\varphi_{H}: H^{\text {diff }} \rightarrow \mathrm{c}_{H}$ and $\varphi_{H^{\prime}}: H^{\prime \text { diff }} \rightarrow \mathrm{c}_{H^{\prime}}$ be the corresponding charts in $\mathcal{A}$, and $\psi=\psi_{H, H^{\prime}}$ the welding induced by $\varphi_{H}$ and $\varphi_{H^{\prime}}$. We define $\hat{c}_{H^{\prime}}=\mathrm{c}_{\psi}$ and set $\hat{\varphi}_{H^{\prime}}: H^{\text {diff }} \rightarrow \mathrm{c}_{\psi}^{\text {diff }}$ by the formula $f_{\psi} \circ \varphi_{H^{\prime}}$. Define

$$
\hat{\mathcal{A}}=\left\{\hat{\varphi}_{H^{\prime}}\right\}_{H^{\prime} \in \mathcal{C}(\mathcal{X})} \text {. }
$$


To check that weldings induced by charts in $\hat{\mathcal{A}}$ are in $\hat{\mathcal{W}}$, let $H \in \mathcal{C}(\mathcal{X})$ and $H^{\prime} \in$ $\mathcal{C}\left(H \cap X_{\text {level }(H)+1}\right)$ be as above, and define $\hat{c}_{H^{\prime}}=\left(\hat{A}_{H^{\prime}}, \hat{B}_{H^{\prime}}\right)$. Since $\hat{\varphi}_{H} \mid \partial H^{\prime}=$ $\varphi_{H} \mid \partial H^{\prime}$, we have

$$
\hat{\varphi}_{H} \circ \hat{\varphi}_{H^{\prime}}^{-1}\left|\partial \hat{A}_{H^{\prime}}=\varphi_{H} \circ \varphi_{H^{\prime}}^{-1} \circ f_{\psi_{H, H^{\prime}}}^{-1}\right| \partial \hat{A}_{H^{\prime}}=\psi_{H, H^{\prime}} \circ f_{\psi_{H, H^{\prime}}}^{-1} \mid \partial \hat{A}_{H^{\prime}}=\tau_{\psi_{H, H^{\prime}}} .
$$

Thus weldings are in $\hat{\mathcal{W}}$ and satisfy (S2).

Step 3: To obtain condensers satisfying condition (S3) we first apply a translation and a scaling in $\mathbb{R}^{3}$ (with the same scaling constant) to all condensers in $\hat{\mathcal{C}}$ so that the assumptions of Lemma 5.4 are satisfied. Then we apply Lemma 5.4 and change the atlas accordingly.

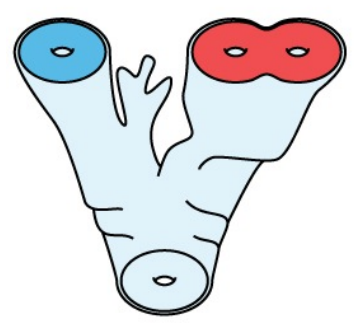

FIgURE 6. A condenser in a rigid structure.

\section{Modular embeddings}

In this section we discuss embeddings of decomposition spaces of finite type into Euclidean spaces. Given a welding structure, we first introduce the notion of a modular embedding of $\mathbb{R}^{3} / G$ into a Euclidean space, which respects the quasisimilarity type of that structure. This embedding defines a geometrically natural modular metric on the decomposition space $\mathbb{R}^{3} / G$.

It has been shown in the previous section that a defining sequence of finite type admits a rigid welding structure. Theorem 6.2 proves the existence of a modular embedding with respect to any rigid structure.

Given a welding structure $(\mathcal{C}, \mathcal{A}, \mathcal{W})$ on a decomposition space $\left(\mathbb{R}^{3} / G, \mathcal{X}\right)$ of finite type with $0<\lambda<1$ and $n \geq 3$, we say that an embedding $\theta: \mathbb{R}^{3} / G \rightarrow \mathbb{R}^{n}$ is $\lambda$-modular (with respect to $(\mathcal{C}, \mathcal{A}, \mathcal{W}))$ if $\theta \circ \pi_{G} \mid\left(\mathbb{R}^{3} \backslash X_{0}\right)=$ id and there exists $L \geq 1$ so that

$$
\theta \circ \pi_{G} \circ \varphi_{H}^{-1}: \mathrm{c}_{H}^{\mathrm{diff}} \rightarrow \mathbb{R}^{n}
$$

is a $\left(\lambda^{k}, L\right)$-quasisimilarity for every $H \in \mathcal{C}\left(X_{k}\right)$ and $k \geq 0$, that is, the following 
diagram commutes:

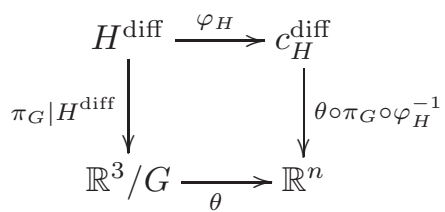

Given a $\lambda$-modular embedding $\theta: \mathbb{R}^{3} / G \rightarrow \mathbb{R}^{n}$ with respect to a welding structure $(\mathcal{C}, \mathcal{A}, \mathcal{W})$, we define the $\lambda$-modular metric $d_{\theta}$ on $\mathbb{R}^{3} / G$ by

$$
d_{\theta}(x, y)=|\theta(x)-\theta(y)|
$$

here $|\cdot|$ is the Euclidean norm on $\mathbb{R}^{n}$.

We need the notion of compatible atlases to compare the modular metrics induced by modular embeddings with respect to two different welding structures. Welding structures $(\mathcal{C}, \mathcal{A}, \mathcal{W})$ and $\left(\mathcal{C}^{\prime}, \mathcal{A}^{\prime}, \mathcal{W}^{\prime}\right)$ on $\mathcal{X}$ are said to have compatible atlases if there exists $L \geq 1$ so that

$$
\varphi_{H}^{\prime} \circ \varphi_{H}^{-1} \mid c_{H}^{\text {diff }}: c_{H}^{\text {diff }} \rightarrow\left(c_{H}^{\prime}\right)^{\text {diff }}
$$

is $L$-bilipschitz for every $H \in \mathcal{C}(\mathcal{X})$, where homeomorphisms $\varphi_{H}: H^{\text {diff }} \rightarrow c_{H}^{\text {diff }}$ and $\varphi_{H}^{\prime}: H^{\text {diff }} \rightarrow\left(\mathrm{c}_{H}^{\prime}\right)^{\text {diff }}$ are charts in $\mathcal{A}$ and $\mathcal{A}^{\prime}$, respectively.

Lemma 6.1. Let $\left(\mathbb{R}^{3} / G, \mathcal{X}\right)$ be a decomposition space of finite type and let $\lambda \in$ $(0,1)$. Suppose $\left(\mathcal{C}_{i}, \mathcal{A}_{i}, \mathcal{W}_{i}\right), i=1,2$, are welding structures on $\mathcal{X}$ having compatible atlases, and let $\theta_{i}: \mathbb{R}^{3} / G \rightarrow \mathbb{R}^{m_{i}}$ be $\lambda$-modular embeddings associated to $\left(\mathcal{C}_{i}, \mathcal{A}_{i}, \mathcal{W}_{i}\right)$, respectively. Then path metrics $\hat{d}_{\theta_{1}}$ and $\hat{d}_{\theta_{2}}$ on $\mathbb{R}^{3} / G$ are bilipschitz equivalent.

Proof. Since $\left(\mathfrak{C}_{1}, \mathcal{A}_{1}, \mathcal{W}_{1}\right)$ and $\left(\mathfrak{C}_{2}, \mathcal{A}_{2}, \mathcal{W}_{2}\right)$ have compatible atlases, there exists $L \geq 1$ so that, for every $H \in \mathcal{C}(\mathcal{X})$,

$$
\tau_{H}=\varphi_{H}^{2} \circ\left(\varphi_{H}^{1}\right)^{-1}:\left(c_{H}^{1}\right)^{\text {diff }} \rightarrow\left(c_{H}^{2}\right)^{\text {diff }}
$$

is $L$-bilipschitz, where $\varphi_{H}^{i}: H^{\text {diff }} \rightarrow\left(c_{H}^{i}\right)^{\text {diff }}$ is the chart for $H$ in $\mathcal{A}_{i}$ for $i=1,2$.

Since $\theta_{1}$ and $\theta_{2}$ are $\lambda$-modular embeddings, we also have constants $L_{1}$ and $L_{2}$ so that $\theta_{i} \circ \pi_{G} \circ\left(\varphi_{H}^{i}\right)^{-1}$ is a $\left(\lambda^{k}, L_{i}\right)$-quasisimilarity for each $H \in \mathcal{C}\left(X_{k}\right)$ and every $k \geq 0$.

Let $H \in \mathcal{C}(\mathcal{X})$. We define

$$
\theta_{H}=\theta_{2} \circ \theta_{1}^{-1} \mid \theta_{1}\left(\pi_{G}\left(H^{\text {diff }}\right)\right): \theta_{1}\left(\pi_{G}\left(H^{\text {diff }}\right)\right) \rightarrow \theta_{2}\left(\pi_{G}\left(H^{\text {diff }}\right)\right) .
$$

Since

$$
\theta_{H}=\left(\theta_{2} \circ \pi_{G} \circ\left(\varphi_{H}^{2}\right)^{-1}\right) \circ \tau_{H} \circ\left(\theta_{1} \circ \pi_{G} \circ\left(\varphi_{H}^{1}\right)^{-1}\right)^{-1},
$$

$\theta_{H}$ is $L L_{1} L_{2}$-bilipschitz.

Let $\Omega=\left(\mathbb{R}^{3} / G\right) \backslash \pi_{G}\left(X_{\infty}\right)$. Since $\theta_{H}$ is uniformly bilipschitz on each $\theta_{1}\left(\pi_{G}\left(H^{\text {diff }}\right)\right)$, we observe that $\theta_{2} \circ \theta_{1}^{-1} \mid \theta_{1}(\Omega)$ is a bilipschitz map in the path metric from $\theta_{1}(\Omega)$ to $\theta_{2}(\Omega)$. Since $\theta_{1}\left(\mathbb{R}^{3} / G\right)$ is the closure of $\theta_{1}(\Omega)$, we observe that $\theta_{2} \circ \theta_{1}^{-1}$ is bilipschitz in the path metric from $\theta_{1}\left(\mathbb{R}^{3} / G\right)$ to $\theta_{2}\left(\mathbb{R}^{3} / G\right)$. The claim now follows. 
We state the modular embedding theorem with respect to a given rigid welding structure as follows.

Theorem 6.2 (Modular embedding theorem). Let $\left(\mathbb{R}^{3} / G, \mathcal{X}\right)$ be a decomposition space of finite type and let $(\mathcal{C}, \mathcal{A}, \mathcal{W})$ be a rigid welding structure on $\mathcal{X}$. Then for every $0<\lambda<1$, there exists a $\lambda$-modular embedding $\theta: \mathbb{R}^{3} / G \rightarrow \mathbb{R}^{n}$ where $n \geq 16$, whose image $\theta\left(\mathbb{R}^{3} / G\right)$ is quasiconvex in the Euclidean metric. Moreover, there exists $L=L(\theta) \geq 1$ so that, any two distinct points $x, y \in \theta\left(\mathbb{R}^{3} / G\right)$ are contained in an L-bilipschitz image of a closed Euclidean 3-ball of radius $|x-y|$.

The proof of the modular embedding theorem is divided into two parts. First we consider a tree Tree $_{\mathcal{X}}$ derived from the combinatorial structure of the defining sequence $\mathcal{X}$ and a bilipschitz embedding of Tree $\mathcal{X}$ into some Euclidean space $\mathbb{R}^{d}$. In the second part, we obtain an embedding of $\mathbb{R}^{3} / G$ into $\mathbb{R}^{16+d}$ by gluing reshaped and rescaled condensers in a rigid welding structure provided by Theorem 5.2. This gluing is guided by the embedded structural tree Tree $\mathcal{X}$.

\subsection{Combinatorial trees}

Let $\mathbb{R}^{3} / G$ be a decomposition space with a defining sequence $\mathcal{X}=\left(X_{k}\right)$. We denote by $\operatorname{Tree}_{\mathcal{X}}$ the tree with vertices $\mathcal{C}(\mathcal{X})$ and unoriented edges $\left\langle H, H^{\prime}\right\rangle$, where $H \in$ $\mathcal{C}\left(X_{k}\right)$ and $H^{\prime} \in \mathcal{C}\left(H \cap X_{k+1}\right)$.

Given $H, H^{\prime} \in \mathcal{C}(\mathcal{X})$, we define

$$
\rho_{\mathcal{X}}\left(H, H^{\prime}\right)=\max \left\{\operatorname{level}\left(H^{\prime \prime}\right) \in \mathbb{Z}: H \cup H^{\prime} \subset H^{\prime \prime} \in \mathcal{C}(\mathcal{X})\right\} .
$$

Since Tree $_{\mathcal{X}}$ is a tree there exists a unique shortest chain $H=H_{1}, \ldots, H_{\ell}=H^{\prime}$ so that $\left\langle H_{i}, H_{i+1}\right\rangle$ is an edge in Tree $\mathcal{X}$ for every $i=1, \ldots, \ell-1$. In particular, there exists a unique index $i_{0}=i_{0}\left(H, H^{\prime}\right)$ so that level $\left(H_{i_{0}}\right)=\rho_{\mathcal{X}}\left(H, H^{\prime}\right)$.

Given $\lambda>0$ we define the metric $\delta_{\lambda}$ on $\operatorname{Tree}_{\mathcal{X}}$ by the formula

$$
\delta_{\lambda}\left(H, H^{\prime}\right)=\sum_{i=1}^{\ell-1} \lambda^{\min \left\{\operatorname{level}\left(H_{i}\right), \operatorname{level}\left(H_{i+1}\right)\right\}},
$$

where $H, H^{\prime} \in \mathcal{C}(\mathcal{X})$ and the sum is taken over the shortest chain $H=H_{1}, \ldots$, $H_{\ell}=H^{\prime}$. The metric $\delta_{1}$ is the standard graph distance on Tree $\mathcal{X}$. The definition of the metric $\delta_{\lambda}$ immediately yields a distance estimate

$$
\lambda^{\rho_{\mathcal{X}}\left(H, H^{\prime}\right)} \leq \delta_{\lambda}\left(H, H^{\prime}\right) \leq C \lambda^{\rho_{\mathcal{X}}\left(H, H^{\prime}\right)}
$$

for all $H, H^{\prime} \in \mathcal{C}(\mathcal{X}), H \neq H^{\prime}$, where $C=C(\lambda)$.

This distance estimate implies that the metric trees $\left(\right.$ Tree $\left._{\mathcal{X}}, \delta_{\lambda}\right), \lambda>0$, are quasisymmetrically equivalent. We record this observation in the following lemma.

Lemma 6.3. Let $\lambda_{1}, \lambda_{2}>0$. The identity map $\left(\operatorname{Tree}_{\mathcal{X}}, \delta_{\lambda_{1}}\right) \rightarrow\left(\operatorname{Tree}_{\mathcal{X}}, \delta_{\lambda_{2}}\right)$ is $\eta$-quasisymmetric with $\eta(t)=C t^{p}$, where $p=\log \lambda_{2} / \log \lambda_{1}$ and $C=C\left(\lambda_{1}, \lambda_{2}\right)$. 
The metric trees $\left(\operatorname{Tree}_{\mathcal{X}}, \delta_{\lambda}\right.$ ) embed bilipschitzly into Euclidean spaces. Recall that $\left(e_{1}, \ldots, e_{n}\right)$ is the standard basis of $\mathbb{R}^{n}$ for $n \geq 1$.

Lemma 6.4. Let $\left(\mathbb{R}^{3} / G, \mathcal{X},(\mathcal{C}, \mathcal{A}, \mathcal{W})\right)$ be a decomposition space of finite type and let $0<\lambda<1$. Then there exist $n=n(\mathcal{X}, \lambda)$ and a map $e_{\mathcal{X}}: \mathcal{C}(\mathcal{X}) \rightarrow\left\{e_{1}, \ldots, e_{n}\right\}$ so that the map $\vartheta:\left(\right.$ Tree $\left._{\mathcal{X}}, \delta_{\lambda}\right) \rightarrow \mathbb{R}^{n}$ defined inductively by $\vartheta\left(X_{0}\right)=0$ and $\vartheta\left(H^{\prime}\right)=$ $\vartheta(H)+\lambda^{k} e_{\mathcal{X}}\left(H^{\prime}\right)$ for $H \in \mathcal{C}\left(X_{k}\right)$ and $H^{\prime} \in \mathcal{C}\left(H \cap X_{k+1}\right)$, is a bilipschitz embedding.

Proof. Let $m_{0}>0$ be the smallest integer satisfying

$$
\sum_{j=1}^{\infty} \lambda^{j m_{0}}<1 / 4
$$

Since $\mathcal{X}$ has finite type, there exists $n$ depending on $\mathcal{C}$ and $m_{0}$, thus depending on $\mathcal{X}$ and $\lambda$, so that

$$
\#\left(\bigcup_{i=k}^{k+2 m_{0}} \mathcal{C}\left(X_{i} \cap H\right)\right) \leq n
$$

for all $k \geq 0$ and $H \in \mathcal{C}\left(X_{k}\right)$. We fix a map $e_{\mathcal{X}}: \mathcal{C}(\mathcal{X}) \rightarrow\left\{e_{1}, \ldots, e_{n}\right\}$ so that if $e_{\mathcal{X}}(H)=e_{\mathcal{X}}\left(H^{\prime}\right)$ then the graph distance $\delta_{1}\left(H, H^{\prime}\right)$ satisfies $\delta_{1}\left(H, H^{\prime}\right) \geq m_{0}$

We show now that the mapping $\vartheta:$ Tree $\mathcal{X} \rightarrow \mathbb{R}^{n}$, defined in the statement, is a bilipschitz embedding.

Let $H, H^{\prime} \in \mathcal{C}(\mathcal{X})$ and let $H=H_{1}, \ldots, H_{\ell}=H^{\prime}$ be the unique shortest chain. Let $I_{j}=\left\{i: 1 \leq i \leq \ell, i \neq i_{0}\left(H, H^{\prime}\right)\right.$ and $\left.e_{\mathcal{X}}\left(H_{i}\right)=e_{j}\right\}$ for $j=1, \ldots, n$. Then

$$
\vartheta(H)-\vartheta\left(H^{\prime}\right)=\sum_{i=1}^{\ell-1} \vartheta\left(H_{i}\right)-\vartheta\left(H_{i+1}\right)=\sum_{j=1}^{n}\left(\sum_{i \in I_{j}} \pm\left(\vartheta\left(H_{i}\right)-\vartheta\left(H_{\tilde{i}}\right)\right)\right),
$$

where $\tilde{i}$ is either $i+1$ or $i-1$ such that level $\left(H_{i}\right)=\operatorname{level}\left(H_{\tilde{i}}\right)+1$. The positive sign is chosen when $\tilde{i}=i+1$, and the negative sign is chosen when $\tilde{i}=i-1$.

By orthogonality,

$$
\left|\vartheta(H)-\vartheta\left(H^{\prime}\right)\right|=\left(\sum_{j=1}^{n}\left|\sum_{i \in I_{j}} \pm\left(\vartheta\left(H_{i}\right)-\vartheta\left(H_{\tilde{i}}\right)\right)\right|^{2}\right)^{1 / 2} .
$$

Since $\vartheta\left(H_{i}\right)-\vartheta\left(H_{\tilde{i}}\right)=\lambda^{\operatorname{level}\left(H_{i}\right)} e_{j}$ for $i \in I_{j}$, we have

$$
\frac{3}{4} \lambda^{k_{j}} \leq\left|\sum_{i \in I_{j}} \pm\left(\vartheta\left(H_{i}\right)-\vartheta\left(H_{\tilde{i}}\right)\right)\right| \leq \frac{5}{2} \lambda^{k_{j}},
$$

where $k_{j}=\min \left\{\operatorname{level}\left(H_{i}\right): i \in I_{j}\right\}$. Since

$$
\rho_{\mathcal{X}}\left(H, H^{\prime}\right)=\min \left\{k_{j}: 1 \leq j \leq n\right\}-1,
$$

we have

$$
\frac{3}{4} \lambda^{\rho \mathcal{X}\left(H, H^{\prime}\right)+1} \leq\left|\vartheta(H)-\vartheta\left(H^{\prime}\right)\right| \leq \frac{5 \sqrt{n}}{2} \lambda^{\rho \mathcal{X}\left(H, H^{\prime}\right)+1} .
$$

Thus, by (6.5), $\vartheta$ is bilipschitz. 


\subsection{Bending and reshaping of condensers}

Suppose that $\mathrm{c}=(A, B)$ is a condenser in a rigid welding structure in $\mathbb{R}^{m}$ for some $m \geq 4$ and that $e: \mathcal{C}(B) \rightarrow\left\{e_{m+1}, \ldots, e_{m+n}\right\}$ is an injection, where $\left(e_{1}, \ldots, e_{m+n}\right)$ is an orthonormal basis of $\mathbb{R}^{m+n}$. We say that a bilipschitz PL-homeomorphism $b_{\mathrm{c}, e}: \mathbb{R}^{m+n} \rightarrow \mathbb{R}^{m+n}$ is a bending of c by $e$ if

1. $b_{\mathrm{c}, e} \mid \partial A=\mathrm{id}$

2. $b_{\mathrm{c}, e} \mid D: x \mapsto x+e(D)$ for every $D \in \mathcal{C}(B)$, and

3. $b_{\mathrm{c}, e}\left(\right.$ int c $\left.\mathrm{c}^{\text {diff }}\right) \subset \mathbb{B}^{3} \times(0,1) \times \mathbb{R}^{m+n-4}$.

Bendings of c by $e$ can be found easily.

Let $k \geq 4$ and $\lambda \in(0,1)$. We define the $\lambda$-reshaping $s_{\lambda}: \mathbb{R}^{k} \rightarrow \mathbb{R}^{k}$ to be

$$
s_{\lambda}(x, t, y)=(c(t) x, t, y)
$$

for $(x, t, y) \in \mathbb{R}^{3} \times \mathbb{R} \times \mathbb{R}^{k-4}$, where

$$
c(t)= \begin{cases}\lambda, & t \geq 1 \\ 1-(1-\lambda) t, & 0 \leq t \leq 1 \\ 1, & t \leq 0\end{cases}
$$

\subsection{Proof of the modular embedding theorem}

To prove Theorem 6.2, we construct first an auxiliary sequence of PL submanifolds $\left(M_{j}\right)$ of a fixed Euclidean space which tends to a PL submanifold $M_{\infty}$. The image of the embedding $\theta \mid \pi_{G}\left(\mathbb{R}^{3} \backslash X_{\infty}\right)$ will be the manifold $M_{\infty}$. This embedding is then extended to $\mathbb{R}^{3} / G$ by continuity.

Assume, as we may by Theorem 5.2 , that $(\mathcal{C}, \mathcal{A}, \mathcal{W})$ is a rigid welding structure for $\mathcal{X}$ in $\mathbb{R}^{16}$.

Auxiliary sequence $\left(M_{j}\right)$. Let $e_{\mathcal{X}}: \mathcal{C}(\mathcal{X}) \rightarrow\left\{e_{16+1}, \ldots, e_{16+n}\right\}$ be the map and let $\vartheta:$ Tree $\mathcal{X} \rightarrow\{0\} \times \mathbb{R}^{n}$ be the embedding defined in Lemma 6.4, with a natural shift of coordinates; recall that $\vartheta\left(X_{0}\right)=0$.

We enumerate the cubes-with-handles in $\mathcal{C}(\mathcal{X})$ by $H_{0}, H_{1}, \ldots$ so that $H_{0}=X_{0}$ and if $H_{i} \in \mathcal{C}\left(X_{k}\right)$ then $H_{i+1} \in \mathcal{C}\left(X_{k}\right) \cup \mathcal{C}\left(X_{k+1}\right)$. We may assume that the condenser $\mathrm{c}_{0}=\left(A_{0}, B_{0}\right)$ is in $\mathcal{C}$ and that the charts $\varphi_{X_{0}}: X_{0}^{\text {diff }} \rightarrow \mathrm{c}_{0}^{\text {diff }}$ are chosen so that $A_{0}=X_{0}$ and $\varphi_{X_{0}} \mid \partial X_{0}=\mathrm{id}$. We write $c_{i}=\left(A_{i}, B_{i}\right)$ for the condensers $\mathrm{c}_{H_{i}} \in \mathcal{C}$ and denote by $\varphi_{i}$ the charts $\varphi_{H_{i}}: H_{i}^{\text {diff }} \rightarrow \mathrm{c}_{i}^{\text {diff }}$ in $\mathcal{A}$ for $i \geq 0$.

Submanifolds $\left(M_{j}\right)$ will be constructed by gluing together bended and reshaped condensers $\left\{\mathrm{c}_{i}: i \geq 0\right\}$ guided by the embedded tree $\vartheta($ Tree $\mathcal{X})$.

We start by defining the directions for bending. Given $i \geq 0$, we denote by $\Phi_{i}: \mathcal{C}\left(H_{i} \cap X_{\text {level }\left(H_{i}\right)+1}\right) \rightarrow \mathcal{C}\left(B_{i}\right)$ the bijection between components induced by charts $\varphi_{H_{i}}$ so that $\Phi_{i}\left|\partial H^{\prime}=\varphi_{H_{i}}\right| \partial H^{\prime}$ for $H^{\prime} \in \mathcal{C}\left(H_{i} \cap X_{\text {level }\left(H_{i}\right)+1}\right)$. Furthermore, 
we define $e^{i}: \mathcal{C}\left(B_{i}\right) \rightarrow\left\{e_{16+1}, \ldots, e_{16+n}\right\}$ by $e^{i}=e_{\mathcal{X}} \circ \Phi_{i}^{-1}$.

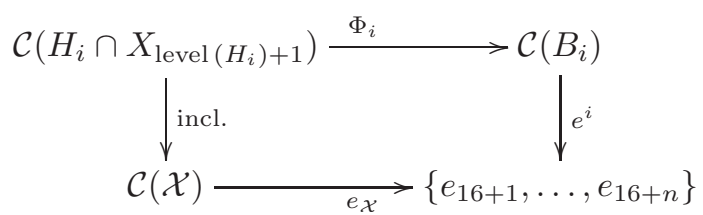

We then fix a family of bendings $\left\{b_{i}=b_{\mathrm{c}_{i}, e^{i}}: \mathbb{R}^{16+m} \rightarrow \mathbb{R}^{16+m}: i \geq 0\right\}$. To ensure that the collection $\left\{b_{i}: i \geq 0\right\}$ of bendings is finite, we require $b_{i}=b_{j}$ when $\left(\mathrm{c}_{i}, e^{i}\right)=\left(\mathrm{c}_{j}, e^{j}\right)$. Thus, the bendings in $\left\{b_{i}, i \geq 0\right\}$ are uniformly bilipschitz.

Fix a constant $C_{0}>0$ so that $B^{16+n}\left(C_{0}\right)$ contains all the condensers in $\mathcal{C}$.

Let $M_{-1}=\left(\mathbb{R}^{3} \backslash X_{0}\right) \times\{0\} \subset \mathbb{R}^{4} \subset \mathbb{R}^{16} \subset \mathbb{R}^{16+n}$ and let $\theta_{-1}: \mathbb{R}^{3} \backslash X_{0} \rightarrow \mathbb{R}^{16+n}$ be the natural inclusion. We define $M_{0}$ by

$$
M_{0}=M_{-1} \cup \mathrm{c}_{0}^{\text {diff }}
$$

and an embedding $\theta_{0}: \mathbb{R}^{3} \backslash X_{1} \rightarrow \mathbb{R}^{16+n}$ by $\theta_{0} \mid \mathbb{R}^{3} \backslash X_{0}=\theta_{-1}$ and $\theta_{0} \mid X_{0}^{\text {diff }}=\varphi_{X_{0}}$.

Suppose now that we have defined manifolds $M_{-1}, \ldots, M_{j-1}$ and embeddings $\theta_{-1}, \ldots, \theta_{j-1}$ so that, for $i=-1, \ldots, j-1$,

1. $M_{i}=M_{i-1} \cup f_{i}\left(\mathrm{c}_{i}^{\text {diff }}\right)$ where $H_{i} \in \mathcal{C}\left(X_{k_{i}}\right), f_{i}$ is the quasisimilarity

$$
x \mapsto \lambda^{k_{i}}\left(s_{\lambda} \circ b_{i}\right)(x)+\vartheta\left(H_{i}\right)+w_{i},
$$

and $w_{i}$ is a point in $\mathbb{R}^{16}$ satisfying $\left|w_{i}\right|<C_{0} \sum_{0 \leq r \leq k_{i}} \lambda^{r}$; and

2. the embedding $\theta_{i}:\left(\mathbb{R}^{3} \backslash X_{0}\right) \cup\left(H_{0}^{\text {diff }} \cup \cdots \cup H_{i}^{\text {diff }}\right) \rightarrow M_{i}$ is defined by $\theta_{i} \mid\left(\mathbb{R}^{3} \backslash\right.$ $\left.X_{0}\right) \cup\left(H_{0}^{\text {diff }} \cup \cdots \cup H_{i-1}^{\text {diff }}\right)=\theta_{i-1}$ and $\theta_{i} \mid H_{i}^{\text {diff }}=f_{i} \circ \varphi_{i}$.

We construct the set $M_{j}$ and the embedding $\theta_{j}$ as follows. Suppose that $H_{j} \in$ $\mathcal{C}\left(X_{k}\right)$ and that $H_{i}, i<j$, is the unique cube-with-handles in $\mathcal{C}\left(X_{k-1}\right)$ with the property $H_{j} \in \mathcal{C}\left(H_{i} \cap X_{k}\right)$, and let $\psi=\varphi_{i} \circ \varphi_{j}^{-1}$ be the welding map from $\mathrm{c}_{j}$ to $\mathrm{c}_{i}^{\text {diff }}$. Since $(\mathcal{C}, \mathcal{A}, \mathcal{W})$ is a rigid structure, $\psi$ is a translation $x \mapsto x+v_{\psi}$ in $\mathbb{R}^{16}$, where $v_{\psi} \in \mathbb{R}^{16},\left\langle v_{\psi}, e_{4}\right\rangle=1$, and $\left|v_{\psi}\right|<C_{0}$ as in the construction. By the induction hypothesis, $f_{i} \mid \varphi_{i}\left(\partial H_{j}\right)$ is a similarity

$$
x \mapsto \lambda^{k} x+\lambda^{k} e_{\mathcal{X}}\left(H_{j}\right)+\vartheta\left(H_{i}\right)+w_{i}=\lambda^{k} x+\vartheta\left(H_{j}\right)+w_{i} ;
$$

here we use the facts that $b_{i} \mid \varphi_{i}\left(\partial H_{j}\right)=e^{i}\left(H_{j}\right)=e_{\mathcal{X}}\left(H_{j}\right)$ and the reshaping $s_{\lambda}$ on $\partial \varphi_{i}\left(H_{j}\right)$ is a scaling by $\lambda$. We set $f_{j}$ to be the quasisimilarity

$$
x \mapsto \lambda^{k}\left(s_{\lambda} \circ b_{j}\right)(x)+\vartheta\left(H_{j}\right)+\lambda^{k} v_{\psi}+w_{i} .
$$

Set also $w_{j}=\lambda^{k} v_{\psi}+w_{i} \in \mathbb{R}^{16}$, and note that by the induction hypothesis that $\left|w_{j}\right| \leq C_{0} \sum_{0 \leq r \leq k} \lambda^{r}$.

Since $\mathrm{c}_{j}$ is a condenser in a rigid structure and $\left\langle v_{\psi}, e_{4}\right\rangle=1$, we have that $M_{j-1} \cap f_{j}\left(\mathrm{c}_{j}\right)$ is a common boundary component of $M_{j-1}$ and $f_{j}\left(\mathrm{c}_{j}\right)$. Thus $M_{j}=$ $M_{j-1} \cup f_{j}\left(\mathrm{c}_{j}\right)$ is a connected manifold with boundary satisfying (1) in the induction hypothesis. 
Now we define the embedding $\theta_{j}:\left(\mathbb{R}^{3} \backslash X_{0}\right) \cup\left(H_{0}^{\text {diff }} \cup \cdots \cup H_{j}^{\text {diff }}\right) \rightarrow M_{j}$ by formula $\theta_{j} \mid \mathbb{R}^{3} \cup\left(H_{0}^{\text {diff }} \cup \cdots \cup H_{j-1}^{\text {diff }}\right)=\theta_{j-1}$ and $\theta_{j} \mid H_{j}^{\text {diff }}=f_{j} \circ \varphi_{j}$. This completes the induction step.

Construction of $M_{\infty}$. Define now the limit manifold $M_{\infty}$ by

$$
M_{\infty}=\bigcup_{j \geq 0} M_{j}
$$

and the limiting embedding $\theta_{\infty}: \mathbb{R}^{3} \backslash X_{\infty} \rightarrow M_{\infty}$ by $\theta_{\infty} \mid M_{j}=\theta_{j}$.

Since there exists $C>0$ so that $\operatorname{diam} \theta(H) \leq C \lambda^{k}$ for every $H \in \mathcal{C}\left(X_{k}\right)$, the components of $\overline{M_{\infty}} \backslash M_{\infty}$ are singletons. Thus $\theta_{\infty} \circ \pi_{G}^{-1}$ extends to a homeomorphism $\theta: \mathbb{R}^{3} / G \rightarrow \overline{M_{\infty}}$.

Note that $\theta \circ \pi_{G} \circ \varphi_{j}^{-1}: \mathrm{c}_{j}^{\text {diff }} \rightarrow \mathbb{R}^{16+n}$ is a $\left(\lambda^{\text {level }\left(H_{j}\right)}, L\right)$-quasisimilarity, for a constant $L \geq 1$ depending only on the family of bendings $\left\{b_{i}: i \geq 0\right\}$ and $n$. Thus $\theta$ is a $\lambda$-modular embedding.

Metric properties of $\theta\left(\mathbb{R}^{3} / G\right)$. We show now the last claim in the statement: given $x, y \in \theta\left(\mathbb{R}^{3} / G\right)$, there exists an $L^{\prime}$-bilipschitz map $h: B^{3}(|x-y|) \rightarrow \theta\left(\mathbb{R}^{3} / G\right)$ so that $x, y \in h\left(B^{3}(|x-y|)\right)$, where $L^{\prime}=L^{\prime}(\theta) \geq 1$. In particular, $\theta\left(\mathbb{R}^{3} / G\right)$ is quasiconvex.

It suffices to consider the case $x, y \in \theta\left(\pi_{G}\left(\mathbb{R}^{3} \backslash X_{\infty}\right)\right)$; the other cases are obtained by similar arguments.

We observe, by the $\left(\lambda^{\text {level }(H)}, L\right)$-quasisimilarity of the mappings $\theta \circ \pi_{G} \circ \varphi_{j}^{-1}$ and the finiteness of condensers in $\mathcal{C}$, the following. If $H$ and $H^{\prime}$ are condensers in $\mathcal{C}(\mathcal{X})$ satisfying $H^{\text {diff }} \cap H^{\prime \text { diff }} \neq \emptyset$, then any two points $x$ and $y$ in $\theta\left(\pi_{G}\left(H^{\text {diff }} \cup H^{\prime \text { diff }}\right)\right)$ can be connected by a PL-curve contained in a 3 -cell in $\theta\left(\pi_{G}\left(H^{\text {diff }} \cup H^{\prime \text { diff }}\right)\right)$ that is $L^{\prime}$-bilipschitz equivalent to a Euclidean ball of diameter $|x-y|$, where $L^{\prime}$ depends only on the data. In particular, the claim holds in this case.

We now assume $x \in \theta\left(\pi_{G}\left(H^{\text {diff }}\right)\right), y \in \theta\left(\pi_{G}\left(H^{\text {diff }}\right)\right)$ and $H^{\text {diff }} \cap H^{\prime \text { diff }}=\emptyset$. Let $H=H_{0}, \ldots, H_{\ell}=H^{\prime}$ be the unique shortest chain in Tree $\mathcal{X}$ joining vertices $H$ and $H^{\prime}$, and $\rho_{\mathcal{X}}\left(H, H^{\prime}\right)=\min \left\{\operatorname{level}(\hat{H}) \in \mathbb{Z}: H \cup H^{\prime} \subset \hat{H} \in \mathcal{C}(\mathcal{X})\right\}$. Then by the construction of the embedding $\theta$, there exists $C=C(\theta) \geq 1$ so that

$$
C^{-1} \lambda^{\rho_{\mathcal{X}}\left(H, H^{\prime}\right)} \leq|x-y| \leq C \lambda^{\rho_{\mathcal{X}}\left(H, H^{\prime}\right)} .
$$

There exist $C^{\prime}=C^{\prime}(\theta) \geq 1$ and points $x=x_{0}, \ldots, x_{\ell}=y$ with $x_{i} \in \theta\left(\pi_{G}\left(H_{i}^{\text {diff }}\right)\right)$ so that each $x_{i}, 1 \leq i \leq \ell-1$, is contained in a 3-cell $D_{i} \subset \theta\left(\pi_{G}\left(H_{i}^{\text {diff }}\right)\right)$ which is $L^{\prime}$-bilipschitz equivalent to $B^{3}\left(\lambda^{\operatorname{level}\left(H_{i}\right)}\right)$, and so that

$$
C^{\prime-1} \lambda^{\operatorname{level}\left(H_{i}\right)} \leq\left|x_{i}-x_{i+1}\right| \leq C \lambda^{\operatorname{level}\left(H_{i}\right)}
$$

for $0 \leq i \leq \ell-1$. Consequently,

$$
\sum_{i=0}^{\ell-1}\left|x_{i}-x_{i+1}\right| \leq C|x-y| .
$$

By the argument for the previous case, we find PL 3-cells $E_{i} \subset \pi_{G}\left(H_{i}^{\text {diff }} \cup H_{i+1}^{\text {diff }}\right)$ that are $L^{\prime}$-bilipschitz equivalent to $B^{3}\left(\left|x_{i}-x_{i+1}\right|\right)$ and contain the points $x_{i}$ and 
$x_{i+1}$ in their interiors in $\pi_{G}\left(H_{i}^{\text {diff }} \cup H_{i+1}^{\text {diff }}\right)$, respectively. It is now easy to find a PL 3-cell $E \subset \bigcup_{i=1}^{\ell-1} D_{i} \cup \bigcup_{i=0}^{\ell-1} E_{i}$ that is $L^{\prime}$-bilipschitz equivalent to $B^{3}(|x-y|)$ and contains the points $x$ and $y$. This concludes the proof of Theorem 6.2.

Remark 6.5. The fact that any two points $x, y$ in $\theta\left(\mathbb{R}^{3} / G\right)$ are contained in a 3-cell in $\theta\left(\mathbb{R}^{3} / G\right)$ that is $L$-bilipschitz equivalent to a Euclidean ball of diameter $|x-y|$, yields that $\theta\left(\mathbb{R}^{3} / G\right)$ has the Loewner property. We formulate this more precisely in Section 7.4 .

\section{Semmes spaces}

In this section we discuss quasiconvexity, Ahlfors regularity, linearly locally contractibility, and the Loewner property of the modular metrics provided by the modular embedding theorem as listed in Theorem 1.2.

Definition 7.1. Let $\left(\mathbb{R}^{3} / G, \mathcal{X}\right)$ be a decomposition space of finite type, let $(\mathcal{C}, \mathcal{A}, \mathcal{W})$ be a rigid welding structure for $\mathcal{X}$, let $\theta: \mathbb{R}^{3} / G \rightarrow \mathbb{R}^{n}$ be a modular embedding associated to $(\mathcal{C}, \mathcal{A}, \mathcal{W})$ as in Theorem 6.2 , and let $d_{\theta}$ be a $\lambda$-modular metric associated to $\theta$. A metric space $\left(\mathbb{R}^{3} / G, d_{\lambda}\right)$ is called a Semmes space if $d_{\lambda}$ is bilipschitz equivalent to $d_{\theta}$. In this case we say $d_{\lambda}$ is a Semmes metric with a scaling factor $\lambda$.

At times we say that $\left(\mathbb{R}^{3} / G, \mathcal{X},(\mathcal{C}, \mathcal{A}, \mathcal{W}), \theta, d_{\lambda}\right)$ is a Semmes space in order to emphasize the relation to between the structure, the embedding, and the metric.

Product spaces $\mathbb{R}^{3} / G \times \mathbb{R}^{m}$ carry the natural product metric $d_{\lambda, m}$ defined by

$$
d_{\lambda, m}((x, u),(y, v))=d_{\lambda}(x, y)+|u-v|
$$

for $(x, u)$ and $(y, v)$ in $\mathbb{R}^{3} / G \times \mathbb{R}^{m}$.

We observe that the metric space $\left(\mathbb{R}^{3} / G, d_{\lambda}\right)$ is quasiconvex; indeed, $\theta\left(\mathbb{R}^{3} / G\right)$ is a quasiconvex set in $\mathbb{R}^{n}$ by Theorem 6.2. By quasiconvexity and Lemma 6.1 we have the bilipschitz equivalence of the modular metric spaces associated to rigid welding structures with compatible atlases. We record this observation as a lemma.

Lemma 7.2. Let $\lambda \in(0,1)$ and suppose that $\left(\mathbb{R}^{3} / G, \mathcal{X},\left(\mathcal{C}_{i}, \mathcal{A}_{i}, \mathcal{W}_{i}\right), \theta_{i}, d_{\theta_{i}}\right)$ are $\lambda$-modular metric spaces, $i=1,2$. The metrics $d_{\theta_{1}}$ and $d_{\theta_{2}}$ are bilipschitz equivalent if the rigid welding structures $\left(\mathcal{C}_{1}, \mathcal{A}_{1}, \mathcal{W}_{1}\right)$ and $\left(\mathcal{C}_{2}, \mathcal{A}_{2}, \mathcal{W}_{2}\right)$ have compatible atlases.

\subsection{Metric properties}

We list some elementary metric and measure theoretic properties of Semmes spaces in the following remarks and the subsequent lemma. Let $\left(\mathbb{R}^{3} / G, \mathcal{X},(\mathcal{C}, \mathcal{A}, \mathcal{W}), \theta, d_{\lambda}\right)$ be a Semmes space.

Remark 7.3. By quasiconvexity of $d_{\theta}$, the path metric space $\left(\mathbb{R}^{3} / G, \hat{d}_{\theta}\right)$ is a Semmes space. Similarly, the path metric space $\left(\mathbb{R}^{3} / G, \hat{d}_{\lambda}\right)$ of $\left(\mathbb{R}^{3} / G, d_{\lambda}\right)$ is a Semmes space. 
Remark 7.4. By modularity of the embedding $\theta$ and quasiconvexity of the metric $d_{\theta}$ there exists a constant $C=C\left(d_{\lambda}\right)$ so that

$$
C^{-1} \lambda^{\rho_{\mathcal{X}}\left(H, H^{\prime}\right)} \leq d_{\lambda}(x, y) \leq C \lambda^{\rho_{\mathcal{X}}\left(H, H^{\prime}\right)}
$$

for $x \in \pi_{G}\left(H^{\text {diff }}\right)$ and $y \in \pi_{G}\left(H^{\prime \text { diff }}\right)$ whenever $H, H^{\prime} \in \mathcal{C}(\mathcal{X})$ and $H^{\text {diff }} \cap H^{\prime \text { diff }}=\emptyset$.

Remark 7.5. By the finiteness of the welding structure $(\mathcal{C}, \mathcal{A}, \mathcal{W})$ and the quasisimilarity property (6.1) of modular embeddings, there exists $C>1$ so that for every $k \geq 0$ and $H \in \mathcal{C}\left(X_{k}\right)$,

1. $C^{-1} \lambda^{k} \leq \operatorname{dist}_{d_{\lambda}}\left(\partial \pi_{G}(H), \partial \pi_{G}\left(H^{\prime}\right)\right) \leq C \lambda^{k}$, if $H^{\prime} \in \mathcal{C}(\mathcal{X}), H^{\prime} \subset H$ and $H^{\prime} \neq H$;

2. $C^{-1} \lambda^{k} \leq \operatorname{diam}_{d_{\lambda}} \pi_{G} H^{\text {diff }} \leq C \lambda^{k}$;

3. $C^{-1} \lambda^{3 k} \leq \mathcal{H}_{d_{\lambda}}^{3}\left(\pi_{G}\left(H^{\text {diff }}\right)\right) \leq C \lambda^{3 k}$; and

4. $C^{-1} r^{3} \leq \mathcal{H}_{d_{\lambda}}^{3}\left(B_{d_{\lambda}}(x, r)\right) \leq C r^{3}$, if $B_{d_{\lambda}}(x, r) \subset \pi_{G}\left(X_{k-1} \backslash X_{k+2}\right)$

Observe also that components of $\pi_{G}\left(X_{\infty}\right)$ are singletons in $\left(\mathbb{R}^{3} / G, d_{\lambda}\right)$. Thus $\pi_{G}\left(X_{\infty}\right)$ is 0-dimensional.

Lemma 7.6. Let $\left(\mathbb{R}^{3} / G, \mathcal{X},(\mathcal{C}, \mathcal{A}, \mathcal{W}), \theta, d_{\lambda}\right)$ be a Semmes space. Then there exists $C>1$ so that

$$
C^{-1} \lambda^{k} \leq \operatorname{diam}_{d_{\lambda}} \pi_{G} H \leq C \lambda^{k},
$$

for every $k \geq 0$ and $H \in \mathcal{C}\left(X_{k}\right)$. that

If, in addition, $\lambda^{3} \gamma_{\mathcal{X}}<1$, then $\mathcal{H}_{d_{\lambda}}^{3}\left(\pi_{G}\left(X_{\infty}\right)\right)=0$ and there exists $C>1$ so

$$
C^{-1} \lambda^{3 k} \leq \mathcal{H}_{d_{\lambda}}^{3}\left(\pi_{G} H\right) \leq C \lambda^{3 k}
$$

for every $k \geq 0$ and $H \in \mathcal{C}\left(X_{k}\right)$.

Proof. Since

$$
\pi_{G} H=\overline{\bigcup_{i \geq k} \bigcup_{H^{\prime} \in \mathcal{C}\left(X_{i} \cap H\right)} \pi_{G}\left(H^{\prime \text { diff }}\right)} .
$$

we have, by connectedness and Remark $7.5(2)$,

$$
C^{-1} \lambda^{k} \leq \operatorname{diam}_{d_{\lambda}} H^{\text {diff }} \leq \operatorname{diam}_{d_{\lambda}} H \leq \sum_{i \geq k} C \lambda^{i} \leq C^{\prime} \lambda^{k} .
$$

Similarly, we have that

$$
\mathcal{H}_{d_{\lambda}}^{3}\left(\pi_{G}(H)\right)=\mathcal{H}_{d_{\lambda}}^{3}\left(\pi_{G}\left(X_{\infty} \cap H\right)\right)+\sum_{i \geq k} \sum_{H^{\prime} \in \mathcal{C}\left(X_{i} \cap H\right)} \mathcal{H}_{d_{\lambda}}^{3}\left(\pi_{G}\left(H^{\prime \text { diff }}\right)\right) .
$$

Suppose now that $\lambda^{3} \gamma_{\mathcal{X}}<1$. Then, by (7.2),

$$
\mathcal{H}_{d_{\lambda}}^{3}\left(\pi_{G}\left(X_{\infty}\right)\right) \leq \limsup _{i \rightarrow 0} \sum_{H^{\prime} \in \mathcal{C}\left(X_{i}\right)}\left(C \lambda^{i}\right)^{3} \leq C^{3} \limsup _{i \rightarrow 0} \lambda^{3 i} \gamma_{\mathcal{X}}^{i}=0 .
$$


By Definition 4.2 and Remark $7.5(3)$, there exist $k_{0} \geq 1$ and $C>1$ so that

$$
C^{-1} \lambda^{3 k} \leq \sum_{i \geq k} \sum_{H^{\prime} \in \mathcal{C}\left(X_{i} \cap H\right)} \mathcal{H}_{d_{\lambda}}^{3}\left(\pi_{G}\left(H^{\prime \text { diff }}\right)\right) \leq \sum_{i \geq k} C \lambda^{3 i} \gamma_{\mathcal{X}}^{i-k} \leq C \lambda^{3 k}
$$

for $k \geq k_{0}$. After replacing $C$ by another constant that depends only on $C, \lambda, k_{0}$, and the upper growth $\bar{\gamma}_{\mathcal{X}}$, we may obtain (7.3) for all $k \geq 0$. This concludes the proof.

Remark 7.7. We observe that, by Remark 7.5 and Lemma 7.6, the number

$$
\hat{\epsilon}_{\lambda}=\min _{k \geq 0} \min _{H \in \mathcal{C}\left(X_{k}\right)}\left\{\frac{\operatorname{dist}_{d_{\lambda}}\left(\pi_{G}(\partial H), \pi_{G}\left(H \backslash H^{\text {diff }}\right)\right)}{\lambda^{k}}\right\} .
$$

is strictly positive. Furthermore, we may fix $\varepsilon_{\lambda}=\varepsilon_{\lambda}\left(d_{\lambda}\right)<\hat{\varepsilon} / 10$ so that the neighborhood $N_{d_{\lambda}}\left(\pi_{G}(\partial H), \epsilon_{\lambda} \lambda^{\text {level }(H)}\right)$ is contained in a regular neighborhood of $\pi_{G}(\partial H)$ in $\pi_{G}\left(X_{\text {level }(H)-1}\right) \backslash \pi_{G}\left(H_{\text {level }(H)+1}\right)$.

\subsection{Ahlfors regularity}

The Ahlfors regularity of Semmes spaces follows as in [19], Lemma 3.45. We discuss the details for completeness of the exposition.

Proposition 7.8. Let $\left(\mathbb{R}^{3} / G, \mathcal{X},(\mathcal{C}, \mathcal{A}, \mathcal{W}), \theta, d_{\lambda}\right)$ be a Semmes space, and suppose that $0<\lambda^{3} \gamma_{\mathcal{X}}<1$. Then the space $\left(\mathbb{R}^{3} / G, d_{\lambda}\right)$ is Ahlfors 3 -regular, and the spaces $\left(\mathbb{R}^{3} / G \times \mathbb{R}^{m}, d_{\lambda, m}\right)$ are Ahlfors $(3+m)$-regular for $m \geq 1$.

Proof. It suffices to show that $\left(\mathbb{R}^{3} / G, d_{\lambda}\right)$ is Ahlfors 3-regular. Then the Ahlfors $(3+m)$-regularity of spaces $\left(\mathbb{R}^{3} / G \times \mathbb{R}^{m}, d_{\lambda, m}\right), m \geq 1$, follows by taking products.

By the bilipschitz invariance of Ahlfors regularity, we may assume that $d_{\lambda}$ is the metric $d_{\theta}$ defined by a $\lambda$-modular embedding $\theta: \mathbb{R}^{3} / G \rightarrow \mathbb{R}^{16+n}$. To simplify the exposition, we assume that $X_{0}=\mathbb{B}^{3}$, and set $X_{-j}=B^{3}\left(0, \lambda^{-j}\right)$ for $j>0$.

To show that

$$
C^{-1} r^{3} \leq \mathcal{H}_{d_{\lambda}}^{3}\left(B_{d_{\lambda}}(x, r)\right) \leq C r^{3}
$$

for all balls $B_{d_{\lambda}}(x, r)$ in $\left(\mathbb{R}^{3} / G, d_{\lambda}\right)$, we consider two cases: (a) $x \in \pi_{G}\left(\mathbb{R}^{3} \backslash X_{\infty}\right)$, and (b) $x \in \pi_{G}\left(X_{\infty}\right)$.

We first consider case (a). Assume that $x \in \pi_{G}\left(X_{0} \backslash X_{\infty}\right)$, and suppose $x \in$ $\pi_{G}\left(H^{\text {diff }}\right)$ and $H \in \mathcal{C}\left(X_{k}\right)$. By Remark 7.5(1), there exists a constant $C_{1}=$ $C_{1}\left(d_{\lambda}\right) \in(0,1)$ so that if $r \leq C_{1} \lambda^{k}$ then $B_{d_{\lambda}}(x, r) \subset \pi_{G}\left(X_{k-1} \backslash X_{k+2}\right)$. By (7.2) of Lemma 7.6, there exists a constant $C_{2}=C_{2}\left(d_{\lambda}\right)>1$ so that if $r \geq C_{2} \lambda^{k}$ then $\pi_{G}(H) \subset B_{d_{\lambda}}(x, r)$.

Case (b) follows from (a). Indeed, since $\pi_{G}\left(\mathbb{R}^{3} \backslash X_{\infty}\right)$ is dense in $\mathbb{R}^{3} / G$, given $x \in \pi_{G}\left(X_{\infty}\right)$ and $r>0$ there exists $y \in \pi_{G}\left(\mathbb{R}^{3} \backslash X_{\infty}\right)$ so that $d_{\lambda}(x, y)<r / 2$. So $B_{d_{\lambda}}(y, r / 2) \subset B_{d_{\lambda}}(x, r) \subset B_{d_{\lambda}}(y, 2 r)$, and (7.4) follows by (a).

For $0<r \leq C_{1} \lambda^{k}$, the claim follows from Remark $7.5(4)$. 
For $r \geq C_{2} \lambda^{k}$, we fix $m \in \mathbb{Z}$ so that $\lambda^{m+1} \leq r<\lambda^{m}$. Then, by Remark 7.5 (1), there exist an integer $C_{3}=C_{3}\left(d_{\lambda}\right)>0$, and cubes-with-handles $H^{\prime} \in \mathcal{C}\left(X_{m+C_{3}}\right)$ and $H^{\prime \prime} \in \mathcal{C}\left(X_{m-C_{3}}\right)$ so that

$$
\pi_{G}\left(H^{\prime}\right) \subset B_{d_{\lambda}}(x, r) \subset \pi_{G}\left(H^{\prime \prime}\right) .
$$

Then, by (7.3) in Lemma 7.6, there exists $C=C\left(d_{\lambda}\right)>1$ so that

$$
C^{-1} \lambda^{3\left(m+C_{3}\right)} \leq \mathcal{H}_{d_{\lambda}}^{3}\left(B_{d_{\lambda}}(x, r)\right) \leq C \lambda^{3\left(m-C_{3}\right)} .
$$

In the remaining subcase $C_{1} \lambda^{k}<r<C_{2} \lambda^{k}, B_{d_{\lambda}}(x, r)$ contains the ball $B_{d_{\lambda}}\left(x, C_{1} \lambda^{k}\right)$ and is contained in a cube-with-handles in $\mathcal{C}\left(X_{k-C_{4}}\right)$ for some $C_{4}=$ $C_{4}\left(d_{\lambda}\right)>0$. Then (7.4) follows by combining Remark 7.5 and (7.3) in Lemma 7.6. This concludes the proof.

\subsection{Linear local contractibility}

In this section we show that a Semmes space $\left(\mathbb{R}^{3} / G,\left(X_{k}\right)_{k \geq 0},(\mathcal{C}, \mathcal{A}, \mathcal{W}), \theta, d_{\lambda}\right)$ is linearly locally contractible if $\mathcal{X}$ is locally contractible. Recall that a defining sequence $\mathcal{X}=\left(X_{k}\right)$ is locally contractible if components of $X_{k+1}$ are contractible in $X_{k}$ for $k \geq 0$.

The linear local contractibility of $\left(\mathbb{R}^{3} / G, d_{\lambda}\right)$ is a necessary condition for the existence of a quasisymmetric parametrization of $\left(\mathbb{R}^{3} / G, d_{\lambda}\right)$ by a Euclidean space.

Proposition 7.9. Let $\left(\mathbb{R}^{3} / G, \mathcal{X},(\mathcal{C}, \mathcal{A}, \mathcal{W}), \theta, d_{\lambda}\right)$ be a Semmes space having locally contractible defining sequence $\mathcal{X}$. Then, for every $m \geq 0,\left(\mathbb{R}^{3} / G \times \mathbb{R}^{m}, d_{\lambda, m}\right)$ is linearly locally contractible.

As before, we assume as we may that $X_{0}=\mathbb{B}^{3}$ and $X_{-j}=B^{3}\left(0, \lambda^{-j}\right)$ for $j>0$.

Proof. Since $X_{-k}$ is a 3 -cell for $k \geq 0$, the components of $\pi_{G}\left(X_{k+1}\right)$ are contractible in $\pi_{G}\left(X_{k}\right)$ for every $k \in \mathbb{Z}$ by Lemma 3.1.

Special case: Assume that $m=0$ and $y \in \pi_{G}\left(\mathbb{R}^{3} \backslash X_{\infty}\right)$; so $y \in H^{\text {diff }}$ for some $H \in \mathcal{C}\left(X_{k}\right)$. We consider this case in two parts.

By the uniform quasisimilarity of the modular embedding $\theta$, there exist constants $C_{0}=C_{0}\left(d_{\lambda}\right)>1$ and $C_{1}=C_{1}\left(d_{\lambda}\right)>0$ with the property: if $0<r<C_{1} \lambda^{k}$ there exists a 3-cell $E \subset \pi_{G}\left(X_{k-1} \backslash X_{k+2}\right)$ satisfying

$$
B_{d_{\lambda}}(y, r) \subset E \subset B_{d_{\lambda}}\left(y, C_{0} r\right) .
$$

Hence $B_{d_{\lambda}}(y, r)$ contracts in $B_{d_{\lambda}}\left(y, C_{0} r\right)$ if $r<C_{1} \lambda^{k}$.

Suppose now that $r \geq C_{1} \lambda^{k}$. We fix cubes-with-handles $H^{\prime}, H^{\prime \prime} \in \mathcal{C}(\mathcal{X})$ satisfying $H \subset H^{\prime} \subset H^{\prime \prime}$,

$$
\left.\operatorname{level}\left(H^{\prime}\right)=\min \left\{\operatorname{level}(K): K \in \mathcal{C}(\mathcal{X}), B_{d_{\lambda}}(x, r) \subset \pi_{G}(K)\right)\right\},
$$

and

$$
\operatorname{level}\left(H^{\prime \prime}\right)=\operatorname{level}\left(H^{\prime}\right)-1
$$


Then $B_{d_{\lambda}}(y, r) \subset \pi_{G}\left(H^{\prime}\right)$ and, by Lemma $3.1, \pi_{G}\left(H^{\prime}\right)$ contracts in $\pi_{G}\left(H^{\prime \prime}\right)$. By Remark 7.5 and Lemma 7.6, there exists $C_{2}=C_{2}\left(d_{\lambda}\right) \geq 1$ so that $\operatorname{diam}_{d_{\lambda}}\left(\pi_{G}\left(H^{\prime \prime}\right)\right) \leq$ $C_{2} r$. Thus $\pi_{G}\left(H^{\prime \prime}\right) \subset B_{d_{\lambda}}\left(y, C_{2} r\right)$, and $B_{d_{\lambda}}(y, r)$ contracts in $B_{d_{\lambda}}\left(y, C_{2} r\right)$. This concludes the proof of this special case.

General case: Let $x=(y, v) \in \pi_{G}\left(\mathbb{R}^{3}\right) \times \mathbb{R}^{m}$, where $m \geq 0$. Let $r>0$. To show that there exists $C=C\left(d_{\lambda, m}\right)>1$ so that every ball $B_{d_{\lambda, m}}(x, r)$ is contractible in $B_{d_{\lambda, m}}(x, C r)$, we consider the two cases (a) $x \in \pi_{G}\left(\mathbb{R}^{3} \backslash X_{\infty}\right) \times \mathbb{R}^{m}$ and (b) $x \in \pi_{G}\left(X_{\infty}\right) \times \mathbb{R}^{m}$.

We consider first case (a), that is $x=(y, v) \in \pi_{G}\left(\mathbb{R}^{3} \backslash X_{\infty}\right) \times \mathbb{R}^{m}$ with $y \in \pi_{G}\left(H^{\text {diff }}\right)$ and $H \in C\left(X_{k}\right)$. Then $B_{d_{\lambda, m}}(x, r)$ contracts in $B_{d_{\lambda}}(y, C r) \times$ $\left(v+[-r, r]^{m}\right)$. Thus $B_{d_{\lambda, m}}(x, r)$ is contractible in $B_{d_{\lambda, m}}(x,(C+\sqrt{m}) r)$ and the claim follows.

Case (b) follows from (a). Indeed, there exists $z \in \pi_{G}\left(\mathbb{R}^{3} \backslash X_{\infty}\right) \times \mathbb{R}^{m}$ so that $d_{\lambda, m}(x, z)<r / 2$. Hence $B_{d_{\lambda, m}}(x, r)$ is contained in a ball $B_{d_{\lambda, m}}(z, 2 r)$ that is contractible in $B_{d_{\lambda, m}}(z, 2 C r) \subset B_{d_{\lambda, m}}(x, 4 C r)$, where $C=C\left(d_{\lambda, m}\right)$ is as in case (a).

\subsection{Loewner property}

In this section, we briefly list some other analytical properties of Semmes spaces. We refer to [19], [18], and [9] for definitions and background. Assume in what follows that $\left(\mathbb{R}^{3} / G, d_{\lambda}\right)$ is Ahlfors 3-regular.

From the proof of the modular embedding theorem (Theorem 6.2) we see that any pair of points $x, y \in \mathbb{R}^{3} / G$ is contained in a uniformly bilipschitz image of the Euclidean ball $B^{3}(|x-y|)$. This property is the same as in Lemma 3.70 of [19] for self-similar spaces. The argument of Proposition 10.8 in [19] can now be applied almost verbatim to show that $\left(\mathbb{R}^{3} / G, d_{\lambda}\right)$ supports a $(1,1)$-Poincaré inequality as formulated in (10.9) of [19]. Since the space $\mathbb{R}^{3} / G$ is PL outside $\pi_{G}\left(X_{\infty}\right)$, the Poincaré inequality can be formulated in terms of generalized gradients (upper gradients). We refer to Appendix $\mathrm{C}$ of [18] for a detailed treatment.

Ahlfors 3-regularity, quasiconvexity, and the (1,1)-Poincaré inequality together imply that $\left(\mathbb{R}^{3} / G, d_{\lambda}\right)$ is a Loewner space in the sense of Heinonen and Koskela; see Theorem 5.7 in [9]. A metric measure space $(X, d, \mu)$ of Hausdorff dimension $Q$ is a Loewner space if there exists a function $\phi:(0, \infty) \rightarrow(0, \infty)$ so that

$$
\operatorname{Mod}_{Q}(E, F) \geq \phi(\Delta(E, F, X))
$$

whenever $E$ and $F$ are disjoint continua in $X$, where

$$
\Delta(E, F, X)=\frac{\operatorname{dist}(E, F)}{\min \{\operatorname{diam} E, \operatorname{diam} F\}},
$$

and $\operatorname{Mod}_{Q}(E, F)$ is the $Q$-modulus of the family of paths connecting $E$ and $F$ in $X$.

Suppose now that the space $\left(\mathbb{R}^{3} / G \times \mathbb{R}^{m}, d_{\lambda, m}\right)$ is Ahlfors $(3+m)$-regular and homeomorphic to $\mathbb{R}^{3+m}$ for some $m \geq 0$. Then $\left(\mathbb{R}^{3} / G \times \mathbb{R}^{m}, d_{\lambda, m}\right)$ supports a 
(1, 1)-Poincaré inequality by a theorem of Semmes for manifolds (Theorem B.10 (b) in [18]). Thus $\left(\mathbb{R}^{3} / G \times \mathbb{R}^{m}, d_{\lambda, m}\right)$ is a Loewner space by the aforementioned theorem of Heinonen and Koskela. Recall that the Loewner property is a necessary condition for the quasisymmetric parametrizability; see Tyson [22].

\subsection{Quasisymmetric equivalence of Semmes metrics}

In this section we prove the quasisymmetric equivalence of the Semmes metrics on $\left(\mathbb{R}^{3} / G, \mathcal{X}\right)$ associated to different welding structures and scaling factors.

Proposition 7.10. Let $\left(\mathbb{R}^{3} / G, \mathcal{X},\left(\mathcal{C}_{i}, \mathcal{A}_{i}, \mathcal{W}_{i}\right), \theta_{i}, d_{\lambda_{i}}\right)$ be two Semmes spaces with $i=1,2$ and $\lambda_{1}, \lambda_{2} \in(0,1)$. Suppose that $\left(\mathcal{C}_{1}, \mathcal{A}_{1}, \mathcal{W}_{1}\right)$ and $\left(\mathcal{C}_{2}, \mathcal{A}_{2}, \mathcal{W}_{2}\right)$ have compatible atlases. Then $\mathrm{id}:\left(\mathbb{R}^{3} / G, d_{\lambda_{1}}\right) \rightarrow\left(\mathbb{R}^{3} / G, d_{\lambda_{2}}\right)$ is quasisymmetric.

Proof. Assume, as we may, that $X_{0}=\mathbb{B}^{3}$ and define $X_{-j}=B^{3}\left(0, \lambda^{-j}\right)$ for $j>0$.

Since $\pi_{G}\left(\mathbb{R}^{3} \backslash X_{\infty}\right)$ is dense in $\mathbb{R}^{3} / G$ and the metrics $d_{\lambda_{i}}$ are bilipschitz equivalent to modular metrics $d_{\theta_{i}}$ for $i=1,2$, respectively, it suffices to show that there exists a homeomorphism $\eta:[0, \infty) \rightarrow[0, \infty)$ so that, for all distinct points $x, y$, and $z$ in $\pi_{G}\left(\mathbb{R}^{3} \backslash X_{\infty}\right)$,

$$
\frac{\left|\theta_{2}(x)-\theta_{2}(y)\right|}{\left|\theta_{2}(x)-\theta_{2}(z)\right|} \leq \eta\left(\frac{\left|\theta_{1}(x)-\theta_{1}(y)\right|}{\left|\theta_{1}(x)-\theta_{1}(z)\right|}\right)
$$

We divide the proof into different cases depending on the relative distances between the points $x, y$, and $z$. For brevity, say that points $x$ and $y$ in $\pi_{G}\left(\mathbb{R}^{3} \backslash X_{\infty}\right)$ are close if there exist $H, H^{\prime} \in \mathcal{C}(\mathcal{X})$ so that $\{x, y\} \subset \pi_{G}\left(H^{\text {diff }} \cup H^{\prime \text { diff }}\right)$ and the common boundary $H^{\text {diff }} \cap H^{\prime \text { diff }} \neq \emptyset$. Otherwise, we say that points $x$ and $y$ are far.

Let $x, y$, and $z$ be distinct points in $\pi_{G}\left(\mathbb{R}^{3} \backslash X_{\infty}\right)$.

Case I: Suppose that at least two pairs of points in the set $\{x, y, z\}$ are close.

Then there exist $H, H^{\prime}, H^{\prime \prime} \in \mathcal{C}(\mathcal{X})$ so that $H^{\text {diff }} \cap H^{\prime \text { diff }} \neq \emptyset, H^{\prime \text { diff }} \cap H^{\prime \prime \text { diff }}$ $\neq \emptyset$, and $\{x, y, z\} \subset \pi_{G}\left(H^{\text {diff }} \cup H^{\text {diff }} \cup H^{\prime \prime \text { diff }}\right)$. Then, by quasiconvexity of the metrics $d_{\lambda_{i}}$, compatibility of the atlases, and modularity of the embeddings $\theta_{i}$, there exists $C_{1}=C_{1}\left(\theta_{1}, \theta_{2}\right)>0$ so that (7.5) holds with $\eta=\eta_{1}$, where $\eta_{1}(t)=C_{1} t$.

Case II: Suppose that the points $x, y$, and $z$ are far from each other. Then, by Remark 7.4, there exists $C_{2}=C_{2}\left(\theta_{1}, \theta_{2}\right)>0$ so that (7.5) holds with $\eta=\eta_{2}$, where $\eta_{2}(t)=C_{2} t^{p}$ and $p=\log \lambda_{2} / \log \lambda_{1}$.

Case III: Suppose now that there exists only one pair in $\{x, y, z\}$ where the points are close and that points in the other two pairs are far. We have three subcases.

Case III.1: Suppose that $y$ and $z$ are close. Then $x$ and $y$ are far and $x$ and $z$ are far. So there exists $C=C\left(\theta_{1}, \theta_{2}\right)>0$ so that

$$
\frac{1}{C} \leq \frac{\left|\theta_{i}(x)-\theta_{i}(y)\right|}{\left|\theta_{i}(x)-\theta_{i}(z)\right|} \leq C
$$

for $i=1,2$. Thus (7.5) holds with $\eta=\eta_{3}$, where $\eta_{3}(t)=C_{3} t$ with $C_{3}=$ $C_{3}\left(\theta_{1}, \theta_{2}\right)>0$. 
Case III.2: Suppose now that $x$ and $z$ are close and let $H, H^{\prime} \in \mathcal{C}(\mathcal{X})$ be such that $\{x, z\} \subset \pi_{G}\left(H^{\text {diff }} \cup H^{\text {diff }}\right)$ and $H^{\text {diff }} \cap H^{\prime \text { diff }} \neq \emptyset$. Then, by modularity of the embeddings $\theta_{1}$ and $\theta_{2}$, there exist $C=C\left(\theta_{1}, \theta_{2}\right)>1$ and $w \in \pi_{G}\left(H^{\text {diff }} \cup H^{\prime \text { diff }}\right)$ so that

$$
\min \left\{\left|\theta_{i}(x)-\theta_{i}(w)\right|,\left|\theta_{i}(z)-\theta_{i}(w)\right|\right\} \geq \frac{1}{C} \operatorname{diam} \theta_{i}\left(\pi_{G}\left(H^{\text {diff }} \cup H^{\prime \text { diff }}\right)\right)
$$

for $i=1,2$.

Following the argument for cases I and II, there exists $C_{4}=C_{4}\left(\theta_{1}, \theta_{2}\right)>0$ so that

and

$$
\frac{\left|\theta_{2}(x)-\theta_{2}(w)\right|}{\left|\theta_{2}(x)-\theta_{2}(z)\right|} \leq C_{1} \eta_{1}\left(\frac{\left|\theta_{1}(x)-\theta_{1}(w)\right|}{\left|\theta_{1}(x)-\theta_{1}(z)\right|}\right)
$$

$$
\frac{\left|\theta_{2}(x)-\theta_{2}(y)\right|}{\left|\theta_{2}(x)-\theta_{2}(w)\right|} \leq C_{4} \eta_{2}\left(\frac{\left|\theta_{1}(x)-\theta_{1}(y)\right|}{\left|\theta_{1}(x)-\theta_{1}(w)\right|}\right)
$$

where the homeomorphisms $\eta_{1}$ and $\eta_{2}$ are as in cases I and II.

Thus

$$
\begin{aligned}
\frac{\left|\theta_{2}(x)-\theta_{2}(y)\right|}{\left|\theta_{2}(x)-\theta_{2}(z)\right|} & =\frac{\left|\theta_{2}(x)-\theta_{2}(y)\right|}{\left|\theta_{2}(x)-\theta_{2}(w)\right|} \frac{\left|\theta_{2}(x)-\theta_{2}(w)\right|}{\left|\theta_{2}(x)-\theta_{2}(z)\right|} \\
& \leq C_{1} C_{4} \eta_{2}\left(\frac{\left|\theta_{1}(x)-\theta_{1}(y)\right|}{\left|\theta_{1}(x)-\theta_{1}(w)\right|}\right) \eta_{1}\left(\frac{\left|\theta_{1}(x)-\theta_{1}(w)\right|}{\left|\theta_{1}(x)-\theta_{1}(z)\right|}\right) \\
& \leq C_{1} C_{4} \eta_{2}\left(C \frac{\left|\theta_{1}(x)-\theta_{1}(y)\right|}{\left|\theta_{1}(x)-\theta_{1}(z)\right|}\right) \eta_{1}\left(C \frac{\left|\theta_{1}(x)-\theta_{1}(w)\right|}{\left|\theta_{1}(x)-\theta_{1}(z)\right|}\right),
\end{aligned}
$$

Thus (7.5) holds with $\eta=\eta_{3}$, where $\eta_{3}(t)=C_{1} C_{4} \eta_{1}(C t) \eta_{2}(C t)$.

Case III.3: The remaining case is that $x$ and $y$ are close. Let $H, H^{\prime} \in \mathcal{C}(\mathcal{X})$ be such that $\{x, y\} \subset \pi_{G}\left(H^{\text {diff }} \cup H^{\text {diff }}\right)$ and $H^{\text {diff }} \cap H^{\prime \text { diff }} \neq \emptyset$. As in Case III.2, there exist $C=C\left(\theta_{1}, \theta_{2}\right)>1$ and $w \in \pi_{G}\left(H^{\text {diff }} \cup H^{\prime \text { diff }}\right)$ so that

$$
\min \left\{\left|\theta_{i}(x)-\theta_{i}(w)\right|,\left|\theta_{i}(y)-\theta_{i}(w)\right|\right\} \geq \frac{1}{C} \operatorname{diam} \theta_{i}\left(\pi_{G}\left(H^{\text {diff }} \cup H^{\prime \text { diff }}\right)\right) .
$$

Furthermore, there exists $C_{5}=C_{5}\left(\theta_{1}, \theta_{2}\right)>0$ so that

$$
\frac{\left|\theta_{2}(x)-\theta_{2}(y)\right|}{\left|\theta_{2}(x)-\theta_{2}(z)\right|} \leq C_{5} \eta_{1}\left(\frac{\left|\theta_{1}(x)-\theta_{1}(y)\right|}{\left|\theta_{1}(x)-\theta_{1}(w)\right|}\right) \eta_{2}\left(\frac{\left|\theta_{1}(x)-\theta_{1}(w)\right|}{\left|\theta_{1}(x)-\theta_{1}(z)\right|}\right) .
$$

By (7.6) and the assumptions on $\{x, y, z\}$, we have that

$$
\max \left\{\frac{\left|\theta_{1}(x)-\theta_{1}(y)\right|}{\left|\theta_{1}(x)-\theta_{1}(z)\right|}, \frac{\left|\theta_{1}(x)-\theta_{1}(y)\right|}{\left|\theta_{1}(x)-\theta_{1}(w)\right|}, \frac{\left|\theta_{1}(x)-\theta_{1}(w)\right|}{\left|\theta_{1}(x)-\theta_{1}(z)\right|}\right\} \leq C^{\prime}
$$

where $C^{\prime}=C^{\prime}\left(\theta_{1}, \theta_{2}\right)$. Assume first that

$$
\frac{\left|\theta_{1}(x)-\theta_{1}(y)\right|}{\left|\theta_{1}(x)-\theta_{1}(w)\right|} \leq\left(\frac{\left|\theta_{1}(x)-\theta_{1}(y)\right|}{\left|\theta_{1}(x)-\theta_{1}(z)\right|}\right)^{1 / 2} .
$$

Then

$$
\frac{\left|\theta_{2}(x)-\theta_{2}(y)\right|}{\left|\theta_{2}(x)-\theta_{2}(z)\right|} \leq C_{5} \eta_{1}\left(\frac{\left|\theta_{1}(x)-\theta_{1}(y)\right|}{\left|\theta_{1}(x)-\theta_{1}(z)\right|}\right)^{1 / 2} \eta_{2}\left(C^{\prime}\right) .
$$


The case

$$
\frac{\left|\theta_{1}(x)-\theta_{1}(w)\right|}{\left|\theta_{1}(x)-\theta_{1}(z)\right|} \leq\left(\frac{\left|\theta_{1}(x)-\theta_{1}(y)\right|}{\left|\theta_{1}(x)-\theta_{1}(z)\right|}\right)^{1 / 2}
$$

is similar. So (7.5) holds with $\eta(t)=C_{5} \max \left\{\eta_{1}\left(t^{1 / 2}\right) \eta_{2}\left(C^{\prime}\right), \eta_{1}\left(C^{\prime}\right) \eta_{2}\left(t^{1 / 2}\right)\right\}$. This concludes consideration of Case III.2 and the proof.

\section{A sufficient condition for quasisymmetric parametrization}

In this section we consider the existence of a Semmes metric $d_{\lambda}$ on $\mathbb{R}^{3} / G$ such that $\left(\mathbb{R}^{3} / G, d_{\lambda}\right)$ is quasisymmetrically equivalent to $\mathbb{R}^{3}$. A sufficient condition for the parametrizability is the existence of a flat welding structure.

Definition 8.1. We say that $(\mathcal{C}, \mathcal{A}, \mathcal{W})$ is a flat welding structure if $\mathcal{C}$ is finite, condensers $\mathcal{C}$ are in $\mathbb{R}^{3}$, and weldings $\mathcal{W}$ are similarities.

The existence of a flat welding structure leads to a modular embedding $\theta$ of the decomposition space $\mathbb{R}^{3} / G$ into $\mathbb{R}^{4}$, which in turn shows a strong form of the quasisymmetric parametrizability of $\theta\left(\mathbb{R}^{3} / G\right)$, in particular, $\theta\left(\mathbb{R}^{3} / G\right)$ is a 3-dimensional quasiplane in $\mathbb{R}^{4}$.

Theorem 8.2. Suppose that $\left(\mathbb{R}^{3} / G, \mathcal{X}\right)$ is a decomposition space of finite type whose defining sequence $\mathcal{X}$ has a flat welding structure $(\mathcal{C}, \mathcal{A}, \mathcal{W})$. Suppose also that the components of $X_{k+1}$ are contractible in $X_{k}$ for every $k \geq 0$. Then there exists $\lambda_{0} \in(0,1)$ depending on $(\mathcal{C}, \mathcal{A}, \mathcal{W})$ satisfying the following conditions. For each $\lambda \in\left(0, \lambda_{0}\right)$, there is a $\lambda$-modular embedding $\theta: \mathbb{R}^{3} / G \rightarrow \mathbb{R}^{4}$ with respect to this structure, so that the embedded set $\theta\left(\mathbb{R}^{3} / G\right) \subset \mathbb{R}^{4}$ is Ahlfors 3-regular, linearly locally contractible, and quasisymmetric to $\mathbb{R}^{3}$. Furthermore, there exists a quasisymmetric map $f: \mathbb{R}^{4} \rightarrow \mathbb{R}^{4}$ so that $f\left(\mathbb{R}^{3}\right)=\theta\left(\mathbb{R}^{3} / G\right)$.

It is easy to see that every flat welding structure on a defining sequence of finite type induces a rigid welding structure with a compatible atlas, in the sense of (6.3). The converse is not always true; an obvious criterion can be given as follows.

Lemma 8.3. Let $\mathcal{X}$ be a defining sequence of finite type and let $(\mathcal{C}, \mathcal{A}, \mathcal{W})$ be a rigid welding structure on $\mathcal{X}$ in $\mathbb{R}^{3}$. Suppose that for every $\mathrm{c}=(A, B) \in \mathcal{C}$ there exists a $P L$ embedding $h_{\mathrm{c}}: A \rightarrow \mathbb{R}^{3}$ so that $h_{\mathrm{c}} \mid \partial A$ and $h_{\mathrm{c}} \mid D$ are (Euclidean) similarities for each component $D$ of $B$. Then $\mathcal{X}$ admits a flat welding structure.

All but the last claim in Theorem 8.2 can be proved by appealing to a rigid welding structure $\left(\mathcal{C}^{\prime}, \mathcal{A}^{\prime}, \mathcal{W}^{\prime}\right)$ compatible with the given flat structure $(\mathcal{C}, \mathcal{A}, \mathcal{W})$. However, in order to extend the quasisymmetric map $\mathbb{R}^{3} \rightarrow \theta\left(\mathbb{R}^{3} / G\right)$ to a quasisymmetric homeomorphism of $\mathbb{R}^{4}$, we will need to repack the condensers in $(\mathcal{C}, \mathcal{A}, \mathcal{W})$. The idea of repacking is adapted from Semmes's excellent packages for self-similar decomposition spaces ([19], Definition 3.2). 
Let $d_{\theta}$ be a $\lambda$-modular metric induced by an embedding $\theta: \mathbb{R}^{3} / G \rightarrow \mathbb{R}^{4}$ as in Theorem 8.2. By Lemma 8.3, the path metric $\hat{d}_{\theta}$ associated to $d_{\theta}$ is bilipschitz equivalent to the path metric associated to the Semmes metric (with the same scaling $\lambda$ ) derived from a compatible rigid structure $\left(\mathcal{C}^{\prime}, \mathcal{A}^{\prime}, \mathcal{W}^{\prime}\right)$. Thus $d_{\theta}$ is a Semmes metric and $\left(\mathbb{R}^{3} / G, \mathcal{X},(\mathcal{C}, \mathcal{A}, \mathcal{W}), \theta, d_{\theta}\right)$ a Semmes space; we write $d_{\lambda}$ for $d_{\theta}$.

In view of Theorem 8.2, there exist defining sequences which do not admit flat welding structures. Indeed, by Theorem 8.2, the existence of a flat welding structure yields quasisymmetric parametrizability. Thus, for example, the standard defining sequences associated to the Whitehead continuum and to the Bing double do not admit flat welding structures.

\subsection{Unlinking and repacking}

As a preliminary step for the proof of Theorem 8.2, we discuss homeomorphisms of $\mathbb{R}^{4}$ that unlink and repack condensers in $\mathbb{R}^{3}$.

Let $K \subset \mathbb{R}^{3}$ be a cube-with-handles. We define

$$
K^{*}=K \times[-\operatorname{diam} K, \operatorname{diam} K] \subset \mathbb{R}^{4},
$$

where diam $K$ is the Euclidean diameter of $K$. If $B$ is a pairwise disjoint union of cubes-with-handles, we set $B^{*}=\bigcup_{K \in \mathcal{C}(B)} K^{*}$. Suppose $(A, B)$ is a condenser in $\mathbb{R}^{3}$. We will also call $\left(A^{*}, B^{*}\right)$ a (4-dimensional) condenser.

Let $\mathrm{c}=(A, B)$ be a condenser in $\mathbb{R}^{3}$ with $\operatorname{diam} A=1$ and $\lambda \in(0,1)$. We say a PL-embedding $p_{\mathrm{c}}:\left(\mathbb{R}^{3} \backslash A\right) \cup B \rightarrow \mathbb{R}^{3}$ is a $\lambda$-repacking of $\mathrm{c}$ if there are pairwise disjoint Euclidean balls $\left\{b_{D} \subset \operatorname{int} A: D \in \mathcal{C}(B)\right\}$ such that

(i) $p_{\mathrm{c}} \mid \mathbb{R}^{3} \backslash A=\mathrm{id}$,

(ii) $p_{\mathrm{c}} \mid D$ is an orientation-preserving similarity, and

(iii) $p_{\mathrm{c}}(D) \subset$ int $b_{D}$ and $\operatorname{diam} p_{\mathrm{c}}(D)=\lambda$,

for each component $D$ of $B$.

Let $\mathrm{c}$ be a condenser in $\mathbb{R}^{3}$ with $\operatorname{diam} A=1$. We denote by $\lambda_{\mathrm{c}}$ the supremum of $\lambda>0$ so that $\mathrm{c}$ admits a $\lambda$-repacking. Note that $\lambda_{\mathrm{c}}>0$, since repackings exist for all sufficiently small $\lambda>0$.

Let $\mathcal{C}$ be a finite collection of condensers in $\mathbb{R}^{3}$, with $\operatorname{diam} A=1$ for every $\mathrm{c}=(A, B) \in \mathcal{C}$. We denote by $\lambda_{\mathcal{e}}$ the supremum of $\lambda>0$ so that every $\mathrm{c} \in \mathcal{C}$ admits a $\lambda$-repacking. We call $\lambda_{\mathcal{e}}$ the repacking constant of $\mathcal{C}$.

Definition 8.4. Let $\mathrm{c}=(A, B)$ be a condenser in $\mathbb{R}^{3}$ with $\operatorname{diam} A=1$. We say that a PL-homeomorphism $P_{\mathrm{c}}: \mathbb{R}^{4} \rightarrow \mathbb{R}^{4}$ is a ${ }^{*}$-stable $\lambda$-repacking of c in $\mathbb{R}^{3}$ (or of the condenser $\left.\left(A^{*}, B^{*}\right)\right)$ if

1) $P_{\mathrm{c}} \mid\left(\mathbb{R}^{3} \backslash A\right) \cup B$ is a $\lambda$-repacking of $\mathrm{c}$,

2) $P_{\mathrm{c}} \mid \mathbb{R}^{4} \backslash A^{*}=\mathrm{id}$,

3) $P_{\mathrm{c}} \mid D^{*}$ is an orientation preserving similarity for each component $D$ of $B$,

4) in particular, $P_{\mathrm{c}}\left(B^{*}\right)=P_{\mathrm{c}}(B)^{*}$. 
Lemma 8.5. Let $\mathrm{c}=(A, B)$ be a condenser in $\mathbb{R}^{3}$ with $\operatorname{diam} A=1$. If the components of $B$ are contractible in $A$, then, for every $0<\lambda<\lambda_{c}$, there exists a ${ }^{*}$-stable $\lambda$-repacking $P_{\mathrm{c}}: \mathbb{R}^{4} \rightarrow \mathbb{R}^{4}$ of $\mathrm{c}$.

Proof. Let $p_{\mathrm{c}}:\left(\mathbb{R}^{3} \backslash A\right) \cup B \rightarrow \mathbb{R}^{3}$ be a $\lambda$-repacking of the condenser $\mathrm{c}=(A, B)$. Fix $d \in(0,1)$ so that

$$
B^{*} \cup\left(p_{\mathrm{c}}(B)\right)^{*} \subset \operatorname{int}(A \times[-d, d]),
$$

and set $I=[-d, d]$.

As a preliminary step, we construct for every $D \in \mathcal{C}(B)$ a PL-homeomorphism $f_{D}: \mathbb{R}^{4} \rightarrow \mathbb{R}^{4}$ with the properties that $f_{D} \mid \mathbb{R}^{4} \backslash(A \times I)=$ id and that $f_{D} \mid D^{*}$ is an orientation-preserving similarity satisfying $f_{D}\left|D=p_{\mathrm{c}}\right| D$ and $f_{D}\left(D^{*}\right)=p_{\mathrm{c}}(D)^{*}$.

Given $D \in \mathcal{C}(B)$, let $b_{D}=B^{3}\left(x_{D}, r_{D}\right) \subset A$ be the Euclidean ball containing $p_{\mathrm{c}}(D)$ as in (iii). Since $p_{\mathrm{c}} \mid D$ is a similarity, it extends to a similarity $p_{D}: \mathbb{R}^{3} \rightarrow \mathbb{R}^{3}$ with a scaling constant $\rho_{D}$. Denote again by $p_{D}: \mathbb{R}^{4} \rightarrow \mathbb{R}^{4}$ the extension $(x, t) \mapsto$ $\left(p_{D}(x), \rho_{D} t\right)$.

Fix a core $\mathcal{R}_{D}$ of $D$. Since $D^{*}$ is a regular neighborhood of $\mathcal{R}_{D}$ and $\mathcal{R}_{D}$ is contractible in $A$, there exist, by the Penrose-Whitehead-Zeeman lemma (see Section 3.2), PL 4-cells $E_{D}$ and $E_{D}^{\prime}$ in $A \times I$ so that

$$
D^{*} \subset \operatorname{int} E_{D} \subset E_{D} \subset \operatorname{int} E_{D}^{\prime} \subset E_{D}^{\prime} \subset \operatorname{int}(A \times I) .
$$

We fix $z_{D} \in \operatorname{int} E_{D}$ and $\varepsilon_{D}>0$ so that $B^{4}\left(z_{D}, 2 \varepsilon_{D}\right) \subset E_{D}$, and choose a number $r_{D}^{\prime} \in\left(r_{D}, \lambda_{c}\right)$. Thus

$$
p_{D}\left(D^{*}\right) \subset b_{D}^{*} \subset B^{3}\left(x_{D}, r_{D}^{\prime}\right)^{*} \subset \operatorname{int}(A \times I) .
$$

By standard isotopy results, we may fix two PL self-homeomorphisms $h_{D}$ and $\tau_{D}$ of $\mathbb{R}^{4}$ with the following properties. Since $E_{D}$ and $E_{D}^{\prime}$ are 4 -cells, there exists an orientation-preserving PL homeomorphism $h_{D}: \mathbb{R}^{4} \rightarrow \mathbb{R}^{4}$ so that $h_{D} \mid \mathbb{R}^{4} \backslash$ $(A \times I)=\mathrm{id}, h_{D}\left(E_{D}^{\prime}\right)=B^{4}\left(z_{D}, 2 \varepsilon_{D}\right), h_{D}\left(E_{D}\right)=B^{4}\left(z_{D}, \varepsilon_{D}\right)$, and $h_{D}\left(D^{*}\right) \subset$ $B^{4}\left(z_{D}, \varepsilon_{D} / 2\right)$, and so that $h_{D} \mid D^{*}$ is a scaling followed by a translation. Furthermore, there exists a PL-homeomorphism $\tau_{D}: \mathbb{R}^{4} \rightarrow \mathbb{R}^{4}$ such that $\tau_{D} \mid \mathbb{R}^{4} \backslash(A \times I)=$ id, $\tau_{D}\left(B^{4}\left(z_{D}, 2 \varepsilon_{D}\right)\right)=B^{3}\left(x_{D}, r_{D}^{\prime}\right)^{*}$, and $\tau_{D}\left(B^{4}\left(z_{D}, \varepsilon_{D}\right)\right)=b_{D}^{*}$, and so that $\tau_{D} \mid h_{D}\left(D^{*}\right)$ is an orientation preserving similarity that maps $h_{D}\left(D^{*}\right)$ onto $p_{D}\left(D^{*}\right)$.

Therefore, the PL homeomorphism $f_{D}=\tau_{D} \circ h_{D}$ satisfies $f_{D} \mid \mathbb{R}^{4} \backslash(A \times I)=\mathrm{id}$ and $f_{D} \mid D^{*}$ is the similarity $p_{D} \mid D^{*}$.

We will combine the homeomorphisms $f_{D}, D \in \mathcal{C}(B)$, defined above as follows. First, the components of $B$ are raised to different levels in $\mathbb{R}^{3} \times(d, 1) \subset \mathbb{R}^{4}$ by a homeomorphism $g_{1}$ of $\mathbb{R}^{4}$. Next, one component at a time, each $D \in \mathcal{C}(B)$ is lowered to $\left\{x_{4}=0\right\}$, where the homeomorphism $f_{D}$ may be applied, and then the image $p_{D}(D)$ is raised to the previous level. The composition of these maps is a homeomorphism $g_{2}$ of $\mathbb{R}^{4}$. Finally, all raised $p_{D}(D)$ are descended to $\left\{x_{4}=0\right\}$ by a homeomorphism $g_{3}^{-1}$ of $\mathbb{R}^{4}$. The ${ }^{*}$-stable $\lambda$-repacking of $\mathrm{c}$ is defined by

$$
P_{\mathrm{c}}=g_{3}^{-1} \circ g_{2} \circ g_{1}
$$


We now give the details.

For every $D \in \mathcal{C}(B)$, fix $d_{D} \in(d, 1)$ so that $d_{D} \neq d_{D^{\prime}}$ for different components $D$ and $D^{\prime}$ in $\mathcal{C}(B)$; fix also $\delta>0$ so that the intervals $\left[d_{D}-\delta, d_{D}+\delta\right]$ are pairwise disjoint and contained in $(d, 1)$. Let $\rho=\delta /(4 d)$ and set $J_{D}=\left[d_{D}-\delta / 4, d_{D}+\delta / 4\right]$ for every $D \in \mathcal{C}(B)$. We fix a PL-homeomorphism $g_{1}: \mathbb{R}^{3} \times \mathbb{R} \rightarrow \mathbb{R}^{3} \times \mathbb{R}$ so that $g_{1} \mid \mathbb{R}^{4} \backslash A^{*}=$ id and $g_{1}(x, t)=\left(x, \rho t+d_{D}\right)$ for $(x, t) \in D \times I$ and $D \in \mathcal{C}(B)$. In particular,

$$
g_{1}\left(D^{*}\right) \subset g_{1}(D \times I)=D \times J_{D}
$$

for every $D \in \mathcal{C}(B)$.

The homeomorphism $g_{3}$ is defined similarly as $g_{1}$, with $\left(A, \bigcup_{D \in \mathcal{C}(B)} p_{D}(D)\right)$ in place of $(A, B)$ and with $d_{p_{D}(D)}=d_{D}$, so that the PL-homeomorphism $g_{3}: \mathbb{R}^{4} \rightarrow \mathbb{R}^{4}$ satisfies $g_{3} \mid \mathbb{R}^{4} \backslash A^{*}=\mathrm{id}$ and

$$
g_{3}\left(p_{D}(D)^{*}\right) \subset g_{3}\left(p_{D}(D) \times I\right) \subset p_{D}(D) \times J_{D}
$$

for $D \in \mathcal{C}(B)$.

Having $g_{1}$ and $g_{3}$ at our disposal, we construct a PL-homeomorphism $g_{2}$ as follows. For every $D \in \mathcal{C}(B)$, let $\zeta_{D}: \mathbb{R} \rightarrow \mathbb{R}$, be a piecewise linear increasing function so that $\zeta_{D}(t)=\rho t+d_{D}$ for $t \in I$, and $\zeta_{D}(t)=t$ for $|t|>1$. Let also $\xi_{D}: \mathbb{R}^{4} \rightarrow \mathbb{R}^{4}$ be the PL map $(x, t) \mapsto\left(x, \zeta_{D}(t)\right)$. Then $\xi_{D}\left|D^{*}=g_{1}\right| D^{*}$ and $\xi_{D}\left|p_{D}(D)^{*}=g_{3}\right| p_{D}(D)^{*}$ for every $D \in \mathcal{C}(B)$.

Since $f_{D} \mid \mathbb{R}^{4} \backslash(A \times I)=\mathrm{id}$, we have

$$
\xi_{D} \circ f_{D} \circ \xi_{D}^{-1} \mid\left(\mathbb{R}^{4} \backslash\left(A \times\left(d_{D}-\delta / 4, d_{D}+\delta / 4\right)\right)\right)=\mathrm{id}
$$

for every $D \in \mathcal{C}(B)$. Thus the mapping $g_{2}: \mathbb{R}^{4} \rightarrow \mathbb{R}^{4}$ defined by taking the composition (in any fixed order) of $\xi_{D} \circ f_{D} \circ \xi_{D}^{-1}$ for all $D \in \mathcal{C}(B)$, is a well-defined PL-homeomorphism satisfying $g_{2} \mid \mathbb{R}^{4} \backslash A^{*}=\mathrm{id}$. Moreover,

$$
g_{3}^{-1} \circ g_{2} \circ g_{1}\left|D^{*}=\left(g_{3}^{-1} \circ \xi_{D}\right) \circ f_{D} \circ\left(\xi_{D}^{-1} \circ g_{1}\right)\right| D^{*}=f_{D}\left|D^{*}=p_{D}\right| D^{*}
$$

is a similarity. Since $p_{\mathrm{c}}\left|D=p_{D}\right| D, P_{\mathrm{c}}=g_{3}^{-1} \circ g_{2} \circ g_{1}$ is a $*_{\text {-stable repacking }}$ of $\mathrm{c}$.

\subsection{Proof of Theorem 8.2}

Let $(\mathcal{C}, \mathcal{A}, \mathcal{W})$ be a flat welding structure on the defining sequence $\mathcal{X}$, and assume that $\operatorname{diam} A=1$ for all $\mathrm{c}=(A, B) \in \mathcal{C}$. We also assume that $X_{0}=H_{0}=A_{H_{0}}$, where $\mathrm{c}_{H_{0}}=\left(A_{H_{0}}, B_{H_{0}}\right)$ is the condenser associated to $H_{0}$, and that the corresponding chart satisfies $\varphi_{H_{0}} \mid \partial H_{0}=\mathrm{id}$.

We enumerate the cubes-with-handles in $\mathcal{C}(\mathcal{X})$ by $H_{0}, H_{1}, \ldots$ so that if $H_{j} \in$ $\mathcal{C}\left(X_{k}\right)$ then $H_{j+1} \in \mathcal{C}\left(X_{k}\right) \cup \mathcal{C}\left(X_{k+1}\right)$. This enumeration induces natural orderings on condensers, charts, and weldings as well. Write $\mathrm{c}_{j}=\left(A_{j}, B_{j}\right)=\mathrm{c}_{H_{j}}$ for condensers in $\mathcal{C}$ and $\varphi_{j}=\varphi_{H_{j}}: H_{j}^{\text {diff }} \rightarrow \mathrm{c}_{j}^{\text {diff }}$ for the charts in $\mathcal{A}$ for $j \geq 0$.

Let $k_{j}=$ level $\left(H_{j}\right)$, and let $q(j)$ be the index of the parent of $H_{j}$, that is, level $\left(H_{q(j)}\right)=k_{j}-1$ and $H_{j} \in \mathcal{C}\left(H_{q(j)} \cap X_{k_{j}}\right)$. 
Let $w_{j}=\varphi_{q(j)} \circ \varphi_{j}^{-1}$ be the welding of $\left(A_{j}, B_{j}\right)$ to its parent $\left(A_{q(j)}, B_{q(j)}\right)$, for $j \geq 1$. Since $w_{j}$ is a similarity and $B_{q(j)} \subset \mathbb{R}^{3}, w_{j}\left(A_{j}\right)$ is a component of $B_{q(j)}$. We extend $w_{j}$ to a similarity $w_{j}: \mathbb{R}^{4} \rightarrow \mathbb{R}^{4}$ by $(x, t) \mapsto\left(w_{j}(x), \lambda_{j} t\right)$, where $\lambda_{j}$ is the scaling factor of $w_{j}$. We call the extended $w_{j}$ a welding of $\left(A_{j}^{*}, B_{j}^{*}\right)$ to $\left(A_{q(j)}^{*}, B_{q(j)}^{*}\right)$, and note that $w_{j}\left(A_{j}^{*}\right)=w_{j}\left(A_{j}\right)^{*}$ is a component of $B_{q(j)}^{*}$.

We construct now cumulative welding maps and repacked cumulative welding maps. We define the cumulative welding maps $\hat{w}_{j}$ by $\hat{w}_{0}=\mathrm{id}$ and

$$
\hat{w}_{j}=\hat{w}_{q(j)} \circ w_{j}
$$

for $j \geq 1$.

Since $w_{j}\left|\partial A_{j}=\varphi_{q(j)} \circ \varphi_{j}^{-1}\right| \partial A_{j}$, we have

$$
\hat{w}_{j} \circ \varphi_{j}\left|\partial H_{j}=\hat{w}_{q(j)} \circ w_{j} \circ \varphi_{j}\right| \partial H_{j}=\hat{w}_{q(j)} \circ \varphi_{q(j)} \mid \partial H_{j}
$$

for $j \geq 1$, and $\hat{w}_{0} \circ \varphi_{0} \mid \partial H_{0}=\mathrm{id}$.

Since $w_{j}$ is a similarity, $\hat{w}_{j}\left(A_{j}\right)$ is a component of $\hat{w}_{q(j)}\left(B_{q(j)}\right)$ and

$$
\hat{w}_{j}\left(A_{j}^{*}\right)=\hat{w}_{q(j)}\left(w_{j}\left(A_{j}^{*}\right)\right) \subset \hat{w}_{q(j)}\left(B_{q(j)}^{*}\right) .
$$

It follows by induction that the images $\hat{w}_{j}\left(A_{j}^{*} \backslash B_{j}^{*}\right)$ are pairwise disjoint for $j \geq 0$. Then

$$
\mathbb{R}^{4} \backslash \hat{F}=\left(\mathbb{R}^{4} \backslash X_{0}^{*}\right) \bigcup \bigcup_{j=1}^{\infty} \hat{w}_{j}\left(A_{j}^{*} \backslash B_{j}^{*}\right)
$$

is a disjoint union, where

$$
\hat{F}=\bigcap_{k \geq 0}\left(\bigcup\left\{\hat{w}_{j}\left(A_{j}\right):\left(A_{j}, B_{j}\right) \in \mathcal{C}, \varphi_{j}^{-1}\left(A_{j}\right)=H_{j} \in \mathcal{C}\left(X_{k}\right)\right\}\right) .
$$

Since diam $\hat{w}_{j}\left(A_{j}\right) \rightarrow 0$ as $j \rightarrow \infty$, the components of $\hat{F}$ are points.

Now we define repacked cumulative welding maps $\tilde{w}_{j}$. Let $0<\lambda<\lambda_{\mathcal{e}}$. We show first that the components of $B_{j}$ are contractible in $A_{j}$. Let $D \in \mathcal{C}\left(B_{j}\right)$. Since $\varphi_{j}^{-1}(\partial D)$ is the boundary of a component of $H_{j} \cap X_{\text {level }\left(H_{j}\right)+1}, \varphi_{j}^{-1}(\partial D)$ is contractible in $H_{j}$. Thus $\partial D$ is contractible in $A_{j}$. Let $\mathcal{R}_{D}$ be a core of $D$ that is contained in a collar $\Omega_{D}$ of $\partial D$ in $D$. Since $\Omega_{D}$ retracts to $\partial D, \mathcal{R}_{D}$ is contractible in $A_{j}$. Thus $D$ is contractible in $A_{j}$.

Using Lemma 8.5 , we fix a collection of ${ }^{*}$-stable $\lambda$-repackings $\left\{P_{\mathrm{c}}: \mathbb{R}^{4} \rightarrow\right.$ $\left.\mathbb{R}^{4}: c \in \mathcal{C}\right\}$. For simplicity, denote the ${ }^{*}$-stable repacking for $\mathrm{c}_{j}=\left(A_{j}, B_{j}\right)$ by $P_{j}=P_{\mathrm{c}_{j}}$ for $j \geq 0$; note that there are only finitely many distinct mappings in $\left\{P_{j}: j \geq 0\right\}$.

Associated to the welding maps $w_{j}: \mathbb{R}^{4} \rightarrow \mathbb{R}^{4}$ for $j \geq 1$, and the ${ }^{*}$-stable repackings $P_{j}$ for $j \geq 0$, we define $\tilde{w}_{j}: \mathbb{R}^{4} \rightarrow \mathbb{R}^{4}$ by

$$
\tilde{w}_{j}=\tilde{w}_{q(j)} \circ w_{j} \circ P_{j}
$$

for $j \geq 1$ and set $\tilde{w}_{0}=P_{0}$. 
Since the ${ }^{*}$-stable repacking $P_{j}$ is a similarity on $D^{*}$ for each $D \in \mathcal{C}\left(B_{j}\right)$ and $P_{j}\left(A_{j}^{*}\right)=A_{j}^{*}$, we know that $w_{j} \circ P_{j}\left(A_{j}^{*}\right) \subset B_{q(j)}^{*}$ and that $\tilde{w}_{j} \mid D^{*}$ is a similarity for every $D \in \mathcal{C}\left(B_{j}\right)$. Therefore $\tilde{w}_{q(j)} \circ w_{j} \mid A_{j}^{*}$ is a similarity, and $\tilde{w}_{j} \mid A_{j}^{*} \backslash B_{j}^{*}$ is a composition of an $L$-bilipschitz map $P_{j}$ with a similarity for every $j \geq 0$. Indeed, the mapping $\tilde{w}_{j} \mid A_{j}$ is $L \lambda^{j}$-Lipschitz for every $j \geq 0$, where $L$ is the maximum of

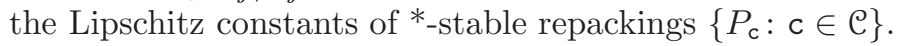

Since $P_{j} \mid \partial A_{j}=\mathrm{id}$, we have, as in (8.1),

$$
\tilde{w}_{j} \circ \varphi_{j}\left|\partial H_{j}=\tilde{w}_{q(j)} \circ \varphi_{q(j)}\right| \partial H_{j}
$$

for $j \geq 1$, and $\tilde{w}_{0} \circ \varphi_{0}\left|\partial H_{0}=P_{0} \circ \varphi_{0}\right| \partial H_{0}=\mathrm{id}$.

Since $\tilde{w}_{j}\left(A_{j}\right)$ is a component of $\tilde{w}_{q(j)}\left(B_{q(j)}\right)$ for each $j \geq 1$ and the image sets $\tilde{w}_{j}\left(A_{j}^{*} \backslash B_{j}^{*}\right)$ are pairwise disjoint for $j \geq 0$, we obtain a disjoint union

$$
\mathbb{R}^{4} \backslash \tilde{F}=\left(\mathbb{R}^{4} \backslash X_{0}^{*}\right) \bigcup \bigcup_{j=1}^{\infty} \tilde{w}_{j}\left(A_{j}^{*} \backslash B_{j}^{*}\right)
$$

with

$$
\tilde{F}=\bigcap_{k \geq 0}\left(\bigcup\left\{\tilde{w}_{j}\left(A_{j}\right):\left(A_{j}, B_{j}\right) \in \mathcal{C}, \varphi_{j}^{-1}\left(A_{j}\right)=H_{j} \in \mathcal{C}\left(X_{k}\right)\right\}\right) .
$$

As in the case of $\hat{F}$, the set $\tilde{F}$ is totally disconnected.

Having (8.3) and (8.4) at our disposal, we define an embedding $\theta_{\infty}: \mathbb{R}^{3} \backslash X_{\infty} \rightarrow$ $\mathbb{R}^{4}$ by $\theta_{\infty} \mid \mathbb{R}^{3} \backslash X_{0}=$ id and $\theta_{\infty} \mid H_{j}^{\text {diff }}=\tilde{w}_{j} \circ \varphi_{j}$ for $j \geq 1$. Furthermore, $\theta_{\infty}$ descends (and then extends) to an embedding $\theta: \mathbb{R}^{3} / G \rightarrow \mathbb{R}^{4}$ so that $\theta\left(\pi_{G}\left(X_{\infty}\right)\right)=\tilde{F}$. The $\lambda$-modularity of $\theta$ with respect to $(\mathcal{C}, \mathcal{A}, \mathcal{W})$ follows directly from the uniform quasisimilarity of the cumulative repacked welding maps $\tilde{w}_{j}$. The space $\left(\mathbb{R}^{3} / G, \mathcal{X},(\mathcal{C}, \mathcal{A}, \mathcal{W}), \theta, d_{\theta}\right)$ is linearly locally connected and Ahlfors 3-regular for sufficiently small $\lambda$.

It remains to construct a quasisymmetric map $f: \mathbb{R}^{4} \rightarrow \mathbb{R}^{4}$ so that $f\left(\mathbb{R}^{3}\right)=$ $\theta\left(\mathbb{R}^{3} / G\right)$. Since $P_{j} \mid \partial A_{j}^{*}=\mathrm{id}$, we have

$\tilde{w}_{j} \circ \hat{w}_{j}^{-1}\left|\hat{w}_{j}\left(\partial A_{j}^{*}\right)=\left(\tilde{w}_{q(j)} \circ w_{j} \circ P_{j}\right) \circ\left(w_{j}^{-1} \circ \hat{w}_{q(j)}^{-1}\right)\right| \hat{w}_{j}\left(\partial A_{j}^{*}\right)=\tilde{w}_{q(j)} \circ \hat{w}_{q(j)}^{-1} \mid \hat{w}_{j}\left(\partial A_{j}^{*}\right)$

for every $j \geq 1$. Thus the map $f_{\infty}: \mathbb{R}^{4} \backslash \hat{F} \rightarrow \mathbb{R}^{4} \backslash \tilde{F}$, defined by

$$
f_{\infty}\left|\hat{w}_{j}\left(A_{j}^{*} \backslash B_{j}^{*}\right)=\tilde{w}_{j} \circ \hat{w}_{j}^{-1}\right| \hat{w}_{j}\left(A_{j}^{*} \backslash B_{j}^{*}\right)
$$

for $j \geq 1$ and $f_{\infty} \mid \mathbb{R}^{4} \backslash A_{0}^{*}=\mathrm{id}$, is a well-defined homeomorphism. Since $\hat{F}$ and $\tilde{F}$ are totally disconnected, $f_{\infty}$ extends to a homeomorphism $f: \mathbb{R}^{4} \rightarrow \mathbb{R}^{4}$.

Since $f\left|\mathbb{R}^{3} \backslash A_{0}=\theta_{\infty}\right| \mathbb{R}^{3} \backslash X_{0}$ and

$$
f \circ \hat{w}_{j} \circ \varphi_{j}\left|X_{j}^{\text {diff }}=\tilde{w}_{j} \circ \hat{w}_{j}^{-1} \circ \hat{w}_{j} \circ \varphi_{j}\right| X_{j}^{\text {diff }}=\tilde{w}_{j} \circ \varphi_{j}\left|X_{j}^{\text {diff }}=\theta_{\infty}\right| X_{j}^{\text {diff }}
$$

for every $j \geq 0$, we have

$$
f\left(\mathbb{R}^{3}\right)=\theta\left(\mathbb{R}^{3} / G\right)
$$

by continuity. 
Since $\tilde{w}_{j} \circ \hat{w}_{j}^{-1} \mid \hat{w}_{j}\left(A_{j}^{*}\right)$ is a $\left(L, \mu_{j}\right)$-quasisimilarity for some $\mu_{j}>0$ and for every $j \geq 0$, the homeomorphism $f_{\infty}: \mathbb{R}^{4} \backslash \hat{F} \rightarrow \mathbb{R}^{4} \backslash \tilde{F}$ is quasiconformal. Moreover, the homeomorphisms $f_{j}: \mathbb{R}^{4} \rightarrow \mathbb{R}^{4}$, defined by

$$
f_{j}\left|\mathbb{R}^{4} \backslash \hat{w}_{j}\left(A_{j}^{*}\right)=f_{\infty}\right| \mathbb{R}^{4} \backslash \hat{w}_{j}\left(A_{j}^{*}\right)
$$

and

$$
f_{j}\left|\hat{w}_{j}\left(A_{j}^{*}\right)=\tilde{w}_{j} \circ \hat{w}_{j}^{-1}\right| \hat{w}_{j}\left(A_{j}^{*}\right),
$$

are uniformly quasiconformal. Therefore there exists a homeomorphism $\eta:[0, \infty) \rightarrow$ $[0, \infty)$ so that the homeomorphisms $f_{j}$ are $\eta$-quasisymmetric. Since $f=\lim _{j \rightarrow \infty} f_{j}$, $f$ is $\eta$-quasisymmetric. This completes the proof of Theorem 8.2.

Remark 8.6. The quasisymmetric map $f: \mathbb{R}^{4} \rightarrow \mathbb{R}^{4}$ in Theorem 8.2 can be taken to be the identity in a neighborhood of infinity, that is, there exists $R>0$ so that $f \mid \mathbb{R}^{4} \backslash B^{4}(R)=\mathrm{id}$. Thus the quasisymmetric map $f: \mathbb{R}^{4} \rightarrow \mathbb{R}^{4}$ extends naturally to a quasiconformal map $f: \mathbb{S}^{4} \rightarrow \mathbb{S}^{4}$ and $f\left(\mathbb{S}^{3}\right)$ is the one point compactification of $f\left(\mathbb{R}^{3}\right)$. Thus the embedding $\theta: \mathbb{R}^{3} / G \rightarrow \mathbb{R}^{4}$ extends to an embed$\operatorname{ding} \mathbb{S}^{3} / G \rightarrow \mathbb{S}^{4}$. So $\theta\left(\mathbb{S}^{3} / G\right)$ is a quasisphere, that is, $\theta\left(\mathbb{S}^{3} / G\right)=f\left(\mathbb{S}^{3}\right)$, where $f: \mathbb{S}^{4} \rightarrow \mathbb{S}^{4}$ is a quasiconformal map.

In view of Theorem 8.2, geometrically different quasispheres built this way exist in abundance.

\section{Circulation}

In this section we introduce the notion of the circulation of a union of cubes-withhandles based on longitudes and meridians. This concept of circulation will be used in estimating the conformal moduli of surface families.

\subsection{Meridians and longitudes}

Recall that a simple closed curve $\mathbb{S}^{1} \rightarrow \partial \mathbb{B}^{2} \times \mathbb{S}^{1}$ on the boundary of a torus $\mathbb{B}^{2} \times \mathbb{S}^{1}$ is called a meridian of $\mathbb{B}^{2} \times \mathbb{S}^{1}$ if it is homotopic to the loop $e^{i \theta} \mapsto\left(e^{i \theta}, 1\right)$, on $\partial \mathbb{B}^{2} \times \mathbb{S}^{1}$. In particular, a meridian is contractible in $\mathbb{B}^{2} \times \mathbb{S}^{1}$ but not in $\partial \mathbb{B}^{2} \times \mathbb{S}^{1}$.

A noncontractible loop in the solid torus $\mathbb{B}^{2} \times \mathbb{S}^{1}$ is called a longitude of $\mathbb{B}^{2} \times \mathbb{S}^{1}$. A longitude is nontrivially linked with every meridian, that is, given a longitude $\sigma$ and a meridian $\alpha$ of $\mathbb{B}^{2} \times \mathbb{S}^{1}$ then $\sigma\left(\mathbb{S}^{1}\right) \cap \phi\left(\mathbb{B}^{2}\right) \neq \emptyset$ for every $\phi: \mathbb{B}^{2} \rightarrow \mathbb{B}^{2} \times \mathbb{S}^{1}$ satisfying $\phi \mid \partial \mathbb{B}^{2}=\alpha$.

Let $X$ be a disjoint union of cubes-with-handles. We call a simple closed PL curve $\alpha: \mathbb{S}^{1} \rightarrow \partial X$ a meridian of $X$ if $[\alpha] \neq 0$ in $\pi_{1}(\partial X)$ and $[\alpha]=0$ in $\pi_{1}(X)$; that is, $\alpha$ is not contractible on $\partial X$ but there exists a map $\phi: \mathbb{B}^{2} \rightarrow X$ so that $\phi \mid \partial \mathbb{B}^{2}=\alpha$.

Suppose $\alpha: \mathbb{S}^{1} \rightarrow \partial X$ is a meridian of $X$. Departing slightly from the standard notion of a mapping of pair $(C, D) \rightarrow(E, F)$, we denote by $\phi:\left(\mathbb{B}^{2}, \partial \mathbb{B}^{2}\right) \rightarrow(X, \alpha)$ a mapping $\phi: \mathbb{B}^{2} \rightarrow X$ that satisfies $\phi \mid \partial \mathbb{B}^{2}=\alpha$. Let

$$
\mathcal{E}(X, \alpha)=\text { the collection of all maps } \phi:\left(\mathbb{B}^{2}, \partial \mathbb{B}^{2}\right) \rightarrow(X, \alpha) .
$$


Let $\sigma=\sigma_{1}+\cdots+\sigma_{k}$ be a PL 1-chain in a union of cubes-with-handles $X$, where $\sigma_{i}: \mathbb{S}^{1} \rightarrow X$ are PL maps for $i=1, \ldots, k$; and let $|\sigma|=\bigcup_{i=1}^{k} \sigma_{i}\left(\mathbb{S}^{1}\right)$ be its carrier. We say that $\sigma$ is a longitude in $X$ if $|\sigma| \cap \phi\left(\mathbb{B}^{2}\right) \neq \emptyset$ for all $\phi:\left(\mathbb{B}^{2}, \partial \mathbb{B}^{2}\right) \rightarrow(X, \alpha)$ and all meridians $\alpha$ of $X$. Heuristically, a longitude is a 1-cycle in $X$ that is linked with every meridian of $X$. We let

$$
\Sigma(X)=\text { the family of all longitudes of } X \text {. }
$$

Suppose that $H_{1}, \ldots, H_{d}$ are pairwise disjoint cubes-with-handles, then

$$
\Sigma\left(\bigcup_{i=1}^{d} H_{i}\right)=\left\{\sigma_{1}+\cdots+\sigma_{d}: \sigma_{i} \in \Sigma\left(H_{i}\right), 1 \leq i \leq d\right\} .
$$

\subsection{Circulation with respect to meridians}

Let $H$ be a cube-with-handles, let $X$ be a finite union of cubes-with-handles in int $H$, and let $\alpha: \mathbb{S}^{1} \rightarrow \partial H$ be a meridian of $H$. The circulation of $X$ in $H$ with respect to the meridian $\alpha$ is defined to be

$$
\operatorname{circ}(X, \alpha, H)=\min _{\phi \in \mathcal{E}(H, \alpha)} \min _{\sigma \in \Sigma(X)} \#\left(|\sigma| \cap \phi\left(\mathbb{B}^{2}\right)\right) .
$$
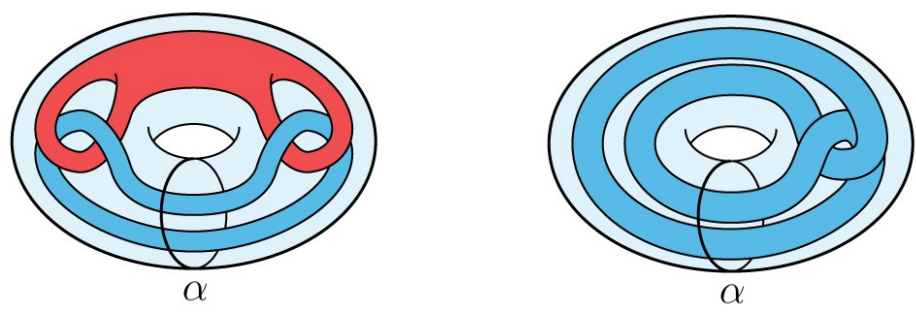

FiguRE 7. Circulations having values 1 and 2 with respect to $\alpha$.

Let $\left(\mathbb{R}^{3} / G, \mathcal{X},(\mathcal{C}, \mathcal{A}, \mathcal{W})\right)$ be a decomposition space of finite type. We call a meridian of any cube-with-handles in $\mathcal{X}$ a meridian of $\mathcal{X}$. We denote this family of meridians by $\mathcal{M}(\mathcal{X})$.

Definition 9.1. Let $\left(\mathbb{R}^{3} / G, \mathcal{X},(\mathcal{C}, \mathcal{A}, \mathcal{W})\right)$ be a decomposition space of finite type. Meridians $\alpha: \mathbb{S}^{1} \rightarrow \partial H$ and $\alpha^{\prime}: \mathbb{S}^{1} \rightarrow \partial H^{\prime}$ in $\mathcal{M}(\mathcal{X})$ are related by the atlas $\mathcal{A}$ if the charts $\varphi_{H}$ and $\varphi_{H^{\prime}}$ have the same target condenser in $\mathcal{C}$ and $\varphi_{H}^{-1} \circ \varphi_{H^{\prime}} \circ \alpha^{\prime}$ is homotopic to $\alpha$ on $\partial H$.

Given a meridian $\alpha$ in $\mathcal{X}$,

$$
\mathcal{M}_{\mathcal{A}}(\mathcal{X} ; \alpha)=\left\{\alpha^{\prime} \in \mathcal{M}(\mathcal{X}): \alpha \text { and } \alpha^{\prime} \text { are related by atlas } \mathcal{A}\right\}
$$

is called the set of meridians in $\mathcal{X}$ related to $\alpha$ by $\mathcal{A}$. 
Definition 9.2. Let $\left(\mathbb{R}^{3} / G, \mathcal{X},(\mathcal{C}, \mathcal{A}, \mathcal{W})\right)$ be a decomposition space of finite type. We say that the order of circulation of $\mathcal{X}$ is at least $\omega, \omega \geq 0$, if there exist a meridian $\alpha_{0} \in \mathcal{M}(\mathcal{X})$ and a constant $C>0$ such that for every $\ell \geq 0$ there exist $k^{\prime}>k \geq 0$ with $k^{\prime}-k>\ell$, a cube-with-handles $H \in \mathcal{C}\left(X_{k}\right)$, and a meridian $\alpha: \mathbb{S}^{1} \rightarrow \partial H$ in $\mathcal{M}_{\mathcal{A}}\left(\mathcal{X} ; \alpha_{0}\right)$ that satisfy

$$
\operatorname{circ}\left(X_{k^{\prime}} \cap H, \alpha, H\right) \geq C \omega^{k^{\prime}-k} .
$$

The homotopy property of a collection of meridians translates to geometric finiteness in the corresponding Semmes space after fixing a simple PL-representative for each homotopy class.

Lemma 9.3. Let $\left(\mathbb{R}^{3} / G, \mathcal{X},(\mathcal{C}, \mathcal{A}, \mathcal{W}), \theta, d_{\lambda}\right)$ be a Semmes space and let $\alpha_{0} \in$ $\mathcal{M}(\mathcal{X})$. Then there exists $L=L\left(d_{\lambda}, \alpha_{0}\right) \geq 1$ so that for every meridian $\alpha: \mathbb{S}^{1} \rightarrow$ $\partial H$ in $\mathcal{M}_{\mathcal{A}}\left(\mathcal{X} ; \alpha_{0}\right)$ there exists a meridian $\beta$ of $H$ homotopic to $\alpha$ in $\partial H$ so that $\pi_{G} \circ \beta: \mathbb{S}^{1} \rightarrow\left(\mathbb{R}^{3} / G, d_{\lambda}\right)$ is a $\left(\lambda^{\operatorname{level}(H)}, L\right)$-quasisimilarity.

We record the observation that a quasisimilar meridian has a quasisimilar collar. The claim follows directly from properties of the metric $d_{\lambda, m}$ and the definition of the constant $\epsilon_{\lambda}$ in Remark 7.7.

Lemma 9.4. Let $\left(\mathbb{R}^{3} / G, \mathcal{X},(\mathcal{C}, \mathcal{A}, \mathcal{W}), \theta, d_{\lambda}\right)$ be a Semmes space and let $H \in \mathcal{C}(\mathcal{X})$. Suppose $\alpha: \mathbb{S}^{1} \rightarrow \partial H$ is a $\left(\lambda^{\text {level }(H)}, L\right)$-quasisimilar meridian on $H$. Then, for each $m \geq 0$, there exists a $\left(\lambda^{\operatorname{level}(H)}, L^{\prime}\right)$-quasisimilarity

$$
\left.\varkappa_{\alpha}:\left(\mathbb{B}^{2+m} \times \mathbb{S}^{1},\{0\} \times \mathbb{S}^{1}\right) \rightarrow\left(N_{d_{\lambda, m}}\left(\pi_{G}(\partial H), \epsilon_{\lambda} \lambda^{\text {level }(H)}\right),\left(\pi_{G} \circ \alpha\right) \times\{0\}\right)\right),
$$

where $L^{\prime}>1$ is a constant depending only on $m, d_{\lambda}$ and $L$.

Given a defining sequence $\mathcal{X}=\left(X_{k}\right)$ and a union $Y$ of a nonempty subcollection of cubes-with-handles in $\mathcal{C}\left(X_{k}\right)$, we call

$$
\Sigma(Y, \mathcal{X})=\left\{\sigma \in \Sigma(Y):|\sigma| \subset X_{k} \backslash X_{k+1}\right\}
$$

the set of longitudes of $Y$ relative to $\mathcal{X}$. This subfamily $\Sigma\left(X_{k^{\prime}} \cap H, \mathcal{X}\right)$ of $\Sigma\left(X_{k^{\prime}} \cap H\right)$ can be used to determine the circulation $\operatorname{circ}\left(X_{k^{\prime}} \cap H, \alpha, H\right)$.

Lemma 9.5. Let $k \geq 0$ and $H \in \mathcal{C}\left(X_{k}\right)$, and let $\alpha$ be a meridian of $H$. Then

$$
\operatorname{circ}\left(X_{k^{\prime}} \cap H, \alpha, H\right)=\min _{\phi \in \mathcal{E}(H, \alpha)} \min _{\sigma \in \Sigma\left(X_{k^{\prime}} \cap H, \mathcal{X}\right)} \#\left(|\sigma| \cap \phi\left(\mathbb{B}^{2}\right)\right)
$$

for $k^{\prime}>k$.

Proof. Let $\sigma=\sigma_{1}+\cdots+\sigma_{\ell} \in \Sigma\left(X_{k^{\prime}} \cap H\right)$ and $\phi \in \mathcal{E}(H, \alpha)$ be chosen so that

$$
\#\left(|\sigma| \cap \phi\left(\mathbb{B}^{2}\right)\right)=\operatorname{circ}\left(X_{k^{\prime}} \cap H, \alpha, H\right) .
$$

We claim that there exists a homeomorphism $h$ of $X_{k^{\prime}} \cap H$, equal to the identity on $\partial\left(X_{k^{\prime}} \cap H\right)$, so that $h \circ \sigma=h \circ \sigma_{1}+\cdots+h \circ \sigma_{\ell} \in \Sigma\left(X_{k^{\prime}} \cap H, \mathcal{X}\right)$. 
For every component $H_{1}, \ldots, H_{d}$ of $X_{k^{\prime}} \cap H$ let $g_{i}$ be the genus of $H_{i}$ and let $\rho_{i}: \bigvee^{g_{i}} \mathbb{S}^{1} \rightarrow H_{i}$ be a core of $H_{i}$. Let $\mathcal{R}=\bigcup_{i} \rho_{i}\left(\bigvee^{g_{i}} \mathbb{S}^{1}\right)$. By considering an isotopy of $X_{k^{\prime}} \cap H$ if necessary, we may assume that $\mathcal{R} \cap|\sigma|=\emptyset$. Then there exists a regular neighborhood $X$ of $\mathcal{R}$ so that $\left(X_{k^{\prime}} \cap H\right) \backslash X$ is homeomorphic to $\partial\left(X_{k^{\prime}} \cap H\right) \times[0,1)$ and $|\sigma| \subset\left(X_{k^{\prime}} \cap H\right) \backslash X$. Then there exists a homeomorphism $h$ of $X_{k^{\prime}} \cap H$, isotopic to the identity, so that $h\left(\left(X_{k^{\prime}} \cap H\right) \backslash X\right) \cap X_{k^{\prime}+1}=\emptyset$ and $h$ is the identity on $\partial\left(X_{k^{\prime}} \cap H\right)$. Hence $h \circ \sigma \in \Sigma\left(X_{k^{\prime}} \cap H, \mathcal{X}\right)$.

We extend the homeomorphism $h$ by the identity on $H \backslash X_{k^{\prime}}$. Then $h \circ \phi \in$ $\mathcal{E}(H, \alpha)$ and

$$
\#\left(|h \circ \sigma| \cap h\left(\phi\left(\mathbb{B}^{2}\right)\right)\right)=\#\left(|\sigma| \cap \phi\left(\mathbb{B}^{2}\right)\right)=\operatorname{circ}\left(X_{k^{\prime}} \cap H, \alpha, H\right) .
$$

The claim follows from $\Sigma\left(X_{k^{\prime}} \cap H, \mathcal{X}\right) \subset \Sigma\left(X_{k^{\prime}} \cap H\right)$.

\subsection{Intersections in decomposition spaces}

When $\mathbb{R}^{3} / G$ is a manifold factor, the circulation of cubes-with-handles in $\mathbb{R}^{3}$ can be estimated from above by the intersection number of longitudes and interior essential components of maps in the decomposition space $\mathbb{R}^{3} / G$, instead of $\mathbb{R}^{3}$. The following proposition deals with this subtle, technical point.

In the following, $\Pi: \mathbb{R}^{3} / G \times \mathbb{R}^{m} \rightarrow \mathbb{R}^{3} / G$ is the projection map $(x, v) \mapsto x$.

Proposition 9.6. Let $\left(\mathbb{R}^{3} / G, \mathcal{X}\right)$ be a decomposition space, let $\alpha: \mathbb{S}^{1} \rightarrow \partial H$ be a meridian of $H \in \mathcal{C}(\mathcal{X})$, and let $\zeta: \mathbb{B}^{2} \rightarrow \pi_{G} H$ be a map satisfying $\zeta \mid \partial \mathbb{B}^{2}=\pi_{G} \circ \alpha$. Suppose that $\mathbb{R}^{3} / G \times \mathbb{R}^{m}$ is homeomorphic to $\mathbb{R}^{3+m}$ for some $m \geq 0$. Then

$$
\#\left(\pi_{G}(|\sigma|) \cap \zeta\left(\mathbb{B}^{2}\right)\right) \geq \operatorname{circ}\left(X_{k^{\prime}} \cap H, \alpha, H\right)
$$

for all $k^{\prime}>$ level $(H)$ and every longitude $\sigma \in \Sigma\left(X_{k^{\prime}} \cap H, \mathcal{X}\right)$.

The proof is based on the following approximation lemma.

Lemma 9.7. Under the hypotheses of the proposition, for every $k^{\prime}>k$ there exists a map $\phi: \mathbb{B}^{2} \rightarrow H$ so that $\pi_{G} \circ \phi|\Omega=\zeta| \Omega$, where $\Omega$ is the component of $\zeta^{-1}\left(\pi_{G} H \backslash \pi_{G}\left(X_{k^{\prime}}\right)\right)$ that contains $\partial \mathbb{B}^{2}$.

Proof. If $\zeta\left(\mathbb{B}^{2}\right) \cap \pi_{G}\left(X_{K}\right)=\emptyset$ for some $K>0$ then we may take $\phi=\pi_{G}^{-1} \circ \zeta$, since $\pi_{G} \mid \mathbb{R}^{3} \backslash X_{K}$ is a homeomorphism. The conclusion follows. Thus we may assume that $\zeta\left(\mathbb{B}^{2}\right) \cap \pi_{G}\left(X_{K}\right) \neq \emptyset$ for all $K>0$.

We fix a homeomorphism $f: \mathbb{R}^{3} / G \times \mathbb{R}^{m} \rightarrow \mathbb{R}^{3+m}$ and a number $R>0$ so that $f\left(\zeta\left(\mathbb{B}^{2}\right)\right) \subset B^{3+m}(R)$. Let $\mathcal{B}^{\prime}=B^{3+m}(R+1), \mathcal{B}=B^{3+m}(R+2)$, and

$$
\varepsilon=\frac{1}{4} \min \left\{1, \operatorname{dist}\left(f\left(\pi_{G}\left(\partial X_{k^{\prime}+1}\right) \times \mathbb{R}^{m}\right) \cap \mathcal{B}, f\left(\pi_{G}\left(X_{k^{\prime}+2}\right) \times \mathbb{R}^{m}\right) \cap \mathcal{B}\right)\right\} .
$$

Since $\zeta$ and $f \mid f^{-1} \mathcal{B}$ are uniformly continuous, we may fix $\delta>0$ so that $\mid f(\zeta(x))-$ $f(\zeta(y)) \mid<\varepsilon / 5$ for all $x, y \in \mathbb{B}^{2}$ satisfying $|x-y|<\delta$. 
We fix $K>k^{\prime}+2$ so that the diameters of components of $\zeta^{-1}\left(\pi_{G}\left(X_{K}\right)\right)$ are at most $\delta / 2$. Let $\Omega_{K}$ be the component of $\zeta^{-1}\left(\mathbb{R}^{3} / G \backslash \pi_{G}\left(X_{K}\right)\right)$ that contains $\partial \mathbb{B}^{2}$. Then $\Omega \subset \Omega_{K}$.

Since $\pi_{G}$ is a homeomorphism near the boundary of $X_{K}$, we may use the transversality and the PL-structure in $\mathbb{R}^{3}$ to modify $\zeta$ in a neighborhood of $\pi_{G}\left(\partial X_{K}\right)$ in $\pi_{G}\left(X_{k+2}\right)$ in such a way that the components of $\zeta^{-1}\left(\pi_{G}\left(\partial X_{K}\right)\right)$ are topological circles, that $|f(\zeta(x))-f(\zeta(y))|<\varepsilon / 4$ for all $|x-y|<\delta$, and that $f\left(\zeta\left(\mathbb{B}^{2}\right)\right) \subset$ $B^{3+m}(R+\varepsilon)$.

For each component $C$ of $\partial \Omega_{K}$, except for the outermost boundary $\partial \mathbb{B}^{2}$, we denote by $\omega$ the 2 -cell in $\mathbb{B}^{2}$ enclosed by $C$, thus $C=\partial \omega$, and define a map $\tilde{\phi}_{\omega}: \omega \rightarrow \mathbb{R}^{3+m}$ extending $f \circ \zeta \mid \partial \omega$ as follows.

Let $\tau: \omega \rightarrow \mathbb{B}^{2}$ be a homeomorphism and fix a point $y_{0} \in f(\zeta(\partial \omega))$. Define $\tilde{\phi}_{\omega}: \omega \rightarrow \mathbb{R}^{3+m}$ so that $\tilde{\phi}_{\omega}\left(\tau^{-1}(0)\right)=y_{0}$ and

$$
\tilde{\phi}_{\omega}(x)=(1-|\tau(x)|) y_{0}+|\tau(x)| f \circ \zeta \circ \tau^{-1}\left(\frac{\tau(x)}{|\tau(x)|}\right), \quad x \neq \tau^{-1}(0) .
$$

Then $\tilde{\phi}_{\omega}|\partial \omega=f \circ \zeta| \partial \omega$. Since $\operatorname{diam} \partial \omega<\delta$ we have $\operatorname{diam} f(\zeta(\partial \omega)) \leq \varepsilon / 4$, $\operatorname{diam}\left(\tilde{\phi}_{\omega}(\omega)\right)<\varepsilon$, and $\tilde{\phi}_{\omega}(\omega) \subset \mathcal{B}^{\prime} ;$ since $\zeta(\partial \omega) \subset \pi_{G}\left(\partial X_{K}\right)$, we have $\left.\tilde{\phi}_{\omega}(\partial \omega)\right) \subset$ $f\left(\pi_{G}\left(X_{k^{\prime}+2}\right) \times \mathbb{R}^{m}\right) \cap \mathcal{B}^{\prime}$. Therefore

$$
\begin{aligned}
\operatorname{dist}\left(\tilde{\phi}_{\omega}(\omega), f\left(\pi_{G}\left(\partial X_{k^{\prime}+1}\right) \times \mathbb{R}^{m}\right)\right) \\
\quad \geq \operatorname{dist}\left(\tilde{\phi}_{\omega}(\partial \omega), f\left(\pi_{G}\left(\partial X_{k^{\prime}+1}\right) \times \mathbb{R}^{m}\right)\right)-\operatorname{diam}\left(\tilde{\phi}_{\omega}(\omega)\right) \\
\quad \geq \min \left\{1, \operatorname{dist}\left(\tilde{\phi}_{\omega}(\partial \omega), f\left(\pi_{G}\left(\partial X_{k^{\prime}+1}\right) \times \mathbb{R}^{m}\right) \cap \mathcal{B}\right)\right\}-\operatorname{diam}\left(\tilde{\phi}_{\omega}(\omega)\right)>3 \varepsilon
\end{aligned}
$$

Thus $\tilde{\phi}_{\omega}(\omega) \subset f\left(\pi_{G}\left(X_{k^{\prime}+1}\right) \times \mathbb{R}^{m}\right)$.

We define a map $\phi: \mathbb{B}^{2} \rightarrow \mathbb{R}^{3}$ by $\phi\left|\Omega_{K}=\pi_{G}^{-1} \circ \zeta\right| \Omega_{K}$ and $\phi \mid \omega=\Pi \circ f^{-1} \circ \tilde{\phi}_{\omega}$ on every 2-cell $\omega$ bounded by a component of $\partial \Omega_{K} \backslash \partial \mathbb{B}^{2}$. Since $\pi_{G} \mid \mathbb{R}^{3} \backslash X_{K}$ is a homeomorphism and $\tilde{\phi}_{\omega}|\partial \omega=f \circ \zeta| \partial \omega$, the map is well-defined and continuous.

Since $\phi\left(\mathbb{B}^{2}\right)$ is connected, $\phi\left(\mathbb{B}^{2}\right) \subset H$, and since $\Omega \subset \Omega_{K}, \phi\left|\Omega=\pi_{G}^{-1} \circ \zeta\right| \Omega$. The claim follows.

Proof of Proposition 9.6. The map $\phi: \mathbb{B}^{2} \rightarrow H$ constructed in Lemma 9.7 belongs to $\mathcal{E}(H ; \alpha)$ and satisfies $\pi_{G} \circ \phi|\Omega=\zeta| \Omega$. Then, by Lemma 9.5 ,

$$
\#\left(\zeta\left(\mathbb{B}^{2}\right) \cap \pi_{G}(|\sigma|)\right) \geq \#\left(\phi\left(\mathbb{B}^{2}\right) \cap|\sigma|\right) \geq \operatorname{circ}\left(X_{k^{\prime}} \cap H, \alpha, H\right)
$$

for every $\sigma \in \Sigma\left(X_{k^{\prime}} \cap H, \mathcal{X}\right)$. The claim follows.

\subsection{Virtually interior essential components}

Let $\Omega$ be a 2-manifold with boundary, let $M$ be an $n$-manifold with boundary, and let $\phi:(\Omega, \partial \Omega) \rightarrow(M, \partial M)$ be a map. Following Daverman ([6], pp. 73-74), we say that $\phi$ is interior inessential if there exists a map $\phi^{\prime}: \Omega \rightarrow \partial M$ so that $\phi^{\prime}|\partial \Omega=\phi| \partial \Omega$; if no such map exists, we say that $\phi$ is interior essential. 
If $\phi:(\Omega, \partial \Omega) \rightarrow(M, \partial M)$ is interior essential and $\Omega$ is a submanifold of a 2-cell $D$ so that $\partial D \subset \partial \Omega$, we say that $\phi$ is virtually interior essential if there exists a map $\Phi: D \rightarrow M$ so that $\Phi \mid \Omega=\phi$ and $\Phi(D \backslash \Omega) \subset \partial M$.

Let $\mathcal{X}$ be a defining sequence for a decomposition space. Given $H \in \mathcal{C}\left(X_{k}\right)$, a meridian $\alpha$ of $H$, and $k^{\prime}>k$, denote by $\mathcal{E}\left(H, \alpha ; X_{k^{\prime}}\right)$ the collection of maps $\phi:\left(\mathbb{B}^{2}, \partial \mathbb{B}^{2}\right) \rightarrow(H, \alpha)$ such that $\phi\left(\mathbb{B}^{2}\right)$ is transverse to $\partial X_{k^{\prime}}$. Given $\phi \in \mathcal{E}\left(H, \alpha ; X_{k^{\prime}}\right)$, we say that a component $\omega$ of $\phi^{-1} X_{k^{\prime}}$ is virtually interior essential with respect to $X_{k^{\prime}}$ if $\phi \mid \omega:(\omega, \partial \omega) \rightarrow\left(X_{k^{\prime}}, \partial X_{k^{\prime}}\right)$ is virtually interior essential. We denote by $\Gamma\left(\phi, X_{k^{\prime}}\right)$ the set of virtually interior essential components of $\phi^{-1} X_{k^{\prime}}$.

Remark 9.8. The circulation $\operatorname{circ}\left(X_{k^{\prime}} \cap H, \alpha, H\right)$ is closely related to the minimal number of essential components among all maps in $\mathcal{E}\left(H, \alpha ; X_{k^{\prime}}\right)$. In fact,

$$
\operatorname{circ}\left(X_{k^{\prime}} \cap H, \alpha, H\right) \geq \min _{\phi \in \mathcal{E}\left(H, \alpha ; X_{k^{\prime}}\right)} \# \Gamma\left(\phi, X_{k^{\prime}}\right) .
$$

Indeed, given $\phi \in \mathcal{E}\left(H, \alpha ; X_{k^{\prime}}\right)$ and $\sigma \in \Sigma\left(X_{k^{\prime}} \cap H\right)$, it follows from the definition of longitudes that $|\sigma| \cap \phi(\omega) \neq \emptyset$ for every $\omega \in \Gamma\left(\phi, X_{k^{\prime}}\right)$. Thus $\#\left(|\sigma| \cap \phi\left(\mathbb{B}^{2}\right)\right) \geq \# \Gamma\left(\phi, X_{k^{\prime}}\right)$.

\section{Circulation and a modulus estimate for walls}

Suppose that $\left(\mathbb{R}^{3} / G, \mathcal{X},(\mathcal{C}, \mathcal{A}, \mathcal{W}), \theta, d_{\lambda}\right)$ is a Semmes space. Let $Y$ be the union of a nonempty subcollection of cubes-with-handles in $\mathcal{C}\left(X_{k}\right)$, and let $m \geq 0$ and $a>0$. We call carriers of $(1+m)$-chains in the collection

$$
\Sigma^{m}(Y, \mathcal{X}, a)=\left\{|\sigma| \times[-a, a]^{m}: \sigma \in \Sigma(Y, \mathcal{X})\right\}
$$

$m$-walls over $Y$ of height a relative to $\mathcal{X}$. Note that these walls do not meet $X_{\infty} \times \mathbb{R}^{m}$ and that $\pi_{G} \mid \mathbb{R}^{3} \backslash X_{\infty}$ is a homeomorphism. We denote by

$$
\hat{\Sigma}^{m}(Y, \mathcal{X}, a)=\left(\pi_{G} \times \mathrm{id}\right)\left(\Sigma^{m}(Y, \mathcal{X}, a)\right)
$$

the corresponding collection of $m$-walls in the decomposition space $\mathbb{R}^{3} / G \times \mathbb{R}^{m}$.

The main result of this section is an upper bound for the conformal modulus of an $m$-wall family in terms of circulation. This, together with a lower bound in terms of growth, yields a necessary condition for the existence of a quasisymmetric parametrization. Our result extends the second part of Proposition 4.5 in [12].

Theorem 10.1. Let $\left(\mathbb{R}^{3} / G, \mathcal{X},(\mathcal{C}, \mathcal{A}, \mathcal{W}), \theta, d_{\lambda}\right)$ be a Semmes space, let $\alpha: \mathbb{S}^{1} \rightarrow$ $\partial H$ be an $\left(\lambda^{k}, L\right)$-quasisimilar meridian of $H \in \mathcal{C}\left(X_{k}\right)$, and let $m \geq 0$. Suppose that $f:\left(\mathbb{R}^{3} / G \times \mathbb{R}^{m}, d_{\lambda, m}\right) \rightarrow \mathbb{R}^{3+m}$ is $\eta$-quasisymmetric. Then there exist $A=$ $A\left(\eta, d_{\lambda}, m, L\right)>0$ and $C=C\left(\eta, d_{\lambda}, m, L\right)$ so that

$$
\operatorname{Mod}_{\frac{3+m}{1+m}}\left(f\left(\hat{\Sigma}^{m}\left(X_{k^{\prime}} \cap H, \mathcal{X}, A \lambda^{k}\right)\right)\right) \leq C\left(\frac{1}{\operatorname{circ}\left(X_{k^{\prime}} \cap H, \alpha, H\right)}\right)^{(3+m) /(1+m)}
$$

for all $k^{\prime}>k+1$. 
We begin with an intersection lemma which contains the gist of the proof; the number $\epsilon_{\lambda}$ in the statement is the constant defined in Remark 7.7.

Lemma 10.2. Suppose $g:\left(\mathbb{R}^{3} / G \times \mathbb{R}^{m}, d_{\lambda, m}\right) \rightarrow \mathbb{R}^{3+m}$ is $\eta$-quasisymmetric. Let $\alpha: \mathbb{S}^{1} \rightarrow \partial H$ be a meridian of $H \in \mathcal{C}(\mathcal{X})$ and let $\beta: \mathbb{S}^{1} \rightarrow \mathbb{R}^{3} / G \times \mathbb{R}^{m}$ be a map homotopic to $\pi_{G} \circ \alpha$ in $N_{d_{\lambda, m}}\left(\pi_{G}(\partial H), \epsilon_{\lambda} \lambda^{\operatorname{level}(H)} / 3\right)$ with the property that $g\left(\beta\left(\mathbb{S}^{1}\right)\right)=\partial \mathbb{B}^{2} \times\{0\} \subset \mathbb{R}^{2} \times \mathbb{R}^{1+m}$. Then there exist $\delta=\delta\left(\eta, d_{\lambda, m}\right)>0$ and $A=A\left(\eta_{g-1}\right) \geq 1$ so that

$$
\#\left(g(w) \cap\left(\mathbb{B}^{2}+j\right)\right) \geq \operatorname{circ}\left(X_{k^{\prime}} \cap H, \alpha, H\right)
$$

for every $k^{\prime}>\operatorname{level}(H), m$-wall $w \in \hat{\Sigma}^{m}\left(X_{k^{\prime}} \cap H, \mathcal{X}, A \lambda^{\operatorname{level}(H)}\right)$, and $j \in\{0\} \times$ $B^{1+m}(\delta) \subset \mathbb{R}^{2} \times \mathbb{R}^{1+m}$.
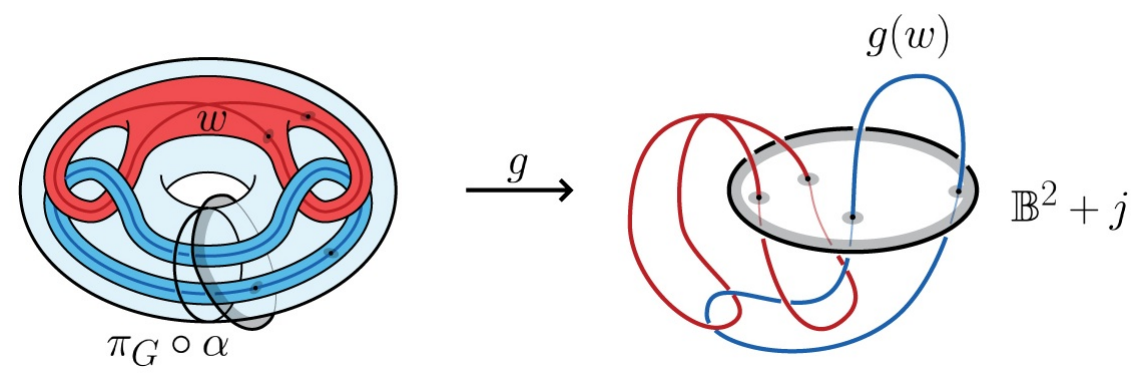

Figure 8. An example of (10.1), where $m=0, k=0$, and $k^{\prime}=1$.

Proof. Let $k=\operatorname{level}(H)$. We show first that $\operatorname{dist}\left(\partial \mathbb{B}^{2}, g\left(\pi_{G}\left(X_{k+1}\right) \times \mathbb{R}^{m}\right)\right)$ is bounded from below by a positive constant depending only on $\eta$ and $\lambda$.

Since $\beta\left(\mathbb{S}^{1}\right) \subset N_{d_{\lambda, m}}\left(\pi_{G}(\partial H), \epsilon_{\lambda} \lambda^{k} / 3\right) \subset \pi_{G}\left(\mathbb{R}^{3} \backslash X_{k+1}\right) \times \mathbb{R}^{m}$ and $g\left(\beta\left(\mathbb{S}^{1}\right)\right)=$ $\partial \mathbb{B}^{2}$, we may fix $x \in \beta\left(\mathbb{S}^{1}\right)$ and $z \in \pi_{G}\left(X_{k+1}\right) \times \mathbb{R}^{m}$ so that

$$
|g(x)-g(z)|=\operatorname{dist}\left(\partial \mathbb{B}^{2}, g\left(\pi_{G}\left(X_{k+1}\right) \times \mathbb{R}^{m}\right)\right) .
$$

We also fix $y \in \beta\left(\mathbb{S}^{1}\right)$ so that $d_{\lambda, m}(x, y)=d_{\lambda}(x, y)=\max _{y^{\prime} \in \beta\left(\mathbb{S}^{1}\right)} d_{\lambda}\left(x, y^{\prime}\right)$. Since $x, y \in N_{d_{\lambda, m}}\left(\pi_{G}(\partial H), \epsilon_{\lambda} \lambda^{k}\right)$ and projection $\Pi(z) \in \pi_{G}\left(X_{k+1}\right)$, we have, by quasisymmetry and Remark 7.5,

$$
\begin{aligned}
|g(x)-g(y)| & \leq \eta\left(\frac{d_{\lambda, m}(x, y)}{d_{\lambda, m}(x, z)}\right)|g(x)-g(z)| \\
& \leq \eta\left(\frac{\operatorname{diam}_{d_{\lambda, m}}\left(N_{d_{\lambda, m}}\left(\pi_{G}(\partial H), \epsilon_{\lambda} \lambda^{k}\right)\right)}{\operatorname{dist}_{d_{\lambda, m}}\left(N_{d_{\lambda, m}}\left(\pi_{G}(\partial H), \pi_{G}\left(X_{k+1}\right)\right)\right.}\right)|g(x)-g(z)| \\
& \leq \eta\left(C\left(d_{\lambda, m}\right)\right)|g(x)-g(z)| .
\end{aligned}
$$

Choose $x^{\prime} \in \beta\left(\mathbb{S}^{1}\right)$ so that $g\left(x^{\prime}\right)$ and $g(x)$ are antipodal on $\partial \mathbb{B}^{2}$. Then,

$$
\left|g(x)-g\left(x^{\prime}\right)\right| \leq \eta\left(\frac{d_{\lambda, m}\left(x, x^{\prime}\right)}{d_{\lambda, m}(x, y)}\right)|g(x)-g(y)| \leq \eta(1)|g(x)-g(y)| .
$$


Thus

$$
\operatorname{dist}\left(\partial \mathbb{B}^{2}, g\left(\pi_{G}\left(X_{k+1}\right) \times \mathbb{R}^{m}\right)\right) \geq \frac{2}{\eta(1) \eta\left(C\left(d_{\lambda, m}\right)\right)}
$$

Since $g$ is $\eta$-quasisymmetric, we may fix $\delta \in\left(0,\left(\eta(1) \eta\left(C\left(d_{\lambda, m}\right)\right)\right)^{-1}\right)$, independent of $k$, so that

$$
g^{-1}\left(\partial \mathbb{B}^{2} \times B^{1+m}(\delta)\right) \subset N_{d_{\lambda, m}}\left(\pi_{G}(\partial H), \epsilon_{\lambda} \lambda^{k}\right) .
$$

We prove now (10.1). Let $k^{\prime}>k$ and $j \in\{0\} \times B^{1+m}(\delta)$; define $\phi_{j}:\left(\mathbb{B}^{2}, \partial \mathbb{B}^{2}\right) \rightarrow$ $\mathbb{R}^{3} / G \times \mathbb{R}^{m}$ to be the map $\phi_{j}(x)=g^{-1}(x+j)$.

By the definition of $\epsilon_{\lambda}, N_{d_{\lambda}}\left(\pi_{G}(\partial H), \epsilon_{\lambda} \lambda^{k}\right) \subset \mathbb{R}^{3} / G$ is contained in a regular neighborhood of $\pi_{G}(\partial H)$. In view of (10.2) and (10.3), the projection $\Pi \circ \phi_{j} \mid \partial \mathbb{B}^{2}$ is homotopic to $\pi_{G} \circ \alpha$ in $N_{d_{\lambda}}\left(\pi_{G}(\partial H), \epsilon_{\lambda} \lambda^{k}\right) \subset \mathbb{R}^{3} / G$ and there exists a map $\zeta:\left(\mathbb{B}^{2}, \partial \mathbb{B}^{2}\right) \rightarrow\left(\pi_{G} H, \pi_{G} \circ \alpha\right)$ so that $\zeta\left|\Omega=\Pi \circ \phi_{j}\right| \Omega$, where $\Omega=\left(\Pi \circ \phi_{j}\right)^{-1}\left(\pi_{G}\left(X_{k^{\prime}}\right)\right)$.

Note, from Proposition 9.6, that

$$
\#\left(\pi_{G}(|\sigma|) \cap \zeta\left(\mathbb{B}^{2}\right)\right) \geq \operatorname{circ}\left(X_{k^{\prime}} \cap H, \alpha, H\right)
$$

for every $\sigma \in \Sigma\left(X_{k^{\prime}} \cap H, \mathcal{X}\right)$. Thus

$$
\begin{aligned}
\#\left(g\left(\pi_{G}(|\sigma|) \times \mathbb{R}^{m}\right) \cap\left(\mathbb{B}^{2}+j\right)\right) & =\#\left(\left(\pi_{G}(|\sigma|) \times \mathbb{R}^{m}\right) \cap g^{-1}\left(\mathbb{B}^{2}+j\right)\right) \\
& =\#\left(\left(\pi_{G}(|\sigma|) \times \mathbb{R}^{m}\right) \cap \phi_{j}\left(\mathbb{B}^{2}\right)\right) \\
& \geq \#\left(\pi_{G}(|\sigma|) \cap \Pi \phi_{j}\left(\mathbb{B}^{2}\right)\right) \\
& =\#\left(\pi_{G}(|\sigma|) \cap \zeta\left(\mathbb{B}^{2}\right)\right) \\
& \geq \operatorname{circ}\left(X_{k^{\prime}} \cap H, \alpha, H\right)
\end{aligned}
$$

for all $\sigma \in \Sigma\left(X_{k^{\prime}} \cap H, \mathcal{X}\right)$. This concludes the proof in the case $m=0$.

Suppose now $m \geq 1$. It suffices to find $A=A(\eta) \geq 1$ so that

$$
\phi_{j}\left(\mathbb{B}^{2}\right) \subset \mathbb{R}^{3} / G \times\left[-A \lambda^{k}, A \lambda^{k}\right]^{m} .
$$

Let $x \in \partial \mathbb{B}^{2}$ and $y \in \mathbb{B}^{2}$. By quasisymmetry of $g^{-1}$, we have

$$
\begin{aligned}
\left|\phi_{j}(y)-\phi_{j}(x)\right| & =\left|g^{-1}(y+j)-g^{-1}(x+j)\right| \\
& \leq \eta_{g^{-1}}\left(\frac{|y-x|}{|(-x)-(x+j)|}\right)\left|g^{-1}(-x)-g^{-1}(x+j)\right| \\
& \leq \eta_{g^{-1}}\left(\frac{|y-x|}{2-|j|}\right) \eta_{g^{-1}}\left(\frac{2+|j|}{2}\right)\left|g^{-1}(-x)-g^{-1}(x)\right| \\
& \leq \eta_{g^{-1}}(1) \eta_{g^{-1}}(2) \operatorname{diam}_{d_{\lambda, m}} g^{-1}\left(\partial \mathbb{B}^{2}\right) .
\end{aligned}
$$

Since

$$
g^{-1}\left(\partial \mathbb{B}^{2}\right)=|\beta| \subset N_{d_{\lambda, m}}\left(\pi_{G}(\partial H), \epsilon_{\lambda} \lambda^{k}\right),
$$

(10.4) holds with $A=C(\lambda) \eta_{g^{-1}}(1) \eta_{g^{-1}}(2)$. The claim now follows. 
The proof of Theorem 10.1 is based on Lemma 10.2 and unknotting properties of quasisymmetric tubes; see Propositions 11.1 and 11.3.

Proof of Theorem 10.1. We first consider the case $m \geq 1$. Let $\alpha: \mathbb{S}^{1} \rightarrow \partial H$ be the $\left(\lambda^{k}, L\right)$-quasisimilar meridian in the statement of the theorem and let $k^{\prime}>k+1$. We assume, as we may, that $\operatorname{circ}\left(X_{k^{\prime}} \cap H, \alpha, H\right)>0$. So

$$
\#\left(|\sigma| \cap \phi\left(\mathbb{B}^{2}\right)\right) \geq \operatorname{circ}\left(X_{k^{\prime}} \cap H, \alpha, H\right)
$$

for all $\sigma \in \Sigma\left(X_{k^{\prime}} \cap H, \mathcal{X}\right)$ and all maps $\phi:\left(\mathbb{B}^{2}, \partial \mathbb{B}^{2}\right) \rightarrow(H, \alpha)$.

By Lemma 9.4, there exists an $\left(\lambda^{k}, L^{\prime}\right)$-quasisimilar, thus $\eta^{\prime}$-quasisymmetric, embedding $\varkappa: \mathbb{B}^{2+m} \times \mathbb{S}^{1} \rightarrow N_{d_{\lambda, m}}\left(\pi_{G}(\partial H), \epsilon_{\lambda} \lambda^{k} / 3\right)$ so that $\varkappa(0, x)=\left(\pi_{G} \circ \alpha(x), 0\right)$ for $x \in \mathbb{S}^{1}$, where the constant $L^{\prime}$ and the homeomorphism $\eta^{\prime}:[0, \infty) \rightarrow[0, \infty)$ depend only on $d_{\lambda}, m$ and $L$. Recall that $N_{d_{\lambda, m}}\left(\pi_{G}(\partial H), \epsilon_{\lambda} \lambda^{k}\right) \subset \pi_{G}\left(\mathbb{R}^{3} \backslash X_{k+1}\right) \times \mathbb{R}^{m}$.

Set $T=f \circ \varkappa\left(\mathbb{B}^{2+m} \times \mathbb{S}^{1}\right) \subset \mathbb{R}^{3+m}$ and $h=f \circ \varkappa$. By Proposition 11.1, there exist an $\eta^{\prime \prime}$-quasisymmetric map $\chi: \mathbb{R}^{3+m} \rightarrow \mathbb{R}^{3+m}, \eta^{\prime \prime}=\eta^{\prime \prime}\left(m, \eta, \eta^{\prime}\right)$, and a constant $\delta_{0}=\delta_{0}\left(m, \eta, \eta^{\prime}\right)>0$ so that $\chi(T)$ contains the tubular neighborhood $N^{3+m}\left(\partial \mathbb{B}^{2}, 2 \delta_{0}\right)$ of $\partial \mathbb{B}^{2}$ in $\mathbb{R}^{3+m}$ and that $\chi \circ f \circ \pi_{G} \circ \alpha: \mathbb{S}^{1} \rightarrow \mathbb{R}^{3+m}$ is homotopic in $\chi(T)$ to the identity map id: $\partial \mathbb{B}^{2} \rightarrow \mathbb{R}^{2} \times \mathbb{R}^{1+m}$. Set $\beta=$ $f^{-1} \circ \chi^{-1} \circ \operatorname{id}_{\mathbb{R}^{3+m}} \mid \mathbb{S}^{1}: \mathbb{S}^{1} \rightarrow \mathbb{R}^{3} / G \times \mathbb{R}^{m}$. Thus we have

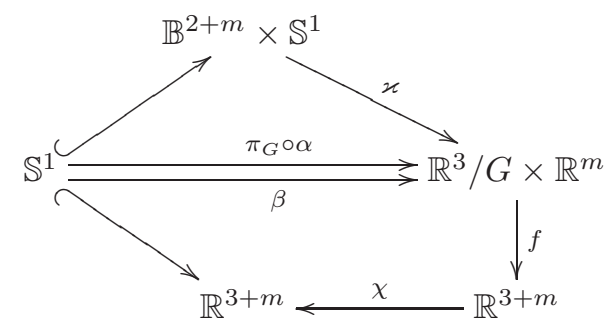

where both diagrams commute and the maps $\pi_{G} \circ \alpha$ and $\beta$ are homotopic in $\varkappa\left(\mathbb{B}^{2+m} \times \mathbb{S}^{1}\right)$.

Note that $\chi \circ f: \mathbb{R}^{3} / G \times \mathbb{R}^{m} \rightarrow \mathbb{R}^{3+m}$ is $\eta^{\prime \prime \prime}$-quasisymmetric for some $\eta^{\prime \prime \prime}=$ $\eta^{\prime \prime \prime}\left(\eta, \eta^{\prime \prime}\right)$.

Since $\beta$ is homotopic to $\pi_{G} \circ \alpha$ in $\varkappa\left(\mathbb{B}^{2+m} \times \mathbb{S}^{1}\right) \subset N_{d_{\lambda, m}}\left(\pi_{G}(\partial H), \epsilon_{\lambda} \lambda^{k} / 3\right)$ and $\operatorname{dist}\left(\partial \mathbb{B}^{2}, \chi \circ f\left(\pi_{G}\left(X_{k+1}\right) \times \mathbb{R}^{m}\right)\right)>2 \delta_{0}$, we can find, by applying Lemma 10.2 to $g=\chi \circ f$ and setting $\delta=\delta_{0}$, a constant $A=A\left(\eta^{\prime \prime \prime}, m\right)$ so that

$$
\#\left(\chi(f(w)) \cap\left(\mathbb{B}^{2}+j\right)\right) \geq \operatorname{circ}\left(X_{k^{\prime}} \cap H, \alpha, H\right),
$$

for every $w \in \hat{\Sigma}^{m}\left(X_{k^{\prime}}, \mathcal{X}, A \lambda^{k}\right)$ and $j \in\{0\} \times B^{1+m}\left(\delta_{0}\right)$.

Using (10.5) we estimate the conformal modulus of the $m$-wall family $\chi \circ$ $f\left(\hat{\Sigma}^{m}\left(X_{k^{\prime}}, \mathcal{X}, A \lambda^{k}\right)\right)$ in $\mathbb{R}^{3+m}$. Set $J=\{0\} \times B^{1+m}\left(\delta_{0}\right)$. By the co-area formula, we have, for $w \in \hat{\Sigma}^{m}\left(X_{k^{\prime}}, \mathcal{X}, A \lambda^{k}\right)$, that

$$
\begin{aligned}
\mathcal{H}^{1+m} & (\chi(f(w))) \geq \mathcal{H}^{1+m}\left(\chi(f(w)) \cap\left(\mathbb{B}^{2} \times B^{1+m}\left(\delta_{0}\right)\right)\right) \\
& \geq \int_{J} \#\left(\chi(f(w)) \cap\left(\mathbb{B}^{2}+j\right)\right) \mathrm{d} \mathcal{H}^{1+m}(j) \geq \operatorname{circ}\left(X_{k^{\prime}} \cap H, \alpha, H\right) \mathcal{H}^{1+m}(J) .
\end{aligned}
$$


Thus

$$
\rho=\frac{1}{\operatorname{circ}\left(X_{k^{\prime}} \cap H, \alpha, H\right) \mathcal{H}^{1+m}(J)} \chi_{\mathbb{B}^{2} \times J},
$$

is an admissible function for the family $\chi\left(f\left(\hat{\Sigma}^{m}\left(X_{k^{\prime}}, \mathcal{X}, A \lambda^{k}\right)\right)\right)$, and

$$
\begin{aligned}
\operatorname{Mod}_{\frac{3+m}{1+m}} & \left(\chi\left(f \hat{\Sigma}^{m}\left(X_{k^{\prime}}, \mathcal{X}, A \lambda^{k}\right)\right) \leq \int_{\mathbb{R}^{3+m}} \rho^{(3+m) /(1+m)} \mathrm{d} \mathcal{H}^{3+m}\right. \\
& =\frac{\mathcal{H}^{2}\left(\mathbb{B}^{2}\right) \mathcal{H}^{m+1}(J)}{\mathcal{H}^{m+1}(J)^{(3+m) /(1+m)}}\left(\frac{1}{\operatorname{circ}\left(X_{k^{\prime}} \cap H, \alpha, H\right)}\right)^{(3+m) /(1+m)} \\
& \leq C\left(\delta_{0}, m\right)\left(\frac{1}{\operatorname{circ}\left(X_{k^{\prime}} \cap H, \alpha, H\right)}\right)^{(3+m) /(1+m)}
\end{aligned}
$$

This concludes the proof for $m \geq 1$.

In the case $m=0$, we apply Proposition 11.3 to the mapping $h=f \circ \varkappa$ and the 3-manifold $M=f\left(\pi_{G}(H)\right)$. Otherwise the proof is the same.

\section{Quasisymmetric tubes}

In Proposition 11.1 we quantify Zeeman's unknotting theorem to provide a quasisymmetric unknotting of quasisymmetric tubes in $\mathbb{R}^{n}, n \geq 4$. In Proposition 11.3 we apply Dehn's Lemma to treat the unknotting in $\mathbb{R}^{3}$.

Proposition 11.1. Let $m \geq 1$, let $h: \mathbb{B}^{2+m} \times \mathbb{S}^{1} \rightarrow \mathbb{R}^{3+m}$ be an $\eta$-quasisymmetric embedding, and let $T=h\left(\mathbb{B}^{2+m} \times \mathbb{S}^{1}\right)$. Then there exist an $\eta^{\prime}$-quasisymmetric homeomorphism $\chi: \mathbb{R}^{3+m} \rightarrow \mathbb{R}^{3+m}, \eta^{\prime}=\eta^{\prime}(m, \eta)$, and a constant $\delta_{0}=\delta_{0}(m, \eta)>$ 0 so that

(1) $\chi(T)$ contains the tube $N^{3+m}\left(\partial \mathbb{B}^{2}, \delta_{0}\right)$ in $\mathbb{R}^{3+m}$,

$(1)^{\prime}$ in particular, $\partial \mathbb{B}^{2}+j \subset \chi(T)$ for $j \in\{0\} \times B^{1+m}\left(\delta_{0}\right) \subset \mathbb{R}^{2} \times \mathbb{R}^{1+m}$, and

(2) $\chi \circ h \mid\left(\{0\} \times \mathbb{S}^{1}\right)$ is homotopic to the identity map id $\mid \partial \mathbb{B}^{2}$ in $\chi(T)$.

Here $\mathbb{B}^{2+m} \times \mathbb{S}^{1}$ has the natural Euclidean metric inherited from $\mathbb{R}^{2+m} \times \mathbb{R}^{2}=\mathbb{R}^{3+m}$.

We first state a bilipschitz version of Zeeman's theorem on unknotting a PL 1 -sphere in $\mathbb{S}^{q}$ for $q \geq 4$. Since the claim follows from Theorem 5.6 and Corollary 5.9 in [16] almost directly, we omit the details.

For the statement, let $\ell \in \mathbb{Z}_{+}$and $m \geq 1$. Given $w_{1}, \ldots, w_{n} \in(1 / \ell) \mathbb{Z}^{3+m}$, we set $w=\left(w_{1}, \ldots, w_{n}\right)$, and let $\gamma_{w}$ be the piecewise linear curve $\left[w_{0}, w_{1}\right] \cup\left[w_{1}, w_{2}\right] \cup$ $\cdots \cup\left[w_{n-1}, w_{0}\right]$ in $\mathbb{R}^{3+m}$. Given $R>0$, we also denote by $\mathcal{J}(R, \ell, m ; n)$ the collection of Jordan curves in $\left\{\gamma_{w} \subset B^{3+m}(R): w \in\left((1 / \ell) \mathbb{Z}^{3+m}\right)^{n}\right\}$.

Lemma 11.2. Let $R \geq 1, \ell \in \mathbb{Z}_{+}, m \geq 1$, and $n \geq 3$. Then there exists $L_{0}=L_{0}(R, \ell, m, n)$ so that given $\gamma \in \mathcal{J}(R, \ell, m ; n)$ there exists an $L_{0}$-bilipschitz map $\chi: \mathbb{R}^{3+m} \rightarrow \mathbb{R}^{3+m}$ satisfying $\chi(\gamma)=\partial B^{2}(\operatorname{diam} \gamma) \times\{0\} \subset \mathbb{R}^{2} \times \mathbb{R}^{1+m}$. 
Proof of Proposition 11.1. Set $\mathbb{S}^{1}=\{0\} \times \mathbb{S}^{1}$. Then, by quasisymmetry,

$$
\operatorname{diam} h\left(\mathbb{S}^{1}\right) \leq \eta(5) \operatorname{dist}\left(h\left(\mathbb{S}^{1}\right), \partial T\right) .
$$

Indeed, set $\kappa=\operatorname{dist}\left(h\left(\mathbb{S}^{1}\right), \partial T\right)$ and choose $x \in \mathbb{S}^{1}$ and $y \in \partial\left(\mathbb{B}^{2+m} \times \mathbb{S}^{1}\right)$ so that $|h(x)-h(y)|=\kappa$. Then

$$
\left|h\left(x^{\prime}\right)-h(x)\right| \leq \eta\left(\frac{\left|x^{\prime}-x\right|}{|y-x|}\right)|h(y)-h(x)| \leq \eta(5) \kappa
$$

for all $x^{\prime} \in \mathbb{S}^{1}$.

We fix an orientation of $h\left(\mathbb{S}^{1}\right)$ and choose points $z_{0}, z_{1}, \ldots, z_{n}=z_{0}$ on $h\left(\mathbb{S}^{1}\right)$ as follows. Let $z_{0}$ be any point on $h\left(\mathbb{S}^{1}\right)$. After $z_{i}$ has been chosen, let $z_{i+1}$ be the last point $z$ on the subarc of $h\left(\mathbb{S}^{1}\right)$ starting at $z_{i}$ and ending at $z_{0}$ according to the orientation, so that $\left|z-z_{i}\right|=\kappa / 100$ if such a point exists; otherwise, we have $\left|z_{0}-z_{i}\right|<\kappa / 100$ and in this case we remove the already defined value of $z_{i}$ and set $n=i$ and $z_{n}=z_{0}$. We show next that $n \leq n_{0}$ for some $n_{0}=n_{0}(\eta)>0$.

Let $s_{i}=h^{-1}\left(z_{i}\right) \in \mathbb{S}^{1}$ for $0 \leq i \leq n-1$. Then there exists an $i$ so that $\left|s_{i}-s_{i+1}\right| \leq 2 \pi / n$; for this particular $i$,

$$
\kappa / 100 \leq\left|z_{i}-z_{i+1}\right| \leq \eta\left(\frac{\left|s_{i}-s_{i+1}\right|}{\left|\left(-s_{i}\right)-s_{i}\right|}\right)\left|h\left(-s_{i}\right)-h\left(s_{i}\right)\right| \leq \eta(1 / n) \eta(5) \kappa .
$$

Hence $\left|s_{i}-s_{i+1}\right| \geq C_{0}$, where $C_{0}$ depends on $\eta$, and $n \leq 2 \pi / C_{0}$.

We next fix points $w_{i} \in\left(\kappa /\left(1000 \sqrt{m} n_{0}\right)\right) \mathbb{Z}^{3+m}$ so that $\left|w_{i}-z_{i}\right|<\kappa / 500$ and let $\gamma$ be the polygonal path $\left[w_{0}, w_{1}\right] \cup\left[w_{1}, w_{2}\right] \cup \cdots \cup\left[w_{n-1}, w_{0}\right]$.

By replacing the points $w_{i}$ with points in

$$
\left(\kappa /\left(1000 \sqrt{3+m} n_{0}\right)\right) \mathbb{Z}^{3+m} \cap B^{3+m}\left(z_{i}, \kappa / 500\right),
$$

we may assume that $\gamma$ is a Jordan curve. Indeed, if $\gamma$ is not a Jordan curve, then there exist indices $i$ and $j, i>j$, so that $\left(w_{i}, w_{i+1}\right) \cap\left(w_{j}, w_{j+1}\right) \neq \emptyset$. Since $B^{3+m}\left(z_{i}, \kappa / 500\right)$ contains more than $n_{0}^{3+m}$ points in $\left(\kappa /\left(1000 \sqrt{3+m} n_{0}\right)\right) \mathbb{Z}^{3+m}$ and there are at most $n_{0}\left(n_{0}-1\right) / 2$ directions between the points $w_{1}, \ldots, w_{n}$, there exists $w^{\prime} \in B^{3+m}\left(z_{i}, \kappa / 500\right)$ so that $\left(w_{i}, w^{\prime}\right) \cap\left(w_{k}, w_{k+1}\right)=\emptyset$ for all $k<i$. We remove all the intersections inductively on $i$.

Since $\max _{w \in\left[w_{i}, w_{i+1}\right]} \operatorname{dist}\left(w, z_{i}\right) \leq \kappa / 40$, we have $\max _{w \in \gamma} \operatorname{dist}\left(w, h\left(\mathbb{S}^{1}\right)\right) \leq \kappa / 40$. Thus $\operatorname{dist}(\gamma, \partial h(T)) \geq 39 \kappa / 40$.

Let $\iota: \mathbb{R}^{3+m} \rightarrow \mathbb{R}^{3+m}$ be a linear transformation $\iota(x)=-w_{0}+x / \kappa$ which maps $\gamma$ into $B^{3+m}(\eta(5))$. Then

$$
\iota(\gamma) \in \mathcal{J}\left(2 \eta(5), 1 /\left(1000 \sqrt{3+m} n_{0}\right), m ; n\right) .
$$

By Lemma 11.2, there exists an $L_{0}$-bilipschitz, therefore $\eta^{\prime}$-quasisymmetric, homeomorphism $\chi^{\prime}$ of $\mathbb{R}^{3+m}$ so that $\chi^{\prime}(\iota(\gamma))=\partial B^{2}(\operatorname{diam} \iota(\gamma)) \subset \mathbb{R}^{2} \times \mathbb{R}^{1+m}$, where $\eta^{\prime}$ depends only on $\eta(5), m$, and $n_{0}$. Then $\chi=(\operatorname{diam} \iota(\gamma))^{-1} \chi^{\prime} \circ \iota$ is also $\eta^{\prime}$-quasisymmetric. Since $n \leq n_{0}$ and $n_{0}$ depends only on $\eta$, we have that $\eta^{\prime}=\eta^{\prime}(m, \eta)$. The existence of the constant $\delta_{0}$ follows from quasisymmetry of $\chi$ and geometry of $\mathbb{B}^{2+m} \times \mathbb{S}^{1}$. 
Proposition 11.3. Let $M$ be a PL 3-manifold with boundary in $\mathbb{R}^{3}$. Suppose $h: \mathbb{B}^{2} \times \mathbb{S}^{1} \rightarrow \mathbb{R}^{3}$ is an $\eta$-quasisymmetric embedding with the properties that $h$ embeds $\{0\} \times \mathbb{S}^{1}$ into $\partial M$ and $h \mid\left(\{0\} \times \mathbb{S}^{1}\right)$ is null-homotopic in $M$. Let $T=$ $h\left(\mathbb{B}^{2} \times \mathbb{S}^{1}\right)$. Then there exist an $\eta^{\prime}$-quasisymmetric homeomorphism $\chi: \mathbb{R}^{3} \rightarrow \mathbb{R}^{3}$, $\eta^{\prime}=\eta^{\prime}(\eta)$, and a constant $\delta_{0}=\delta_{0}(\eta)>0$ so that

(1) $\chi(T)$ contains the tube $N^{3}\left(\partial \mathbb{B}^{2}, \delta_{0}\right)$ in $\mathbb{R}^{3}$,

$(1)^{\prime}$ in particular, $\partial \mathbb{B}^{2}+j \subset \chi(T)$ for $j \in\{0\} \times\left[-\delta_{0}, \delta_{0}\right] \subset \mathbb{R}^{2} \times \mathbb{R}$, and

(2) $\chi \circ h \mid\left(\{0\} \times \mathbb{S}^{1}\right)$ is homotopic to the identity map id $\mid \partial \mathbb{B}^{2}$ in $\chi(T)$.

Proof. Let $\alpha: \mathbb{S}^{1} \rightarrow M$ be the map $x \mapsto h(0, x)$. Then, by assumption, $\alpha$ is simple and null-homtopic in $M$. We show first that $\alpha$ is an unknot. It suffices to show that there exists an embedding $\tau: \mathbb{B}^{2} \rightarrow M$ for which $\tau \mid \partial \mathbb{B}^{2}=\alpha$.

Since $\alpha$ is null-homotopic, there exists an extension $\hat{\alpha}: \mathbb{B}^{2} \rightarrow M$ of $\alpha$. Since $M$ is a PL manifold with boundary, $\partial M$ has a collar in $M$; see Corollary 2.26 in [16]. Thus we may assume that $\partial \mathbb{B}^{2}$ has a neighborhood $A$ in $\mathbb{B}^{2}$ for which $\hat{\alpha} \mid A$ is an embedding and $\hat{\alpha}^{-1}(\hat{\alpha}(A))=A$. Thus the conditions of Dehn's Lemma (see, e.g., Chapter 4 of [13]) are satisfied and there exists an embedding $\tau: \mathbb{B}^{2} \rightarrow M$ so that $\tau \mid \partial \mathbb{B}^{2}=\alpha$.

To unknot quantitatively, we follow the proof of Proposition 11.1 almost verbatim. Let $\kappa=\operatorname{dist}\left(h\left(\mathbb{S}^{1}\right), \partial T\right)$ and $n_{0}(\eta)$ be as in the proof of Proposition 11.1. Then there exists a polygonal Jordan path

$$
\gamma=\left[w_{0}, w_{1}\right] \cup\left[w_{1}, w_{2}\right] \cup \cdots \cup\left[w_{n-1}, w_{0}\right]
$$

with vertices $w_{i} \in\left(\kappa /\left(1000 \sqrt{3+m} n_{0}\right)\right) \mathbb{Z}^{3+m}$ so that $\max _{w \in \gamma} \operatorname{dist}\left(w, h\left(\mathbb{S}^{1}\right)\right) \leq$ $\kappa / 20$ and $\operatorname{dist}(\gamma, \partial h(T)) \geq 19 \kappa / 20$. Therefore $\gamma$ is PL-isotopic to $h\left(\{0\} \times \mathbb{S}^{1}\right)$ in $h(T)$. We may now fix a scaled $L_{0}=L_{0}\left(\eta, m, n_{0}\right)$-bilipschitz, therefore $\eta^{\prime}$ quasisymmetric, homeomorphism $\chi: \mathbb{R}^{3} \rightarrow \mathbb{R}^{3}$ so that $\chi\left(h\left(\mathbb{S}^{1}\right)\right)=\partial \mathbb{B}^{2}$, as in the proof of Proposition 11.1. Conditions (1) and (2) in the statement now follow by quasisymmetry.

\section{Growth and a modulus estimate for walls}

The main result in this section is a lower bound on the conformal modulus of a $m$-wall family, which corresponds partly to the first claim of [12, Proposition 4.5].

Proposition 12.1. Suppose $\left(\mathbb{R}^{3} / G, \mathcal{X},(\mathcal{C}, \mathcal{A}, \mathcal{W}), \theta, d_{\lambda}\right)$ is a Semmes space. Let $k \geq 0$, let $\mathcal{Y}$ be a collection of cubes-with-handles in $\mathcal{C}\left(X_{k}\right)$ of positive genus, and let $Y$ be their union. Let $m \geq 0$. Then the conformal modulus of $m$-walls satisfies

$$
\operatorname{Mod}_{\frac{3+m}{1+m}}\left(\hat{\Sigma}^{m}(Y, \mathcal{X}, a)\right) \geq C\left((\# \mathcal{Y})\left(\frac{a}{\lambda^{k}}\right)^{m}\right)^{1-(3+m) /(1+m)}
$$

for every $a>0$ and a constant $C=C(\mathcal{C}, \mathcal{W}, \mathcal{A}, m)>0$. 
To obtain the estimate, we first fix a collection of cubes-with-handles $\mathcal{H}=$ $\left\{\mathrm{H}_{0}, \mathrm{H}_{1}, \ldots\right\}$, one for each genus, and a special family of longitudes in each $\mathrm{H}_{g}$ as follows.

Let $Q(x, r)=\left[x_{1}-r, x_{1}+r\right] \times\left[x_{2}-r, x_{2}+r\right] \subset \mathbb{R}^{2}$ for $x=\left(x_{1}, x_{2}\right) \in \mathbb{R}^{2}$ and $r>0$ and denote the origin of $\mathbb{R}^{2}$ by $\mathrm{O}$. Set $\mathrm{H}_{0}=Q(\mathrm{O}, 1) \times[0,1]$.

For each $g>0$, fix points $\left\{p_{1}, \ldots, p_{g}\right\}$ in $Q(\mathrm{O}, 1-1 /(10 g))$ having pairwise distances at least $1 /(20 g)$. Let $\Omega_{g}=Q(\mathrm{O}, 1) \backslash \cup_{i}\left(\operatorname{int} Q\left(p_{i}, 1 /(100 g)\right)\right)$ and $\mathrm{H}_{g}=$ $\Omega_{g} \times[0,1]$. Then $\mathrm{H}_{g}$ is a cube-with- $g$-handles. For every $0 \leq t \leq 1 /(100 g)$ and every $0<s<1$, fix a PL 1-cycle $\sigma_{t, s}^{g}$ in $\mathrm{H}_{g}$ having

$$
\left(\partial Q(\mathrm{O}, 1-t) \cup \partial Q\left(p_{1}, \frac{1}{100 g}+t\right) \cup \cdots \cup \partial Q\left(p_{g}, \frac{1}{100 g}+t\right)\right) \times\{s\}
$$

as a carrier.

Lemma 12.2. Given $g>0$, the 1-cycles $\sigma_{t, s}^{g}$ defined above are longitudes of $\mathbf{H}_{g}$ for all $0 \leq t \leq 1 /(100 \mathrm{~g})$ and $0<s<1$. Moreover, if $\omega$ is a 2-manifold in $\mathbb{B}^{2}$ and $\zeta:(\omega, \partial \omega) \rightarrow\left(\mathrm{H}_{g}, \partial \mathrm{H}_{g}\right)$ is virtually interior essential, then $\zeta(\omega) \cap\left|\sigma_{t, s}^{g}\right| \neq \emptyset$.

Proof. We denote $\Omega=\Omega_{g}, \mathrm{H}=\mathrm{H}_{g}=\Omega_{g} \times[0,1]$, and $\sigma_{t, s}=\sigma_{t, s}^{g}$.

To show that $\sigma_{t, s}$ is a longitude, let $\alpha: \mathbb{S}^{1} \rightarrow \partial \mathrm{H}$ be a meridian of $\mathrm{H}$ and $\phi:\left(\mathbb{B}^{2}, \partial \mathbb{B}^{2}\right) \rightarrow(\mathrm{H}, \alpha)$ a map. We claim that $\phi\left(\mathbb{B}^{2}\right) \cap\left|\sigma_{t, s}\right| \neq \emptyset$.

Consider first the case $t=0$. Suppose aiming at a contradiction that there is an $s \in(0,1)$ so that $\phi\left(\mathbb{B}^{2}\right) \cap\left|\sigma_{0, s}\right|=\emptyset$. After postcomposing $\phi$ with a homeomorphism from $\mathbf{H} \backslash\left|\sigma_{0, s}\right|$ onto $\mathrm{H} \backslash(\partial \Omega \times[0,1])$, we may assume that $\phi:\left(\mathbb{B}^{2}, \partial \mathbb{B}^{2}\right) \rightarrow(\mathrm{H}, \Omega \times$ $\{0,1\})$. Suppose that $\phi\left(\partial \mathbb{B}^{2}\right) \subset \Omega \times\{1\}$. Since $\phi$ is interior essential, $\phi\left(\partial \mathbb{B}^{2}\right)$ is not trivial in $\pi_{1}(\Omega \times\{1\})$. Hence $\phi\left(\partial \mathbb{B}^{2}\right)$ is not trivial in $\pi_{1}(\Omega \times[0,1])=\pi_{1}(\mathrm{H})$. Since $\phi\left(\mathbb{B}^{2}\right) \subset \mathrm{H}$, this is a contradiction.

We next prove the second statement in the lemma for $t=0$. Let $\zeta:(\omega, \partial \omega) \rightarrow$ $(\mathrm{H}, \partial \mathrm{H})$ be the given map. Since $\zeta$ is virtually interior essential, it has an extension $\zeta^{\prime}: D_{\omega} \rightarrow \mathrm{H}$ satisfying $\zeta^{\prime}\left(D_{\omega} \backslash \omega\right) \subset \partial \mathrm{H}$, where $D_{\omega}$ is the 2-cell in $\mathbb{B}^{2}$ with $\omega \subset D_{\omega}$ and $\partial D_{\omega} \subset \partial \omega$; see Section 9.4. After applying a homotopy to $\zeta^{\prime}$ which leaves $\zeta^{\prime} \mid \partial D_{\omega}$ fixed, we may assume that $\zeta^{\prime}\left(D_{\omega}\right) \cap \partial \mathrm{H}=\zeta^{\prime}\left(\partial D_{\omega}\right) \cap \partial \mathrm{H}$. Since $\zeta^{\prime}$ is interior essential, $\zeta^{\prime}\left(D_{\omega}\right) \cap\left|\sigma_{0, s}\right| \neq \emptyset$ for all $s \in(0,1)$. Since $\zeta^{\prime}\left(\partial D_{\omega}\right) \subset \zeta(\partial \omega)$, $\zeta(\partial \omega) \cap\left|\sigma_{0, s}\right| \neq \emptyset$. Since $\zeta\left|\partial \omega=\zeta^{\prime}\right| \partial \omega$, the claim follows.

We now verify $\phi\left(\mathbb{B}^{2}\right) \cap\left|\sigma_{t, s}\right| \neq \emptyset$ in the case $0<t \leq 1 /(100 g)$ for a given $s \in(0,1)$. Let $\Omega_{t}$ be the planar closed region with boundary $\left|\sigma_{t, 0}\right|$, and $\mathrm{H}_{t, s}=$ $\Omega_{t} \times[s / 2,(1+s) / 2]$ a cube-with- $g$-handles contained in $\mathrm{H}$. Note that $\left|\sigma_{t, s}\right| \subset \partial \mathrm{H}_{t, s}$. Since $\mathbf{H} \backslash \mathrm{H}_{t, s}$ is a regular neighborhood of $\partial \mathrm{H}$ in $\mathrm{H}, \phi^{-1} \mathrm{H}_{t, s}$ contains a component, say $\omega^{\prime}$, on which $\phi \mid \omega^{\prime}:\left(\omega^{\prime}, \partial \omega^{\prime}\right) \rightarrow\left(\mathrm{H}_{t, s}, \partial \mathrm{H}_{t, s}\right)$ is virtually interior essential. Then, by the argument above, $\phi\left(\omega^{\prime}\right) \cap\left|\sigma_{t, s}\right| \neq \emptyset$ and hence $\phi\left(\mathbb{B}^{2}\right) \cap\left|\sigma_{t, s}\right| \neq \emptyset$. This proves the claim.

The second statement in the case $t>0$ follows from the same argument for $t=0$.

Proof of Proposition 12.1. By passing to a bilipschitz equivalent metric if necessary, we may assume that $d_{\lambda}=d_{\theta}$, where $\theta$ is a $\lambda$-modular embedding $\mathbb{R}^{3} / G \rightarrow \mathbb{R}^{n}$. 
As a preliminary step, we fix for every $\mathrm{c}=\left(A_{\mathrm{c}}, B_{\mathrm{c}}\right) \in \mathcal{C}$, a PL-homeomorphism $\xi_{\mathrm{c}}: A_{\mathrm{c}} \rightarrow \mathrm{H}_{g_{\mathrm{c}}}$, where $g_{\mathrm{c}}$ is the genus of $A_{\mathrm{c}}$. Since $\mathcal{C}$ is finite, the mappings $\xi_{\mathrm{c}}$ are uniformly bilipschitz, and there exists $t_{\mathcal{C}} \in(0,1 /(100 g))$ so that

$$
\xi_{\mathrm{c}}\left(B_{\mathrm{c}}\right) \cap\left|\sigma_{t, s}^{g_{\mathrm{c}}}\right|=\emptyset
$$

for every $0 \leq t \leq t_{\mathcal{C}}$, every $0<s<1$, and $\mathrm{c} \in \mathcal{C}$.

We fix a special family of longitudes for each $\mathrm{H}_{g}$ in $\mathcal{H}$ and an induced family of longitudes on $\mathcal{X}$ as follows. For each $g>0$, let

$$
\Sigma\left(\mathrm{H}_{g}, \mathcal{H}\right)=\left\{\sigma_{t, s}^{g}: 0 \leq t \leq r_{\mathcal{C}}, 0<s<1\right\}
$$

and for $g=0$, define $\Sigma\left(\mathrm{H}_{0}, \mathcal{H}\right)=\emptyset$.

By Lemma 12.2, these 1-cycles are longitudes of $\mathrm{H}_{g}$. Define for every $H \in \mathcal{C}(\mathcal{X})$ an induced family of longitudes of $H$ by

$$
\Sigma(H, \mathcal{X}, \mathcal{H})=\left\{\varphi_{H}^{-1} \circ \xi_{\mathrm{c}_{H}}^{-1}(\sigma): \sigma \in \Sigma\left(\mathrm{H}_{g_{H}}, \mathcal{H}\right)\right\}
$$

where $g_{H}$ is the genus of $H$ and $\varphi_{H}: H^{\text {diff }} \rightarrow \mathrm{c}_{H}^{\text {diff }}$ is the chart map in $\mathcal{A}$.

By (9.2), every 1-cycle in $Y$ of the form

$$
\tau_{t, s}=\sum_{H \in \mathcal{Y}} \varphi_{H}^{-1} \circ \xi_{\mathrm{c}_{H}}^{-1}\left(\sigma_{t, s}^{g_{H}}\right)
$$

$0 \leq t \leq t_{\mathrm{C}}$ and $0<s<1$, is a longitude of $Y$. Set

$$
\Sigma(Y, \mathcal{X}, \mathcal{H})=\left\{\tau_{t, s}: 0 \leq t \leq t_{\mathcal{C}} \text { and } 0<s<1\right\}
$$

and

$$
\Sigma^{m}(Y, \mathcal{X}, \mathcal{H} ; a)=\left\{|\tau| \times[-a, a]^{m}: \tau \in \Sigma(Y, \mathcal{X}, \mathcal{H})\right\}
$$

the collection of corresponding $m$-walls over $Y$ of height $a$.

Since $\Sigma^{m}(Y, \mathcal{X}, \mathcal{H} ; a) \subset \Sigma^{m}(Y, \mathcal{X} ; a)$, it suffices to show that the estimate $(12.1)$ holds for the surface family $\hat{\Sigma}^{m}(Y, \mathcal{X}, \mathcal{H} ; a)=\left(\pi_{G} \times\right.$ id $)\left(\Sigma^{m}(Y, \mathcal{X}, \mathcal{H} ; a)\right)$.

Before continuing, we observe that, since the embedding $\theta: \mathbb{R}^{3} / G \rightarrow \mathbb{R}^{n}$ is $\lambda$-modular, there exists $L=L(\mathcal{C}, \mathcal{A}, \mathcal{W}) \geq 1$ so that for every $k \geq 0$ and every $H \in \mathcal{C}\left(X_{k}\right)$, the map

$$
\zeta_{H}=\pi_{G} \circ \varphi_{H}^{-1} \circ \xi_{\mathrm{c}_{H}}^{-1} \mid \xi_{\mathrm{c}_{H}}\left(\mathrm{c}_{H}^{\text {diff }}\right): \xi_{\mathrm{c}_{H}}\left(\mathrm{c}_{H}^{\text {diff }}\right) \rightarrow \pi_{G}\left(H^{\text {diff }}\right)
$$

and its extension $\xi_{\mathrm{c}_{H}}\left(\mathrm{c}_{H}^{\text {diff }}\right) \times \mathbb{R}^{m} \rightarrow\left(\pi_{G}(H) \times \mathbb{R}^{m}, d_{\lambda, m}\right)$ defined by

$$
\zeta_{H}:(x, z) \mapsto\left(\pi_{G} \circ \varphi_{H}^{-1} \circ \xi_{\mathrm{c}_{H}}^{-1}(x), \lambda^{k} z\right)
$$

are $\left(\lambda^{k}, L\right)$-quasisimilarities.

In the following estimation of the modulus of surface families, we denote by $\mathcal{H}_{\delta}^{\beta}$ and by $\mathcal{H}_{e}^{\beta}$ the $\beta$-dimensional Hausdorff measures with respect to $d_{\lambda, m}$ and the Euclidean metric, respectively. 
Suppose that $\rho$ is an admissible Borel function for $\hat{\Sigma}^{m}(Y, \mathcal{X}, \mathcal{H} ; a)$ on $\mathbb{R}^{3} / G \times$ $\mathbb{R}^{m}$, that is,

$$
\int_{\pi_{G}\left(\left|\tau_{t, s}\right|\right) \times[-a, a]^{m}} \rho \mathrm{d} \mathcal{H}_{\delta}^{1+m} \geq 1
$$

for every $\tau_{t, s} \in \Sigma(Y, \mathcal{X}, \mathcal{H})$. We assume, as we may, that $\rho$ is supported in $\pi_{G}(Y \backslash$ $\left.X_{k+1}\right) \times[-a, a]^{m}$.

We have, for every $0 \leq t \leq t_{e}$ and every $0<s<1$, that

$$
\begin{aligned}
& \sum_{H \in \mathcal{Y}}\left(L \lambda^{k}\right)^{1+m} \int_{\left|\sigma_{t, s}^{g_{H}}\right| \times\left[-\lambda^{-k} a, \lambda^{-k} a\right]^{m}} \rho \circ \zeta_{H} \mathrm{~d} \mathcal{H}_{e}^{1+m} \\
& \geq \sum_{H \in \mathcal{Y}} \int_{\zeta_{H}\left(\left|\sigma_{t, s}^{g_{H}}\right| \times\left[-\lambda^{-k} a, \lambda^{-k} a\right]^{m}\right)} \rho \mathrm{d} \mathcal{H}_{\delta}^{1+m}=\int_{\pi_{G}\left(\left|\tau_{t, s}\right|\right) \times[-a, a]^{m}} \rho \mathrm{d} \mathcal{H}_{\delta}^{1+m} \geq 1 .
\end{aligned}
$$

Thus

$$
\begin{aligned}
& \sum_{H \in \mathcal{Y}} \int_{\mathrm{H}_{g_{H}} \times\left[-\lambda^{-k} a, \lambda^{-k} a\right]^{m}} \rho \circ \zeta_{H} \mathrm{~d} \mathcal{H}_{e}^{3+m} \\
& \quad \geq C \int_{\left[0, t_{\mathcal{C}}\right] \times[0,1]}\left(\sum_{H \in \mathcal{Y}} \int_{\mid \sigma_{t, s}^{g_{H} \mid \times\left[-\lambda^{-k} a, \lambda^{-k} a\right]^{m}}} \rho \circ \zeta_{H} \mathrm{~d} \mathcal{H}_{e}^{1+m}\right) \mathrm{d} \mathcal{H}_{e}^{2} \\
& \quad \geq C t_{\mathrm{C}} \lambda^{-k(1+m)}
\end{aligned}
$$

where $C$ depends only $(\mathcal{C}, \mathcal{A}, \mathcal{W})$.

Let $p=(3+m) /(1+m)$. Then, by $(12.2)$,

$$
\begin{aligned}
\sum_{H \in \mathcal{Y}} & \int_{\mathrm{H}_{g_{H}} \times\left[-\lambda^{-k} a, \lambda^{-k} a\right]^{m}}\left(\rho \circ \zeta_{H}\right)^{p} \mathrm{~d} \mathcal{H}_{e}^{3+m} \\
\geq & \left(\sum_{H \in \mathcal{Y}} \mathcal{H}_{e}^{3+m}\left(\mathrm{H}_{g_{H}} \times\left[-\lambda^{-k} a, \lambda^{-k} a\right]^{m}\right)\right)^{1-p} \\
& \times\left(\sum_{H \in \mathcal{Y}} \int_{\mathrm{H}_{g_{H}} \times\left[-\lambda^{-k} a, \lambda^{-k} a\right]^{m}} \rho \circ \zeta_{H} \mathrm{~d} \mathcal{H}_{e}^{3+m}\right)^{p} \\
\geq & C(\# \mathcal{Y})^{1-p}\left(\lambda^{-k} a\right)^{m(1-p)} \lambda^{-k(1+m) p}=C(\# \mathcal{Y})^{1-p} \lambda^{-k(m+p)} a^{m(1-p)},
\end{aligned}
$$

where $C>0$ depends only on $m$ and $(\mathcal{C}, \mathcal{A}, \mathcal{W})$.

Since $\zeta_{H}$ is a $\left(\lambda^{k}, L\right)$-quasisimilarity, $\zeta_{H}^{-1}$ is $L \lambda^{-k}$-Lipschitz. By a change of variables,

$$
\begin{aligned}
\int_{\pi_{G}(H) \times[-a, a]^{m}} \rho^{p} \mathrm{~d} \mathcal{H}_{\delta}^{3+m} & =\int_{\pi_{G}(H) \times[-a, a]^{m}}\left(\rho \circ \zeta_{H}\right)^{p} \circ \zeta_{H}^{-1} \mathrm{~d} \mathcal{H}_{\delta}^{3+m} \\
& \geq\left(\frac{\lambda^{k}}{L}\right)^{3+m} \int_{\mathrm{H}_{g_{H}} \times\left[-\lambda^{-k} a, \lambda^{-k} a\right]^{m}}\left(\rho \circ \zeta_{H}\right)^{p} \mathrm{~d} \mathcal{H}_{e}^{3+m}
\end{aligned}
$$


for every $H \in \mathcal{Y}$. Since $\rho$ is supported in $\pi_{G}\left(Y \backslash X_{k+1}\right) \times[-a, a]^{m}$, we have

$$
\begin{array}{rl}
\int_{\mathbb{R}^{3} / G \times \mathbb{R}^{m}} \rho^{p} & \mathrm{~d} \mathcal{H}_{\delta}^{3+m}=\int_{\pi_{G}\left(Y \backslash X_{k+1}\right) \times[-a, a]^{m}} \rho^{p} \mathrm{~d} \mathcal{H}_{\delta}^{3+m} \\
\geq & \left(\lambda^{k} / L\right)^{3+m} \sum_{H \in \mathcal{Y}} \int_{\mathrm{H}_{g_{H}} \times\left[-\lambda^{-k} a, \lambda^{-k} a\right]^{m}}\left(\rho \circ \zeta_{H}\right)^{p} \mathrm{~d} \mathcal{H}_{e}^{3+m} \\
\geq C(\# \mathcal{Y})^{1-p} \lambda^{k(3+m)} a^{m(1-p)} \lambda^{-k(m+p)}=C\left((\# \mathcal{Y})\left(a / \lambda^{k}\right)^{m}\right)^{1-p},
\end{array}
$$

where $C$ depends only on $m$ and $(\mathcal{C}, \mathcal{A}, \mathcal{W})$. The claim follows.

\section{A necessary condition for quasisymmetric parametriza- tion}

The existence of a quasisymmetric parametrization of $\left(\mathbb{R}^{3} / G \times \mathbb{R}^{m}, d_{\lambda, m}\right)$ by $\mathbb{R}^{3+m}$ requires a balance among the growth, circulation and the scaling factor of the Semmes space. We prove this result in this section.

Theorem 13.1. Let $\left(\mathbb{R}^{3} / G, \mathcal{X},(\mathcal{C}, \mathcal{A}, \mathcal{W}), \theta, d_{\lambda}\right)$ be a Semmes space, and let $m \geq 0$. Assume that $\mathcal{X}$ has order of growth at most $\gamma$ and order of circulation at least $\omega$. Suppose that there exists a quasisymmetric homeomorphism $\left(\mathbb{R}^{3} / G \times \mathbb{R}^{m}, d_{\lambda, m}\right) \rightarrow$ $\mathbb{R}^{3+m}$. Then

$$
\lambda^{m} \omega^{(3+m) / 2} \leq \gamma
$$

We obtain now Theorem 1.3 as a corollary.

Proof of Theorem 1.3. Since $\omega^{3}>\gamma^{2} \geq 1$, we may fix $\lambda$ so that $\omega^{-1 / 2}<\lambda<\gamma^{-1 / 3}$. On the one hand, $\lambda \gamma^{3}<1$, so $\left(\mathbb{R}^{3} / G \times \mathbb{R}^{m}, d_{\lambda, m}\right)$ is Ahlfors $(3+m)$-regular for all $m \geq 0$. On the other hand,

$$
\lambda^{m} \omega^{(3+m) / 2}>\gamma
$$

so there are no quasisymmetric homeomorphisms $\left(\mathbb{R}^{3} / G \times \mathbb{R}^{m}, d_{\lambda, m}\right) \rightarrow \mathbb{R}^{3+m}$ for any $m \geq 0$. The linear local contractibility follows from Proposition 7.9.

To combine the modulus estimates in Sections 10 and 12, we need a one-sided comparison between the modulus of a wall family and the modulus of a quasisymmetric image of the same family. The proof of Proposition 4.1 in [12] for the case of the Whitehead continuum applies almost verbatim to the Semmes spaces $\mathbb{R}^{3} / G \times \mathbb{R}^{m} ;$ we omit the details.

Proposition 13.2. Suppose $f: \mathbb{R}^{3} / G \times \mathbb{R}^{m} \rightarrow \mathbb{R}^{3+m}$ is an $\eta$-quasisymmetric homeomorphism, and $Y$ is the union of a nonempty subcollection of cubes-withhandles in $\mathcal{C}\left(X_{k}\right)$ for some $k \geq 1$. Then there exists $C=C(\eta)>0$ so that, for $a>0$,

$$
\operatorname{Mod}_{\frac{3+m}{1+m}}\left(\hat{\Sigma}^{m}(Y, \mathcal{X}, a)\right) \leq C \operatorname{Mod}_{\frac{3+m}{1+m}} f\left(\hat{\Sigma}^{m}(Y, \mathcal{X}, a)\right) .
$$


Proof of Theorem 13.1. Let $\alpha_{0} \in \mathcal{M}(\mathcal{X})$ be a meridian as in Definition 9.2. In view of Lemma 9.3, when considering a lower bound for the circulation, we may restrict to a subcollection $\mathcal{M}$ of $\mathcal{M}_{\mathcal{A}}\left(\mathcal{X} ; \alpha_{0}\right)$ consisting of uniformly quasisimilar meridians.

Since the order of circulation of $\mathcal{X}$ is at least $\omega$, there exists $C>0$ so that for each $\ell>1$, there exist $k, k^{\prime} \geq 0, k^{\prime}-k \geq \ell, H \in \mathcal{C}\left(X_{k}\right)$, and $\alpha: \mathbb{S}^{1} \rightarrow \partial H$ in $\mathcal{M}$ so that

$$
\operatorname{circ}\left(X_{k^{\prime}} \cap H, \alpha, H\right) \geq C \omega^{k^{\prime}-k} .
$$

Let $f$ be an $\eta$-quasisymmetric mapping $\left(\mathbb{R}^{3} / G \times \mathbb{R}^{m}, d_{\lambda, m}\right) \rightarrow \mathbb{R}^{3+m}$. From Lemma 9.3, Theorem 10.1, Proposition 12.1, and Proposition 13.2 it follows that

$$
\left(\# \mathcal{C}\left(X_{k^{\prime}} \cap H\right)\left(A \lambda^{k}\right)^{m} \lambda^{-k^{\prime} m}\right)^{1-p} \leq C\left(\frac{1}{\operatorname{circ}\left(X_{k^{\prime}} \cap H, \alpha, H\right)}\right)^{p},
$$

where $p=(3+m) /(1+m) ; C>0$ depends only on $(\mathcal{C}, \mathcal{A}, \mathcal{W}), \lambda$, and $m$; and $A$ is the constant defined in Theorem 10.1. Since the order of growth of $\mathcal{X}$ is at most $\gamma$, there exists $C=C\left(\mathcal{C}, \mathcal{W}, \mathcal{A}, m, \eta, \alpha_{0}\right) \geq 1$ such that

$$
\begin{aligned}
\omega^{\left(k^{\prime}-k\right) p} & \leq C\left(\operatorname{circ}\left(X_{k^{\prime}} \cap H, \alpha, H\right)\right)^{p} \leq C\left(\# \mathcal{C}\left(X_{k^{\prime}} \cap H\right)\left(A \lambda^{k}\right)^{m} \lambda^{-k^{\prime} m}\right)^{p-1} \\
& \leq C\left(\gamma^{k^{\prime}-k} \lambda^{k m} \lambda^{-k^{\prime} m}\right)^{p-1} \leq C \lambda^{\left(k-k^{\prime}\right) m(p-1)} \gamma^{\left(k^{\prime}-k\right)(p-1)}
\end{aligned}
$$

as $\ell \rightarrow \infty$. Thus

$$
\lambda^{m} \omega^{p /(p-1)} \leq C^{1 /\left(k^{\prime}-k\right)} \gamma \leq C^{1 / \ell} \gamma
$$

as $\ell \rightarrow \infty$. The claim now follows.

\section{Local parametrizability}

In this section we consider a local version of Theorem 13.1 that compares growth and circulation in parallel along a sequence of blocks of $\left(X_{k}\right)$ targeting a point $x \in \pi_{G}\left(X_{\infty}\right)$. Theorem 14.3 below can be used to detect the quasisymmetric nonparametrizability of some Semmes spaces, undetected by Theorem 13.1.

Let $\left(\mathbb{R}^{3} / G, \mathcal{X},(\mathcal{C}, \mathcal{A}, \mathcal{W})\right)$ be a decomposition space of finite type. Given $x \in$ $\pi_{G}\left(X_{\infty}\right)$, we denote by $\left(H_{k}(x)\right)$ the unique sequence in $\mathcal{C}(\mathcal{X})$ for which $x \in$ $\pi_{G}\left(H_{k}(x)\right)$ and $H_{k}(x) \in \mathcal{C}\left(X_{k}\right)$ for every $k \geq 0$. We call $\left(H_{k}(x)\right)$ the branch of $\mathcal{X}$ at $x$.

We denote by $\mathcal{M}(\mathcal{X}, x)$ the collection of all meridians on the branch $\left(H_{k}(x)\right)$ of $\mathcal{X}$ at $x$. Given a meridian $\alpha_{0} \in \mathcal{M}(\mathcal{X}, x)$, we write

$$
\mathcal{M}_{\mathcal{A}}\left(\mathcal{X}, x ; \alpha_{0}\right)=\mathcal{M}_{\mathcal{A}}\left(\mathcal{X} ; \alpha_{0}\right) \cap \mathcal{M}(\mathcal{X}, x),
$$

where $\mathcal{M}_{\mathcal{A}}\left(\mathcal{X} ; \alpha_{0}\right)$ is the collection of meridians in $\mathcal{X}$ related to $\alpha_{0}$ by $\mathcal{A}$ defined in $(9.4)$.

Definition 14.1. At a point $x \in \pi_{G}\left(X_{\infty}\right)$, we say that the order of circulation of $\mathcal{X}$ is at least $\omega \geq 0$ and the order of growth of $\mathcal{X}$ is at most $\gamma \leq \infty$ concurrently if the following holds. There exists a meridian $\alpha_{0} \in \mathcal{M}(\mathcal{X}, x)$ and constants $C_{1}, C_{2}>0$ 
such that for every $\ell \geq 0$ there exist $k^{\prime}>k \geq 0$ with $k^{\prime}-k>\ell$ and a meridian $\alpha: \mathbb{S}^{1} \rightarrow \partial H_{k}(x)$ in $\mathcal{M}_{\mathcal{A}}\left(\mathcal{X}, x ; \alpha_{0}\right)$ satisfying

$$
\operatorname{circ}\left(X_{k^{\prime}} \cap H_{k}(x), \alpha, H_{k}(x)\right) \geq C_{1} \omega^{k^{\prime}-k},
$$

and

$$
\# \mathcal{C}\left(X_{k^{\prime}} \cap H_{k}(x)\right) \leq C_{2} \gamma^{k^{\prime}-k}
$$

Remark 14.2. By mixing the steps in the constructions of the Whitehead continuum and of Antoine's necklace, we may build a defining sequence $\mathcal{X}$ having the following property. Sequence $\mathcal{X}$ has order of growth at most $\gamma$ and order of circulation at least $\omega$ and, moreover, $\omega^{3}<\gamma^{2}$. Nevertheless, at each point $x \in \pi_{G}\left(X_{\infty}\right)$, a concurrent pair $(\omega(x), \gamma(x))$, as defined in 14.1 , may be chosen so that $\omega^{3}(x)>\gamma^{2}(x)$.

Theorem 14.3. Let $\left(\mathbb{R}^{3} / G, \mathcal{X},(\mathcal{C}, \mathcal{A}, \mathcal{W}), \theta, d_{\lambda}\right)$ be a Semmes space and let $x \in$ $\pi_{G}\left(X_{\infty}\right)$. Suppose at $x, \mathcal{X}$ has order of growth at most $\gamma(x)$ and order of circulation at least $\omega(x)$ concurrently. If for some $\delta>0$ and a neighborhood $U$ of $x$ there exists a quasisymmetric embedding $\left(U \times(-\delta, \delta)^{m}, d_{\lambda, m}\right) \rightarrow \mathbb{R}^{3+m}$ then

$$
\lambda^{m} \omega(x)^{(3+m) / 2} \leq \gamma(x) .
$$

Sketch of the proof. The only essential modification to the proof of Theorem 13.1 is related to the application of a local version of Theorem 10.1.

Let $U \subset \mathbb{R}^{3} / G$ be an open set containing $x$ and $f:\left(U \times(-\delta, \delta)^{m}, d_{\lambda, m}\right) \rightarrow$ $\mathbb{R}^{3+m}$ be a quasisymmetric embedding. We may fix a ball $B^{3+m}\left(f(x), r_{0}\right)$ in $f\left(U \times(-\delta, \delta)^{m}\right)$, and an integer $k_{0}>0$ so that $\pi_{G}\left(H_{k_{0}}(x)\right) \times\left[-\lambda^{k_{0}}, \lambda^{k_{0}}\right]^{m} \subset$ $U \times(-\delta, \delta)^{m}$. Under these choices of parameters, the quasisymmetric unknotting of images of meridians (Proposition 11.1 and Proposition 11.3) can be performed in $B^{3+m}\left(f(x), r_{0}\right)$. Thus the proof of Theorem 10.1 can be carried over to the defining sequence $\left(X_{k}\right)_{k \geq k_{0}}$. We omit the straightforward modifications of Theorem 10.1 and the related lemmas in Sections 10, 11, and 12.

\section{Singular fibers of Semmes spaces}

In this section, we consider an application of Theorem 14.3 to a question on the quasisymmetric equivalence of product spaces $\left(\mathbb{R}^{3} / G \times \mathbb{R}^{1}, d_{\lambda, 1}\right)$ for $0<\lambda<1$.

Let $\left(\mathbb{R}^{3} / G,\left(X_{k}\right), d_{\lambda}\right)$ be a Semmes space and $m \geq 0$. A point $x \in \mathbb{R}^{3} / G$ is said to be (quasisymmetrically) $\lambda$-singular of index $m$ if there is no quasisymmetric homeomorphism from any neighborhood of $(x, 0)$ in $\mathbb{R}^{3} / G \times \mathbb{R}^{m}$ to a subset of $\mathbb{R}^{3+m}$; in this case, $\{x\} \times \mathbb{R}^{m} \subset \mathbb{R}^{3} / G \times \mathbb{R}^{m}$ is called a singular fiber. We denote by $\operatorname{sing}_{\lambda, m}\left(\mathbb{R}^{3} / G\right)$ the set of $\lambda$-singular points of index $m$ and note that $\operatorname{sing}_{\lambda, m}\left(\mathbb{R}^{3} / G\right)$ is a closed subset of $\pi_{G}\left(X_{\infty}\right)$.

A quasisymmetric map $\left(\mathbb{R}^{3} / G \times \mathbb{R}^{m}, d_{\lambda, m}\right) \rightarrow\left(\mathbb{R}^{3} / G^{\prime} \times \mathbb{R}^{m}, d_{\mu, m}\right)$ between two Semmes spaces induces a homeomorphism from $\operatorname{sing}_{\lambda, m}\left(\mathbb{R}^{3} / G\right) \times \mathbb{R}^{m}$ to $\operatorname{sing}_{\mu, m}\left(\mathbb{R}^{3} / G^{\prime}\right) \times \mathbb{R}^{m}$. For $m=1$, the induced map is bilipschitz on nonisolated fibers. 
Theorem 15.1. Let $\left(\mathbb{R}^{3} / G,\left(X_{k}\right), d_{\lambda}\right)$ and $\left(\mathbb{R}^{3} / G^{\prime},\left(Y_{k}\right), d_{\mu}\right)$ be two Semmes spaces, let $m \geq 1$, and let $f:\left(\mathbb{R}^{3} / G \times \mathbb{R}, d_{\lambda, m}\right) \rightarrow\left(\mathbb{R}^{3} / G^{\prime} \times \mathbb{R}, d_{\mu, m}\right)$ be an $\eta$-quasisymmetric map. Then

$$
f\left(\operatorname{sing}_{\lambda, m}\left(\mathbb{R}^{n} / G\right) \times \mathbb{R}\right)=\operatorname{sing}_{\mu, m}\left(\mathbb{R}^{3} / G^{\prime}\right) \times \mathbb{R} .
$$

Furthermore, if $m=1$ and $A$ is the collection of accumulation points in $\operatorname{sing}_{\lambda, 1}\left(\mathbb{R}^{3} / G\right)$, then $f \mid A \times \mathbb{R}$ is $L_{0}$-bilipschitz for some $L_{0} \geq 1$.

By quasisymmetry, the bilipschitz rigidity of the singular fibers yields the nesting of corresponding branches. We formalize this observation in the next theorem. As an application of this result, we obtain the quasisymmetric inequivalence of $\left(\mathbb{R}^{3} / \mathrm{Bd} \times \mathbb{R}^{1}, d_{\lambda, 1}\right)$ and $\left(\mathbb{R}^{3} / \mathrm{Bd} \times \mathbb{R}^{1}, d_{\lambda^{\prime}, 1}\right)$ for $\lambda \neq \lambda^{\prime}$ and $1 / 2<\lambda^{\prime}<1$; see Theorem 1.5 in the introduction. We postpone this discussion to Section 17.

Theorem 15.2. Let $0<\lambda<\mu<1$, let $\left(\mathbb{R}^{3} / G,\left(X_{k}\right), d_{\lambda}\right)$ and $\left(\mathbb{R}^{3} / G^{\prime},\left(Y_{k}\right), d_{\mu}\right)$ be Semmes spaces, and let $f:\left(\mathbb{R}^{3} / G \times \mathbb{R}, d_{\lambda, 1}\right) \rightarrow\left(\mathbb{R}^{3} / G^{\prime} \times \mathbb{R}, d_{\mu, 1}\right)$ be an $\eta$ quasisymmetric map. Let $x \in \operatorname{sing}_{\lambda, 1}\left(\mathbb{R}^{3} / G\right)$ be an accumulation point. Then, for any $\ell>0$, there exists $k_{0}=k_{0}\left(\eta, d_{\lambda}, d_{\mu}, \ell\right)>0$ so that

$$
f\left(\pi_{G}\left(H_{k}(x)\right) \times \mathbb{R}\right) \subset \pi_{G^{\prime}}\left(H_{k+\ell}(y)\right) \times \mathbb{R}
$$

for all $k \geq k_{0}$, where $y=\operatorname{proj} f(x)$ is the image of $f(x)$ under the projection proj: $\mathbb{R}^{3} / G^{\prime} \times \mathbb{R} \rightarrow \mathbb{R}^{3} / G^{\prime}$, and $\left(H_{k}(x)\right)$ and $\left(H_{k}(y)\right)$ are the branches of $\mathcal{X}=\left(X_{k}\right)$ and $\mathcal{Y}=\left(Y_{k}\right)$ at $x$ and $y$ respectively.

We begin with some auxiliary results on lines in metric spaces. Let $(X, d)$ be a metric space. We say that $L \subset X$ is a line if $L$ is isometric to $\mathbb{R}$. We say that a line $L$ is parallel to a line $L^{\prime}$ if there exists $a>0$ so that $\operatorname{dist}\left(p, L^{\prime}\right)=a$ for every $p \in L$; in this case, $L^{\prime}$ is also parallel to $L$ and $\operatorname{dist}\left(L, L^{\prime}\right)=a$.

Lemma 15.3. Suppose $f: X \rightarrow Y$ is an $\eta$-quasisymmetric map between two metric spaces that maps two given parallel lines $L$ and $L^{\prime}$ to parallel lines $f L$ and $f L^{\prime}$. Then there exists $C=C(\eta)>1$ so that

$$
\frac{1}{C} \frac{\operatorname{dist}_{Y}\left(f L, f L^{\prime}\right)}{\operatorname{dist}_{X}\left(L, L^{\prime}\right)} \leq \frac{\operatorname{dist}_{Y}(f(p), f(q))}{\operatorname{dist}_{X}(p, q)} \leq C \frac{\operatorname{dist}_{Y}\left(f L, f L^{\prime}\right)}{\operatorname{dist}_{X}\left(L, L^{\prime}\right)}
$$

for all $p, q \in L$ with $\operatorname{dist}_{X}(p, q) \geq \operatorname{dist}_{X}\left(L, L^{\prime}\right)$.

Proof. Suppose that the points $p, q \in L$ have distance $\operatorname{dist}_{X}(p, q) \geq \operatorname{dist}_{X}\left(L, L^{\prime}\right)$. Since $L$ is a line, there exist points $p=p_{0}, \ldots, p_{k}=q$ on $L$ so that

$$
\operatorname{dist}_{X}\left(L, L^{\prime}\right) \leq \operatorname{dist}_{X}\left(p_{i}, p_{i-1}\right) \leq 2 \operatorname{dist}_{X}\left(L, L^{\prime}\right)
$$

for all $1 \leq i \leq k$. Since the lines $L$ and $L^{\prime}$ are parallel, the lines $f L$ and $f L^{\prime}$ are parallel, and $f$ is $\eta$-quasisymmetric, there exists $C_{0}=C_{0}(\eta)>1$ so that

$$
\frac{1}{C_{0}} \operatorname{dist}_{Y}\left(f L, f L^{\prime}\right) \leq \operatorname{dist}_{Y}\left(f\left(p_{i}\right), f\left(p_{i-1}\right)\right) \leq C_{0} \operatorname{dist}_{Y}\left(f L, f L^{\prime}\right)
$$

for all $1 \leq i \leq k$. Since $L$ and $f L$ are lines, the claim follows by summing. 
Corollary 15.4. Let $f: X \rightarrow Y$ be an $\eta$-quasisymmetric map between two metric spaces that maps two given parallel lines $L$ and $L^{\prime}$ to two parallel lines $f L$ and $f L^{\prime}$. Suppose, in addition, that $f$ maps a sequence $\left(L_{i}\right)$ of lines parallel to $L$ tending to $L$ to a sequence $\left(f L_{i}\right)$ of lines parallel to $f L$ tending to $f L$. Then there exists $C=C(\eta)>1$ so that

$$
\frac{1}{C} \frac{\operatorname{dist}_{Y}\left(f L, f L^{\prime}\right)}{\operatorname{dist}_{X}\left(L, L^{\prime}\right)} \leq \frac{\operatorname{dist}_{Y}(f(p), f(q))}{\operatorname{dist}_{X}(p, q)} \leq C \frac{\operatorname{dist}_{Y}\left(f L, f L^{\prime}\right)}{\operatorname{dist}_{X}\left(L, L^{\prime}\right)}
$$

for $p, q \in L$.

Proof. Calculations using Lemma 15.3 show that there exists a constant $C>1$ so that

$$
\frac{1}{C} \frac{\operatorname{dist}_{Y}\left(f L, f L^{\prime}\right)}{\operatorname{dist}_{X}\left(L, L^{\prime}\right)} \leq \frac{\operatorname{dist}_{Y}\left(f L, f L_{i}\right)}{\operatorname{dist}_{X}\left(L, L_{i}\right)} \leq C \frac{\operatorname{dist}_{Y}\left(f L, f L^{\prime}\right)}{\operatorname{dist}_{X}\left(L, L^{\prime}\right)}
$$

for every $i \geq 0$.

Given $p, q \in L$, we fix a line $L_{i}$ so that $\operatorname{dist}_{X}\left(L, L_{i}\right)<\operatorname{dist}_{X}(p, q)$. The claim now follows by applying Lemma 15.3 again.

Proof of Theorem 15.1. The first claim is clear.

Suppose next that $f: \mathbb{R}^{3} / G \times \mathbb{R} \rightarrow \mathbb{R}^{3} / G^{\prime} \times \mathbb{R}$ is quasisymmetric and $x \in$ $\operatorname{sing}_{\lambda, 1}\left(\mathbb{R}^{3} / G\right)$ is an accumulation point. We choose a point $x^{\prime}$ in $\operatorname{sing}_{\lambda, 1}\left(\mathbb{R}^{3} / G\right)$ so that $d_{\lambda, 1}\left(x, x^{\prime}\right) \geq \frac{1}{2}$ diam $\operatorname{sing}_{\lambda, 1}\left(\mathbb{R}^{3} / G\right)$, and let $L$ and $L^{\prime}$ be the singular fibers $\{x\} \times \mathbb{R}$ and $\left\{x^{\prime}\right\} \times \mathbb{R}$, respectively. In view of Corollary 15.4,

$$
\frac{1}{C_{0}} \leq \frac{\operatorname{dist}_{\mu, 1}(f(p), f(q))}{\operatorname{dist}_{\lambda, 1}(p, q)} \leq C_{0}
$$

for all $p=(x, s)$ and $q=(x, t)$ in the singular fiber $\{x\} \times \mathbb{R}$, where $C_{0}>1$ depends only on the data and not on $x$.

Let $p=(x, s)$ and $w=(y, r) \in \operatorname{sing}_{\lambda, 1}\left(\mathbb{R}^{3} / G\right) \times \mathbb{R}$, and set $q=(x, t)$, where $t$ is defined by $t=r+d_{\lambda, 1}(x, y)$ if $r \geq s$ and by $t=r-d_{\lambda, 1}(x, y)$ if $r<s$. So, $\operatorname{dist}_{\lambda, 1}(p, w)=\operatorname{dist}_{\lambda, 1}(p, q)$. By $\eta$-quasisymmetry,

$$
\frac{1}{\eta(1)} \leq \frac{\operatorname{dist}_{\mu, 1}(f(p), f(w))}{\operatorname{dist}_{\mu, 1}(f(p), f(q))} \leq \eta(1) .
$$

Hence

$$
\frac{1}{C_{0} \eta(1)} \leq \frac{\operatorname{dist}_{\mu, 1}(f(p), f(w))}{\operatorname{dist}_{\lambda, 1}(p, w)} \leq C_{0} \eta(1) .
$$

The second claim now follows.

Proof of Theorem 15.2. By properties of the Semmes metric (see Section 7.1), there exist $C_{1}>1$ and $C_{2}>1$ so that

$$
\operatorname{diam}_{d_{\lambda, 1}}\left(\pi_{G}(T)\right) \leq C_{1} \lambda^{\operatorname{level}(T)}
$$


and

$$
\operatorname{dist}_{d_{\mu, 1}}\left(\operatorname{sing}_{\mu, 1}\left(\mathbb{R}^{3} / G^{\prime}\right) \cap \pi_{G^{\prime}}\left(T^{\prime}\right), \partial \pi_{G^{\prime}}\left(T^{\prime}\right)\right) \geq \frac{1}{C_{2}} \mu^{\text {level }\left(T^{\prime}\right)}
$$

for every $T \in \mathcal{C}(\mathcal{X})$ and $T^{\prime} \in \mathcal{C}(\mathcal{Y})$.

Let $L_{0} \geq 1$ be the constant in Theorem 15.1. Since $\lambda<\mu$, we may fix $\ell>0$ and $k_{0}>0$ so that

$$
L_{0} C_{1} C_{2} \eta(1) \lambda^{k}<\mu^{k+\ell}
$$

for $k \geq k_{0}$.

Since $f(\{x\} \times \mathbb{R})=\{y\} \times \mathbb{R}, f(\{x\} \times \mathbb{R}) \subset f\left(\pi_{G}\left(H_{k}(x)\right) \times \mathbb{R}\right) \cap\left(\pi_{G^{\prime}}\left(H_{k^{\prime}}(y)\right) \times \mathbb{R}\right)$ for $k, k^{\prime} \geq 1$. From Theorem 15.1 and the $\eta$-quasisymmetry, it follows that for any $k \geq k_{0}$,

$$
\begin{aligned}
\operatorname{dist}_{\mathcal{H}}( & \left.f\left(\partial \pi_{G}\left(H_{k}(x)\right) \times \mathbb{R}\right), f(\{x\} \times \mathbb{R})\right) \leq L_{0} \eta(1) \operatorname{diam}_{d_{\lambda, 1}} \pi_{G}\left(H_{k}(x)\right) \\
& \leq L_{0} C_{1} \eta(1) \lambda^{k} \leq \mu^{k+\ell} / C_{2} \leq \operatorname{dist}_{d_{\lambda, 1}}\left(\partial \pi_{G^{\prime}}\left(H_{k+\ell}(y)\right) \times \mathbb{R},\{y\} \times \mathbb{R}\right),
\end{aligned}
$$

where $\operatorname{dist}_{\mathcal{H}}\left(f\left(\partial \pi_{G}\left(H_{k}(x)\right) \times \mathbb{R}\right), f(\{x\} \times \mathbb{R})\right)$ is the Hausdorff distance between $f\left(\partial \pi_{G}\left(H_{k}(x)\right) \times \mathbb{R}\right)$ and $f(\{x\} \times \mathbb{R})$ in the Semmes metric $d_{\mu, 1}$. Thus

$$
f\left(\pi_{G}\left(H_{k}(x)\right) \times \mathbb{R}\right) \subset \pi_{G^{\prime}}\left(H_{k+\ell}(y)\right) \times \mathbb{R} .
$$

This concludes the proof.

\section{Necklaces}

As an application of Theorem 8.2 we prove the existence of quasisymmetric parametrization for decomposition spaces associated with an Antoine necklace when the chains are long. For the statement, we introduce some terminology.

Let $I \geq 3$. A union $\bigcup_{i=1}^{I} T_{i}$ of pairwise disjoint tori $T_{1}, \ldots, T_{I}$ in $\mathbb{R}^{3}$ is called a chain if $T_{i} \cup T_{j}$ is a Hopf link if $|i-j|=1$ or $\{i, j\}=\{1, I\}$, and an unlink otherwise.

Suppose $T$ a torus in $\mathbb{R}^{3}$ and $\bigcup_{i=1}^{I} T_{i}$ is a torus chain contained in int $T$ in such a way that there is a homeomorphism $h: T \rightarrow \mathbb{B}^{2} \times \mathbb{S}^{1}$ satisfying $h(\partial T)=\partial \mathbb{B}^{2} \times \mathbb{S}^{1}$ and having the property that arguments of $p\left(h\left(T_{i}\right)\right)$ are contained, for each $i=$ $1, \ldots, I$, in $[2 \pi i / I, 2 \pi(i+4 / 3) / I]$. Here $p: \mathbb{B}^{2} \times \mathbb{S}^{1} \rightarrow \mathbb{S}^{1}$ is the projection map $(x, s) \mapsto s$. In this case, we say $\bigcup_{i=1}^{I} T_{i}$ is a necklace chain in $T$.

Let $\phi_{i}: U \rightarrow U_{i}$ be PL-homeomorphisms from a neighborhood $U$ of $T$ onto mutually disjoint neighborhoods $U_{i}$ of $T_{i}, 1 \leq i \leq I$, satisfying $T_{i} \subset U_{i} \subset T \subset U$. The initial package $\left(T, T_{1}, \ldots, T_{I} ; \phi_{1}, \ldots, \phi_{I}\right)$ yields a defining sequence $\mathcal{X}=\left(X_{k}\right)$ and a decomposition space, called an Antoine's $I$-necklace space, $\mathbb{R}^{3} / G$; see Section 4.2. It is easy to see that the diameters of components of $X_{k}$ can be arranged to tend to zero. Thus the components of $X_{\infty}$ are singletons and $\mathbb{R}^{3} / G$ is homeomorphic to $\mathbb{R}^{3}$.

As discussed in Section 4.2, the initial package induces a welding structure for the $I$-necklace space $\mathbb{R}^{3} / G$, therefore for each $\lambda>0$, a modular embedding of $\mathbb{R}^{3} / G$ 
and a Semmes metric $d_{\lambda}$ on $\mathbb{R}^{3} / G$. The Semmes spaces $\left(\mathbb{R}^{3} / G, d_{\lambda}\right)$ associated with necklaces are linearly locally contractible because the tori $T_{i}$ are contractible in $T$, and these spaces are Ahlfors 3-regular when $\lambda^{3} I<1$.

The existence of a quasisymmetric parametrization is proved in the following.

Theorem 16.1. For every $I \geq 10$, there exists a Semmes metric $d$ on the decomposition space $\mathbb{R}^{3} / G$ associated to an Antoine $I$-necklace so that $\left(\mathbb{R}^{3} / G, d\right)$ is quasisymmetric to $\mathbb{R}^{3}$.

The proof of Theorem 16.1 relies on the possibility of fitting a necklace chain of length $I$ in a torus, using only tori all similar to the larger one; we find it easier to fit a rectangular chain in a rectangular torus than to fit a round chain in a round torus.

16.0.1. Rectangular necklaces. Let $0<\lambda<b<a$. We define

$$
\begin{aligned}
& R_{+}(a, b, \lambda)=\left[-\frac{\lambda}{2}, a+\frac{\lambda}{2}\right] \times\left[-\frac{\lambda}{2}, b+\frac{\lambda}{2}\right], \\
& R_{-}(a, b, \lambda)=\left(\frac{\lambda}{2}, a-\frac{\lambda}{2}\right) \times\left(\frac{\lambda}{2}, b-\frac{\lambda}{2}\right),
\end{aligned}
$$

and

$$
T(a, b, \lambda)=\left(R_{+}(a, b, \lambda) \backslash R_{-}(a, b, \lambda)\right) \times\left[-\frac{\lambda}{2}, \frac{\lambda}{2}\right] .
$$

Let $L(a, b)=\partial([0, a] \times[0, b]) \times\{0\}$ be the boundary of the rectangle $[0, a] \times$ $[0, b] \times\{0\}$. We say $T(a, b, \lambda)$ is a torus with length $a+\lambda$, width $b+\lambda$, thickness $\lambda$, and core $L(a, b)$.

Let $T=T(a, b, \lambda)$. We say that components of

$$
\partial T \cap(\mathbb{R} \times\{-\lambda / 2, b+\lambda / 2\} \times \mathbb{R}) \text { and } \partial T \cap\left(\{-\lambda / 2, a+\lambda / 2\} \times \mathbb{R}^{2}\right)
$$

are the long and short faces of $T$, respectively. We call the components of

$$
\partial T \cap\left(\mathbb{R}^{2} \times\{-\lambda / 2, \lambda / 2\}\right)
$$

the boundary annuli of $T$.

We call the 3-cells

$\left[-\frac{\lambda}{2}, a+\frac{\lambda}{2}\right] \times\left[-\frac{\lambda}{2}, \frac{\lambda}{2}\right] \times\left[-\frac{\lambda}{2}, \frac{\lambda}{2}\right]$ and $\left[-\frac{\lambda}{2}, a+\frac{\lambda}{2}\right] \times\left[b-\frac{\lambda}{2}, b+\frac{\lambda}{2}\right] \times\left[-\frac{\lambda}{2}, \frac{\lambda}{2}\right]$ the long sides (front and back) of $T$, and similarly $\left[-\frac{\lambda}{2}, \frac{\lambda}{2}\right] \times\left[-\frac{\lambda}{2}, b+\frac{\lambda}{2}\right] \times\left[-\frac{\lambda}{2}, \frac{\lambda}{2}\right]$ and $\left[a-\frac{\lambda}{2}, a+\frac{\lambda}{2}\right] \times\left[-\frac{\lambda}{2}, b+\frac{\lambda}{2}\right] \times\left[-\frac{\lambda}{2}, \frac{\lambda}{2}\right]$ the short sides (left and right) of $T$.

We say that a torus $T$ in $\mathbb{R}^{3}$ is a rectangular torus if there exist a similarity $g: \mathbb{R}^{3} \rightarrow \mathbb{R}^{3}$ and $0<\lambda<b<a$ so that $T=g(T(a, b, \lambda))$. Furthermore, $T$ is 
$(p, q, r)$-oriented if $g=h \circ O$, where $h$ is a similarity of the form $x \mapsto \mu x+v, \mu>0$, and $O$ is an orthogonal transformation taking the standard basis $\left(e_{1}, e_{2}, e_{3}\right)$ to $\left(e_{p}, e_{q}, e_{r}\right)$. We call the images of the long (resp. short) sides (resp. faces) of $T(a, b, \lambda)$ the long (resp short) sides (resp. faces) of $T$.

In what follows we use the following three types of tightly fitted torus pairs. Let $T=T(A, B, 1)$ and let $T^{\prime}=g(T(a, b, \lambda))$ be an oriented torus contained in $T$. We say that $T^{\prime}$ is tightly fitted in $T$ if one of the following conditions holds:

1. $T^{\prime}$ is a $(1,2,3)$-oriented torus contained in a long side of $T$, so that each long face of $T^{\prime}$ intersects $\partial T$;

2. $T^{\prime}$ is a $(1,3,2)$-oriented torus contained in a long side of $T$, so that the long faces of $T^{\prime}$ are contained in the boundary annuli of $T$;

3. $T^{\prime}$ is a $(2,1,3)$-oriented torus contained in a short side of $T$, so that each long face of $T^{\prime}$ intersects $\partial T$ and the short faces of $T^{\prime}$ are contained in the long faces of $T$.

If $T^{\prime}$ is either a $(1,2,3)$ or $(1,3,2)$-oriented torus, we have the relations

$$
a+\lambda \leq A+1, \quad b+\lambda=1, \quad \text { and } \quad 2 \lambda<1 .
$$

If $T^{\prime}$ is $(2,1,3)$-oriented,

$$
a+\lambda=B+1, \quad b+\lambda=1, \quad \text { and } \quad 2 \lambda<1 .
$$

Proposition 16.2. Suppose $I \geq 10$. There exist $A>B>1$ and $a_{i}>b_{i}>$ $\lambda_{i},(1 \leq i \leq I)$ satisfying

$$
\frac{a_{i}}{A}=\frac{b_{i}}{B}=\frac{\lambda_{i}}{1}
$$

and the tori $T$ and $T_{i}, 1 \leq i \leq I$, are congruent to $T(A, B, 1)$ and $T\left(a_{i}, b_{i}, \lambda_{i}\right)$, respectively, such that the union $\bigcup_{1 \leq i \leq I} T_{i}$ is a necklace chain in $T$.

Theorem 16.1 now readily follows from this proposition and Theorem 8.2.

Proof of Theorem 16.1. Let $I \geq 10$ and let $T, T_{1}, \ldots, T_{I}$ be the tori constructed in Proposition 16.2. Let $\phi_{i}: \mathbb{R}^{3} \rightarrow \mathbb{R}^{3}$ be similarity maps $x \mapsto \lambda_{i} x+v_{i}$ so that $\phi_{i}(T)=T_{i}$ for $1 \leq i \leq I$. Then the initial package $\left(T, T_{1}, \ldots, T_{I}, \phi_{1}, \ldots, \phi_{I}\right)$ gives rise to a natural self-similar welding structure in $\mathbb{R}^{3}$ as in Section 4.2. The claim now follows from Theorem 8.2.

Proof. We construct for each $I \geq 10$, a torus $T=T(A, B, 1)$ and a chain $\bigcup_{1 \leq i \leq I} T_{i}$ which consists of tori all similar to $T$ and is tightly fitted in $T$.

Since the tori in the chain are pairwise disjoint, there exist similarity maps $h_{i}: \mathbb{R}^{3} \rightarrow \mathbb{R}^{3}, x \mapsto \mu x+v_{i}$, with $\mu \in(0,1)$ and $v_{i} \in \mathbb{R}^{3}$, so that the new chain $\bigcup_{i=1}^{I} h_{i}\left(T_{i}\right)$ is contained in the interior of $T$. Hence tori $h_{1}\left(T_{1}\right), \ldots, h_{I}\left(T_{I}\right)$ satisfy the claims of the proposition.

It remains to construct tori $T, T_{1}, \ldots, T_{I}$ with aforementioned properties. 
Case I. Suppose $I=4 k \geq 12$. Then $I=2 K+2$ for some odd integer $K \geq 5$. We seek $A>B>2$ and $a>b>2 \lambda$ satisfying

$$
\frac{a}{A}=\frac{b}{B}=\frac{\lambda}{1}
$$

and mutually disjoint tori $T_{i}, 1 \leq i \leq 2 K+2$, which are congruent to $T(a, b, \lambda)$ and contained in $T(A, B, 1)$ so that $\bigcup T_{i}$ forms a necklace-chain positioned as follows.

Tori $T_{1}$ and $T_{K+2}$ are $(2,1,3)$-oriented tori tightly fitted in the two short sides (left and right) of $T(A, B, 1)$ with cores lying on the plane $\left\{x_{3}=0\right\}$. The tori $T_{i}$ are $(1,3,2)$-oriented for even $i$, and $T_{i}$ are $(1,2,3)$-oriented for odd indices $i \neq 1, K+2$.

The tori $T_{2}, T_{3}, \ldots, T_{K+1}$ are tightly fitted in the front side of $T(A, B, 1)$, with the cores of $T_{2}, T_{4}, \ldots, T_{K+1}$ lying in the plane $\left\{x_{2}=0\right\}$ and the cores of $T_{3}, T_{5}, \ldots, T_{K}$ lying in $\left\{x_{3}=0\right\}$. The tori $T_{K+3}, T_{K+4}, \ldots, T_{2 K+2}$ are tightly fitted in the back side of $T(A, B, 1)$, with the cores of $T_{K+3}, T_{K+5}, \ldots, T_{2 K+1}$ lying in the plane $\left\{x_{2}=B\right\}$ and the cores of $T_{K+4}, T_{K+4}, \ldots, T_{2 K}$ lying in the plane $\left\{x_{3}=0\right\}$.

Since the necklace-chain $\bigcup_{i=1}^{I} T_{i}$ is tightly fitted in $T(A, B, 1)$,

$$
a+\lambda=B+1 \text { and } b+\lambda=1 .
$$

Since the tori $T_{1}$ and $T_{2}$, of thickness $\lambda$, are linked,

$$
3 \lambda<1 \text {. }
$$

In order to fit the linked chain $T_{1} \cup T_{2} \cup \ldots \cup T_{K+2}$ in a long side of $T(A, B, 1)$, we seek $\epsilon$ and $\delta$ in $(0,1 / 10)$ so that

$$
\begin{gathered}
A+1=K(a+\lambda)-(K-1)(2+\epsilon) \lambda+2(1+\delta) \lambda, \\
a+\lambda>2(2+\epsilon) \lambda, \\
1>(3+\delta) \lambda .
\end{gathered}
$$

Note that $K(a+\lambda)-(K-1)(2+\epsilon) \lambda$ is the total length of the union $T_{2} \cup T_{3} \cup \cdots \cup$ $T_{K+1}$, with $(K-1)(2+\epsilon) \lambda$ measuring the $K-1$ overlaps and $(1+\delta) \lambda$ measuring the distance from the chain to either short face of $T(A, B, 1)$. Conditions (16.7) and (16.8) are imposed to allow room for linking between consecutive tori in the union $T_{1} \cup T_{2} \cup \cdots \cup T_{K+2}$.

We now check that (16.3) to (16.8) can be realized with proper choices of $A, B, a, b, \lambda, \epsilon$, and $\delta$. By (16.3) and (16.4), we have the relations

$$
A+1=\lambda^{-2} \text { and } B+1=a+\lambda=\lambda^{-1} .
$$

Furthermore, by (16.6) and (16.9),

$$
2(K-2) \lambda^{3}-(2 \delta-(K-1) \epsilon) \lambda^{3}-K \lambda+1=0 .
$$

Let $0<\epsilon<1 /(5 K)$, to be fixed later, and fix $\delta=(K-1) \epsilon / 2$. Then $(16.10)$ becomes

$$
2(K-2) \lambda^{3}-K \lambda+1=0 .
$$


It is now easy to check that (16.11) admits a solution $\lambda \in(0,3 / 10)$. We now choose $\epsilon$ so small that (16.7) and (16.8) hold. The parameters $A, B, a$, and $b$ are now uniquely determined by (16.9) and (16.3).

Case II. Suppose that $I=4 k+2 \geq 10$. Then $I=2 K+2$ for an even $K \geq 4$. Again we will fit a necklace-chain $\bigcup_{i=1}^{2 K+2} T_{i}$, consisting of tori all similar to $T(A, B, 1)$, in the torus $T(A, B, 1)$.

Since $K$ is even, the linking condition forces $T_{1}$ and $T_{K+2}$ to have different $(p, q, r)$-orientations and unequal sizes. Let $T_{1}$ be a $(2,1,3)$-oriented torus tightly fitted in the left side of $T(A, B, 1)$ with the core lying on the plane $\left\{x_{3}=0\right\}$, and let $T_{K+2}$ be a smaller $(2,3,1)$-oriented torus (not tightly fitted) in the right side of $T(A, B, 1)$ with its core lying on the 2-plane $\left\{x_{1}=A\right\}$.

As in Case I, we choose $T_{i}$ to be $(1,3,2)$-oriented when $i \neq K+2$ is even and $T_{i}$ to be $(1,2,3)$-oriented when $i \neq 1$ is odd. The tori $T_{2}, T_{3}, \ldots, T_{K+1}$ will be tightly fitted in the front side of $T(A, B, 1)$ with the cores of $T_{2}, T_{4}, \ldots, T_{K}$ lying on the plane $\left\{x_{2}=0\right\}$ and the cores of $T_{3}, T_{5}, \ldots, T_{K+1}$ lying on $\left\{x_{3}=0\right\}$. Tori $T_{K+3}, T_{K+4}, \ldots, T_{2 K+2}$ will be tightly fitted in the back side of $T(A, B, 1)$ with the cores of $T_{K+3}, T_{K+5}, \ldots, T_{2 K+1}$ lying on the plane $\left\{x_{2}=B\right\}$ and cores of $T_{K+4}, T_{K+6}, \ldots, T_{2 K+2}$ lying on the plane $\left\{x_{3}=0\right\}$. Furthermore, one short face of $T_{K+1}$ and one short face of $T_{K+3}$ are placed in a common short face of $T(A, B, 1)$.

The tori $T_{i}, 1 \leq i \leq 2 K+2$ and $i \neq K+2$, are congruent to $T(a, b, \lambda)$ and the torus $T_{K+2}$ is congruent to a smaller $T\left(a^{\prime}, b^{\prime}, \lambda^{\prime}\right)$; all are similar to $T(A, B, 1)$.

It is straightforward to check that numbers $A>B>1, a>b>\lambda>0$, and $a^{\prime}>b^{\prime}>\lambda^{\prime}>0$ can be found so that $\bigcup_{i=1}^{I} T_{i}$ is a chain tightly fitted in $T$. We omit the details.

Case III. Suppose that $I \geq 11$ is odd. Then $I=2 K+3$ for some $K \geq 4$. For $K$ even, there exist, by Case I, numbers $A, B, a, b$, and $\lambda$ and tightly fitted tori $T_{1}, \ldots, T_{2 K+2}$ in $T=T(A, B, 1)$ so that tori $T_{1}, \ldots, T_{2 K+2}$ are congruent to $T(a, b, \lambda)$. For $K$ odd, we have, in addition, parameters $a^{\prime}, b^{\prime}$, and $\lambda^{\prime}$ so that the tori $T_{1}, \ldots, T_{2 K+2}$ are congruent to either $T(a, b, \lambda)$ or $T\left(a^{\prime}, b^{\prime}, \lambda^{\prime}\right)$. Let $\epsilon>0$ and $\delta>0$ be the parameters appearing in these constructions. We rename the first torus $T_{1}$ as $T_{0}$.

The plan is to replace the tori $T_{2}, T_{3}, T_{4}$ congruent to $T(a, b, \lambda)$ by four tori $t_{1}$, $t_{2}, t_{3}, t_{4}$ congruent to a smaller torus $T\left(a^{\prime \prime}, b^{\prime \prime}, \lambda^{\prime \prime}\right)$ which is similar to $T(a, b, \lambda)$. The new collection $T_{0}, t_{1}, t_{2}, t_{3}, t_{4}, T_{5}, \ldots, T_{2 K+2}$ forms the necklace chain for the case $I=2 K+3$.

Denote by $F_{\theta}$ the rotation in $\mathbb{R}^{3}$ about the $x_{1}$-axis by an angle $\theta$, so that $F_{\theta}\left(\mathbb{R}^{2} \times\{0\}\right)=P_{\theta}$, where $P_{\theta}$ is the plane $\left\{x_{3}=x_{2} \tan \theta\right\}$ in $\mathbb{R}^{3}$. Recall that $T_{0}$ is a $(2,1,3)$-torus and $T_{5}$ is a $(1,2,3)$-torus with cores lying on the plane $P_{0}$.

For $j=1, \ldots, 4$, let $t_{j}$ be a translate of $F_{2 j \pi / 5}\left(T\left(a^{\prime \prime}, b^{\prime \prime}, \lambda^{\prime \prime}\right)\right)$ in the direction of $x_{1}$, where the translation will be fixed later. Then the core of $t_{j}$ lies on the plane $P_{2 j \pi / 5}$; and the planes containing the cores of two consecutive tori in $\left\{T_{0}, t_{1}, t_{2}, t_{3}, t_{4}, T_{5}\right\}$ form an angle $2 \pi / 5$.

The numbers $A>B>1, a>b>\lambda>0$, and $a^{\prime}>b^{\prime}>\lambda^{\prime}>0$ are retained from the previous cases. To realize the plan, we need to choose $a^{\prime \prime}>b^{\prime \prime}>\lambda^{\prime \prime}>0$ 
satisfying $a^{\prime \prime} / A=b^{\prime \prime} / B=\lambda^{\prime \prime} / 1<\lambda$, so that the 4 new tori $t_{1}, t_{2}, t_{3}, t_{4}$ can be fitted lengthwise in the space vacated by $T_{2}, T_{3}$, and $T_{4}$ to form a necklace chain. The calculations leading to these choices are routine and tedious; we omit the details. This completes the proof.

\section{The Bing double and the Whitehead continuum revisited}

The construction of the space $\mathbb{R}^{3} / \mathrm{Bd}$ associated to the Bing double is illustrated and discussed in Daverman's book (see Example 1, pp. 62-63 in [6]) and in an article of Freedman and Skora [8]. See the original article [1] or [4] for a highly nontrivial shrinking procedure that leads to a homeomorphism $\mathbb{R}^{3} / \mathrm{Bd} \approx \mathbb{R}^{3}$.

We fix an initial package consisting of three tori $T, T_{1}$, and $T_{2}$ in $\mathbb{R}^{3}$ so that $T_{1}$ and $T_{2}$ are linked in $T$ but not in $\mathbb{R}^{3}$ as in Figure $9-1$ of [6], and we fix the homeomorphisms $\phi_{i}: T \rightarrow T_{i}$. Denote by $\mathcal{X}=\left(X_{k}\right)$ the defining sequence induced by the initial package as described in Section 4.2, by Bd the (cellular) decomposition, and by $\mathbb{R}^{3} / \mathrm{Bd}$ the decomposition space.

Semmes showed (Theorem 1.12c in [19]) that $\mathbb{R}^{3} / \mathrm{Bd}$ admits a metric $d$ so that the space $\left(\mathbb{R}^{3} / \mathrm{Bd}, d\right)$ is quasiconvex, Ahlfors 3-regular, and linearly locally contractible and it supports certain Sobolev and Poincaré inequalities that are crucial for analysis, but this space is not quasisymmetric to $\mathbb{R}^{3}$. Semmes's construction of the metric $d$ served as a model for the modular metrics defined in Section 7; it is easy to verify that $d$ is bilipschitz equivalent to a modular metric.

The nonexistence of a quasisymmetric homeomorphism $\left(\mathbb{R}^{3} / \mathrm{Bd}, d\right) \rightarrow \mathbb{R}^{3}$ is based on a lemma of Freedman and Skora on essential intersections (Lemma 2.4 in $[8])$.

We state their lemma in the following.

Lemma 17.1. Let $T_{1}$ and $T_{2}$ be solid tori embedded in $\mathbb{B}^{2} \times \mathbb{S}^{1}$ as in the Bing double construction. Let $(P, \partial P) \subset\left(\mathbb{B}^{2} \times \mathbb{S}^{1}, \partial \mathbb{B}^{2} \times \mathbb{S}^{1}\right)$ be an embedded connected planar surface representing the generator of the relative homology group $H_{2}\left(\mathbb{B}^{2} \times \mathbb{S}^{1}, \partial, \mathbb{Z}\right)$. Suppose $P$ and $T_{1} \cup T_{2}$ meet in transverse general position. Then for $i=1$ or 2 , $P \cap T_{i}$ must contain at least two surfaces which represent generators of $H_{2}\left(T_{i}, \partial, \mathbb{Z}\right)$.

Using the notion of circulation, Lemma 17.1 can be interpreted as follows.

Lemma 17.2. Let $\mathbb{R}^{3} / \mathrm{Bd}$ be the decomposition space associated to the Bing double $B d$ and let $\mathcal{X}=\left(X_{k}\right)$ be the defining sequence associated to the initial package $\left(T, T_{1}, T_{2}, \phi_{1}, \phi_{2}\right)$. Then

$$
\operatorname{circ}\left(X_{k}, T ; \alpha\right) \geq 2^{k}
$$

for every $k \geq 0$ and every meridian $\alpha$ on $T$.

The Freedman-Skora lemma yields that the defining sequence $\mathcal{X}$ of the Bing double has order of circulation at least 2 ; in fact the order of growth of $\mathcal{X}$ is exactly 2. Theorem 1.4 now follows from Theorem 13.1 . 
By the Freedman-Skora lemma, the concurrent pair $(\gamma(x), \omega(x))$ defined in Section 14 can be taken to be $(2,2)$ for every $x$ in $\pi_{\mathrm{Bd}}\left(\mathrm{Bd}_{\infty}\right)$. From Theorem 14.3, it follows that

1. any open subset of $\left(\mathbb{R}^{3} / \mathrm{Bd}, d_{\lambda}\right)$ which intersects $\pi_{\mathrm{Bd}}\left(\mathrm{Bd}_{\infty}\right)$ is not quasisymmetrically embeddable in $\mathbb{R}^{3}$ for any $0<\lambda<1$;

2. every point in $\pi_{\mathrm{Bd}}\left(\mathrm{Bd}_{\infty}\right)$ is $\lambda$-singular (of index 1 ) for $1 / 2<\lambda<1$.

We finish the proof of Theorem 1.5 using the second fact.

Proof of Theorem 1.5. Suppose there is a quasisymmetric homeomorphism $f$ : $\left(\mathbb{R}^{3} / \mathrm{Bd} \times \mathbb{R}, d_{\lambda, 1}\right) \rightarrow\left(\mathbb{R}^{3} / \mathrm{Bd} \times \mathbb{R}, d_{\lambda^{\prime}, 1}\right)$ for some $\lambda^{\prime} \in(1 / 2,1)$ and $\lambda \in\left(0, \lambda^{\prime}\right)$. Let $\mathcal{X}=\left(\mathrm{Bd}_{k}\right)$ be the standard defining sequence for the Bing double, and write $\mathrm{Bd}_{\infty}=\bigcap_{k} \mathrm{Bd}_{k}$. By Theorems 14.3 and 15.1,

$$
\pi_{\mathrm{Bd}}\left(\mathrm{Bd}_{\infty}\right)=\operatorname{sing}_{\lambda, 1}\left(\mathbb{R}^{3} / \mathrm{Bd}\right)=\operatorname{sing}_{\lambda^{\prime}, 1}\left(\mathbb{R}^{3} / \mathrm{Bd}\right) .
$$

Since $\pi_{\mathrm{Bd}}\left(\mathrm{Bd}_{\infty}\right)$ is a Cantor set, every point is an accumulation point. By Theorem 15.2, given $\ell>0$ there exists $k_{0}>0$ so that

$$
f\left(\pi_{\mathrm{Bd}}\left(\mathrm{Bd}_{k}\right) \times \mathbb{R}\right) \subset \pi_{\mathrm{Bd}}\left(\mathrm{Bd}_{k+\ell}\right) \times \mathbb{R}
$$

for all $k \geq k_{0}$. Since $\pi_{\mathrm{Bd}}\left(\mathrm{Bd}_{k}\right)$ has $2^{k}$ components and $\pi_{\mathrm{Bd}}\left(\mathrm{Bd}_{k+\ell}\right)$ has $2^{k+\ell}$ components, we conclude that there exists a component $T$ of $\mathrm{Bd}_{k+\ell}$ so that

$$
\pi_{\mathrm{Bd}}(T) \times \mathbb{R} \cap f\left(\pi_{\mathrm{Bd}}\left(\mathrm{Bd}_{\infty}\right) \times \mathbb{R}\right)=\emptyset .
$$

This contradicts (17.1).

We refer to [12] for the nonexistence of the quasisymmetric parametrization of $\mathbb{R}^{3} / \mathrm{Wh} \times \mathbb{R}^{m}$, where Wh is the Whitehead continuum. We merely note that the homological argument of the Freedman-Skora lemma was used in [12] to obtain a version of the intersection lemma and to show that the standard defining sequence for the Whitehead continuum has order of circulation at least 2. Since the order of growth of the Whitehead continuum is 1 , we can use Theorem 13.1 to recover the nonexistence of quasisymmetric parametrizations of $\left(\mathbb{R}^{3} / \mathrm{Wh} \times \mathbb{R}^{m}, d_{\lambda, m}\right)$. Indeed, as shown in $[12],\left(\mathbb{R}^{3} / \mathrm{Wh} \times \mathbb{R}^{m}, d_{\lambda, m}\right)$ is not quasisymmetric to $\mathbb{R}^{3+m}$ for $\lambda>2^{-(3+m) /(2 m)}$.

\section{Bing's dogbone}

The decomposition space $\mathbb{R}^{3} / \mathrm{Db}$ associated with Bing's dogbone [2] was the first known example of a decomposition space which is not homeomorphic to $\mathbb{R}^{3}$ but whose product with a line, $\left(\mathbb{R}^{3} / \mathrm{Db}\right) \times \mathbb{R}$, is homeomorphic to $\mathbb{R}^{4}$; see [3].

Bing's dogbone space $\mathbb{R}^{3} / \mathrm{Db}$ is constructed as follows. Let $A$ be a PL cubewith-2-handles standardly embedded in $\mathbb{R}^{3}$, and let $A_{1}, A_{2}, A_{3}$, and $A_{4}$ be four cubes-with-handles of genus 2 embedded in the interior of $A$ as illustrated in Figure 1, p. 486, of [2]. 
Let $\phi_{j}: U \rightarrow U_{j}$ be PL-homeomorphisms from a neighborhood $U$ of $A$ onto mutually disjoint neighborhoods $U_{i}, 1 \leq i \leq 4$, of $A_{i}$ satisfying $A_{i} \subset U_{i} \subset A \subset U$. The intersection

$$
\mathrm{Db}=\bigcap_{k=0}^{\infty} \bigcup_{\alpha \in S_{k}} \phi_{\alpha}(A)
$$

is called Bing's dogbone, where $\phi_{\alpha}=\phi_{\alpha_{1}} \circ \cdots \circ \phi_{\alpha_{k}}$ and $\alpha=\left(\alpha_{1}, \ldots, \alpha_{k}\right) \in$ $\{1,2,3,4\}^{k}$. The decomposition $\mathbb{R}^{3} / \mathrm{Db}$ is topologically different from $\mathbb{R}^{3}$ even though each nondegenerate component of $\mathrm{Db}$ is a tame arc [2]. On the other hand, $\left(\mathbb{R}^{3} / \mathrm{Db}\right) \times \mathbb{R}$ is $\mathbb{R}^{4}$.

The initial package $\left(A, A_{1}, \ldots, A_{4}, \phi_{1}, \ldots, \phi_{4}\right)$ yields a defining sequence $\mathcal{X}_{\mathrm{Db}}=$ $\left(X_{k}\right): X_{0}=A$ and

$$
X_{k+1}=\bigcup_{\alpha=1}^{4} \phi_{\alpha}\left(X_{k}\right)
$$

for $k \geq 0$. Hence $X_{k}=\bigcup_{\alpha \in \mathcal{S}_{k}} \phi_{\alpha}(A)$. The initial package induces a welding structure $\left(\mathcal{C}_{\mathrm{Db}}, \mathcal{A}_{\mathrm{Db}}, \mathcal{W}_{\mathrm{Db}}\right)$ on the defining sequence $\mathcal{X}_{\mathrm{Db}}$; in particular $\mathcal{C}$ consists of a single condenser $\left(A, \cup_{i=1}^{4} A_{i}\right)$. See Section 4.2 for details.

Theorem 18.1. Let $\left(\mathbb{R}^{3} / \mathrm{Db}, d_{\lambda}\right)$ be a Semmes space associated to the defining sequence $\mathcal{X}_{D b}$ and the welding structure $\left(\mathcal{C}_{\mathrm{Db}}, \mathcal{A}_{\mathrm{Db}}, \mathcal{W}_{\mathrm{Db}}\right)$. Suppose $m \geq 1$ and $2^{-(1+m) / m}<\lambda<2^{-2 / 3}$. Then $\left(\mathbb{R}^{3} / \mathrm{Db} \times \mathbb{R}^{m}, d_{\lambda, m}\right)$ is Ahlfors $(3+m)$-regular and linearly locally contractible, but it is not quasisymmetrically equivalent to $\mathbb{R}^{3+m}$.

The Ahlfors regularity follows from Proposition 7.8, since $\mathcal{X}$ has order of growth 4 . The linear local contractibility follow from Proposition 7.9, since every $A_{i}$ is contractible in $A$.

To show that $\left(\mathbb{R}^{3} / \mathrm{Db}\right) \times \mathbb{R}^{m}$ is not quasisymmetric to $\mathbb{R}^{3+m}$, we estimate the order of circulation of $\mathcal{X}$ in $A$ from below.

As in Figure 1 of [2], let $C_{1}$ and $C_{2}$ be two disjoint 3 -cells in $A$ so that the handles of $\cup A_{i}$ are sorted into two groups, and each group consists of four pairwise linked handles, one from each $A_{i}$, and is contained in one of the 3-cells $C_{1}$ or $C_{2}$. Then $C_{1} \cup C_{2} \cup A_{1} \cup A_{4}$ and $C_{1} \cup C_{2} \cup A_{2} \cup A_{3}$ is a pair of solid tori in $A$.

The arrangement of cubes-with-handles $\cup A_{i}$ is understood as follows. We fix essential 2-disks $D_{1}, D_{2}$, and $D_{3}$ in $A$ as in Figure 1 of [2]. These disks have the property that if $h: \mathbb{R}^{3} \rightarrow \mathbb{R}^{3}$ is a homeomorphism that is identity outside $A$ then

1. $h\left(A_{1}\right) \cup h\left(A_{4}\right)$ and $h\left(A_{2}\right) \cup h\left(A_{3}\right)$ intersect both $D_{1}$ and $D_{2}$,

2. $h\left(A_{1}\right) \cup h\left(A_{3}\right)$ and $h\left(A_{2}\right) \cup h\left(A_{4}\right)$ intersect both $D_{1}$ and $D_{3}$, and

3. $h\left(A_{1}\right) \cup h\left(A_{2}\right)$ and $h\left(A_{3}\right) \cup h\left(A_{4}\right)$ intersect both $D_{2}$ and $D_{3}$.

We use topological properties of the initial package to show the following estimate of Freedman-Skora type. This estimate implies that the order of circulation of $\mathcal{X}$ is at least 4 . This together with Theorem 13.1 proves Theorem 18.1.

Lemma 18.2. Let $\gamma$ be a meridian of $A$ that is isotopic to $\partial D_{1}$ on $\partial A$. Then

$$
\operatorname{circ}\left(X_{k}, \gamma, A\right) \geq 4^{k-1}
$$

for every $k \geq 1$. 
Proof. As a preliminary step, we define tori $T^{O}$ and $T^{X}$ as follows

$$
T^{O}=C_{1} \cup C_{2} \cup A_{1} \cup A_{4} \text { and } T^{X}=C_{1} \cup C_{2} \cup A_{2} \cup A_{3} .
$$

Then

$$
A_{\alpha 1} \cup A_{\alpha 4} \subset\left(T^{O}\right)_{\alpha} \subset A_{\alpha} \text { and } A_{\alpha 2} \cup A_{\alpha 3} \subset\left(T^{X}\right)_{\alpha} \subset A_{\alpha},
$$

where $A_{\alpha}=\phi_{\alpha} A,\left(T^{O}\right)_{\alpha}=\phi_{\alpha}\left(T^{O}\right)\left(T^{X}\right)_{\alpha}=\phi_{\alpha}\left(T^{X}\right)$, and $\alpha \in\{1,2,3,4\}^{k}$.

Note that tori $\left(T^{O}\right)_{1} \cup\left(T^{O}\right)_{4}$ are linked in $T^{O}$ the way that the two first stage tori are linked in the 0 -th stage torus as in the construction of the Bing double. Note also that the same can be said about the linking of $\left(T^{X}\right)_{1} \cup\left(T^{X}\right)_{4}$ in $T^{O}$, $\left(T^{O}\right)_{2} \cup\left(T^{O}\right)_{3}$ in $T^{X}$, and $\left(T^{X}\right)_{2} \cup\left(T^{X}\right)_{3}$ in $T^{X}$.

Therefore for every $\alpha \in\{1,2,3,4\}^{k}$, the tori $\left(T^{O}\right)_{\alpha 1} \cup\left(T^{O}\right)_{\alpha 4}$ are linked in $\left(T^{O}\right)_{\alpha}$ the way the first stage tori are linked in the 0th stage torus as in the Bing double, and the same can be said about the linking of $\left(T^{X}\right)_{\alpha 1} \cup\left(T^{X}\right)_{\alpha 4}$ in $\left(T^{O}\right)_{\alpha}$, $\left(T^{O}\right)_{\alpha 2} \cup\left(T^{O}\right)_{\alpha 3}$ in $\left(T^{X}\right)_{\alpha}$ and $\left(T^{X}\right)_{\alpha 2} \cup\left(T^{X}\right)_{\alpha 3}$ in $\left(T^{X}\right)_{\alpha}$.

This linking property has the following consequences.

(I) If $f:\left(\mathbb{B}^{2}, \partial \mathbb{B}^{2}\right) \rightarrow\left(A, \partial D_{1}\right)$ is map with the property $f\left(\partial \mathbb{B}^{2}\right)=\partial D_{1}$, then $f\left(\mathbb{B}^{2}\right)$ intersects both $T^{O}$ and $T^{X}$ virtually interior essentially. Indeed, let $Q$ be a 3-cell in $\mathbb{R}^{3}$ so that $Q \cap A \subset \partial A, Q \cap \partial D_{1}=\emptyset$, and so that $Q \cup A$ is a torus. We write $T=Q \cup A$. Since a core of $T^{O}$ is also a core of $T$, we have that $f\left(\mathbb{B}^{2}\right)$ intersects $T^{O}$ virtually interior essentially. The same argument applies also to $T^{X}$.

(II) Suppose $\Omega$ is a 2-manifold in $\mathbb{B}^{2}$ and $f:(\Omega, \partial \Omega) \rightarrow\left(T^{O}, \partial T^{O}\right)$ is a virtually interior essential map. Then by the standard argument of filling $T^{O}$ with 2 -disks, we have that $f$ has an virtually interior essential intersection with $A_{1} \cup A_{4}$; see e.g. the proof of the wildness of Antoine's necklace in Proposition 5, pp. 73-74 of [6]. The same can be said about $T^{X}$ and $A_{2} \cup A_{3}$.

The circulation estimate (18.1) follows from the claim below and the relation between the number of essential intersections and the circulation stated in Remark 9.8 .

Claim. Let $f:\left(\mathbb{B}^{2}, \partial \mathbb{B}^{2}\right) \rightarrow(A, \partial A)$ be an interior essential map so that $f\left(\partial \mathbb{B}^{2}\right)$ is isotopic to $\partial D_{1}$ on $\partial A$. Then $f\left(\mathbb{B}^{2}\right) \cap X_{k}$ has at least $4^{k}$ virtually interior essential components. It remains to verify the claim.

Let $\varsigma:\{1,2,3,4\} \rightarrow\{O, X\}$ be the map defined by $\varsigma(1)=\varsigma(4)=O$ and $\varsigma(2)=\varsigma(3)=X$, and let $\varsigma^{k}=\varsigma \times \cdots \times \varsigma:\{1,2,3,4\}^{k} \rightarrow\{O, X\}^{k}$ be the product map. Set $\mathcal{S}_{k}=\{1,2,3,4\}^{k}, \Sigma_{k}=\{O, X\}^{k}$, and $s_{k}(w)=\left(\varsigma^{k}\right)^{-1}(w)$. Note that, for $w=\left(w_{1}, \ldots, w_{k}\right) \in \Sigma_{k}$,

$$
s_{k}(w)=\left(\varsigma^{k}\right)^{-1}(w)=\left\{\left(\alpha_{1}, \ldots, \alpha_{k}\right) \in \mathcal{S}_{k}, \alpha_{j} \in \varsigma^{-1}\left(w_{j}\right) \text { for all } 1 \leq j \leq k\right\},
$$

so $\mathcal{S}_{k}=\cup_{w \in \Sigma_{k}} s_{k}(w)$ is a disjoint union.

For each $k \geq 1$, we sort the $4^{k}$ cubes-with-handles in $X_{k}$ into $2^{k}$ mutually disjoint groups as follows. If $k=1$, the two groups are $X_{1}(O)=\left\{A_{1}, A_{4}\right\}$ and $X_{1}(X)=\left\{A_{2}, A_{3}\right\}$. Suppose $k \geq 2$. Define, for $w \in \Sigma_{k}$,

$$
X_{k}(w)=\left\{A_{\alpha}: \alpha \in s_{k}(w)\right\},
$$


so $X_{k}=\cup_{w \in \Sigma_{k}} X_{k}(w)$ is a disjoint union of $2^{k}$ groups.

Fix a $w \in \Sigma_{k}$. We will focus on the $2^{k}$ cubes-with-handles in $X_{k}(w)$ and consider a finite defining sequence associated with this particular $w=\left(w_{1}, w_{2}, \ldots, w_{k}\right)$ as follows. Set

$$
Z_{0}=A, \quad Z_{1}=T^{w_{1}}, \quad \text { and } \quad Z_{j}=\cup_{\alpha \in s_{j-1}\left(w_{1}, w_{2}, \ldots, w_{j-1}\right)}\left(T^{w_{j}}\right)_{\alpha}
$$

for $2 \leq j \leq k$. Note that

$$
Z_{j+1} \cap\left(T^{w_{j}}\right)_{\alpha}=\left(T^{w_{j+1}}\right)_{\alpha i_{1}} \cup\left(T^{w_{j+1}}\right)_{\alpha i_{2}},
$$

where $\left\{i_{1}, i_{2}\right\}=\varsigma^{-1}\left(w_{j}\right)$, for every $\left(T^{w_{j}}\right)_{\alpha}$ in $Z_{j}$.

Let $f:\left(\mathbb{B}^{2}, \partial \mathbb{B}^{2}\right) \rightarrow(A, \partial A)$ be an interior essential map so that $f\left(\partial \mathbb{B}^{2}\right)$ is isotopic to $\partial D_{1}$ on $\partial A$. By applying a homotopy near $\partial A$, we may assume that $f\left(\mathbb{B}^{2}\right) \cap \partial A=\partial D_{1}$. Then, by $(\mathrm{I}), f\left(\mathbb{B}^{2}\right)$ intersects both $T^{O}$ and $T^{X}$ virtually interior essentially. In particular, $f\left(\mathbb{B}^{2}\right) \cap Z_{1}$ has at least one virtually interior essential component.

In view of the linking relation (of the Bing double type) between tori in consecutive generations, we may apply the lemma of Freedman and Skora (Lemma 17.1) iteratively to conclude that $f\left(\mathbb{B}^{2}\right) \cap Z_{k}$ has at least $2^{k-1}$ virtually interior essential components.

The tori in $Z_{k}$ are pairwise disjoint and each torus contains two cubes-withhandles in $X_{k}(w)$. It follows from (II) above that $f\left(\mathbb{B}^{2}\right) \cap X_{k}(w)$ has at least $2^{k-1}$ virtually interior essential components.

The claim follows by summing over $w \in \Sigma_{k}$. This completes the proof of the theorem.

\section{References}

[1] Bing, R.H.: A homeomorphism between the 3-sphere and the sum of two solid horned spheres. Ann. of Math. (2) 56 (1952), 354-362.

[2] Bing, R. H.: A decomposition of $E^{3}$ into points and tame arcs such that the decomposition space is topologically different from $E^{3}$. Ann. of Math. (2) 65 (1957), 484-500.

[3] Bing, R. H.: The cartesian product of a certain nonmanifold and a line is $E^{4}$. Ann. of Math. (2) 70 (1959), 399-412.

[4] Bing, R. H.: Shrinking without lengthening. Topology 27 (1988), no. 4, 487-493.

[5] Bonk, M. And Kleiner, B.: Quasisymmetric parametrizations of two-dimensional metric spheres. Invent. Math. 150 (2002), no. 1, 127-183.

[6] Daverman, R. J.: Decompositions of manifolds. Pure and Applied Mathematics 124, Academic Press, Orlando, FL, 1986.

[7] Edwards, R. D. And Miller, R. T.: Cell-like closed-0-dimensional decompositions of $R^{3}$ are $R^{4}$ factors. Trans. Amer. Math. Soc. 215 (1976), 191-203.

[8] Freedman, M. H. and Skora, R.: Strange actions of groups on spheres. J. Differential Geom. 25 (1987), no. 1, 75-98. 
[9] Heinonen, J. And Koskela, P.: Quasiconformal maps in metric spaces with controlled geometry. Acta Math. 181 (1998), no. 1, 1-61.

[10] Heinonen, J. and Rickman, S.: Quasiregular maps $\mathbf{S}^{3} \rightarrow \mathbf{S}^{3}$ with wild branch sets. Topology 37 (1998), no. 1, 1-24.

[11] Heinonen, J. And Semmes, S.: Thirty-three yes or no questions about mappings, measures, and metrics. Conform. Geom. Dyn. 1 (1997), 1-12 (electronic).

[12] Heinonen, J. And Wu, J.-M.: Quasisymmetric nonparametrization and spaces associated with the Whitehead continuum. Geom. Topol. 14 (2010), no. 2, 773-798.

[13] Hempel, J.: 3-Manifolds. Annals of Mathematics Studies 86, Princeton University Press, Princeton, NJ, 1976.

[14] Lambert, H. W. And Sher, R. B.: Point-like 0-dimensional decompositions of $S^{3}$. Pacific J. Math. 24 (1968), 511-518.

[15] Penrose, R., Whitehead, J. H. C. and Zeeman, E. C.: Imbedding of manifolds in euclidean space. Ann. of Math. (2) 73 (1961), 613-623.

[16] Rourke, C. P. and Sanderson, B. J.: Introduction to piecewise-linear topology. Reprint. Springer Study Edition. Springer-Verlag, Berlin-New York, 1982.

[17] Semmes, S.: Chord-arc surfaces with small constant. II. Good parameterizations. Adv. Math. 88 (1991), no 2, 170-199.

[18] Semmes, S.: Finding curves on general spaces through quantitative topology, with applications to Sobolev and Poincaré inequalities. Selecta Math. (N.S.) 2 (1996), no. 2, 155-295.

[19] Semmes, S.: Good metric spaces without good parameterizations. Rev. Mat. Iberoamericana 12 (1996), no. 1, 187-275.

[20] Sher, R. B. And Alford, W. R.: A note on 0-dimensional decompositions of $E^{3}$. Amer. Math. Monthly $\mathbf{7 5}$ (1968), 377-378.

[21] Tukia, P. And VÄıs̈̈lä, J.: Quasisymmetric embeddings of metric spaces. Ann. Acad. Sci. Fenn. Ser. A I Math. 5 (1980), no 1, 97-114.

[22] Tyson, J.: Quasiconformality and quasisymmetry in metric measure spaces. Ann. Acad. Sci. Fenn. Math. 23 (1998), no. 2, 525-548.

[23] Wildrick, K.: Quasisymmetric parametrizations of two-dimensional metric planes. Proc. Lond. Math. Soc. (3) 97 (2008), no. 3, 783-812.

Received August 6, 2012.

Pekka Pankka: Department of Mathematics and Statistics, PO Box 68, 00014 University of Helsinki, Finland; and Department of Mathematics and Statistics, PO Box 35 (MaD), 40014 University of Jyväskylä, Finland.

E-mail: pekka.pankka@jyu.fi

JANG-MeI Wu: Department of Mathematics, University of Illinois, 1409 West Green Street, Urbana, IL 61822, USA.

E-mail: wu@math.uiuc.edu

The first author was supported in part by the Academy of Finland projects 126836 and 256228. The second author was supported in part by the National Science Foundation grants DMS-0757732 and DMS-1001669. 